\title{
An Improved Sphere-Packing Bound for Finite-Length Codes over Symmetric Memoryless Channels
}

\author{
Gil Wiechman \\ Igal Sason \\ Department of Electrical Engineering \\ Technion - Israel Institute of Technology \\ Haifa 32000, Israel
}

\begin{abstract}
This paper derives an improved sphere-packing (ISP) bound for finite-length codes whose transmission takes place over symmetric memoryless channels, and which are decoded with an arbitrary list decoder. We first review classical results, i.e., the 1959 sphere-packing (SP59) bound of Shannon for the Gaussian channel, and the 1967 sphere-packing (SP67) bound of Shannon et al. for discrete memoryless channels. An improvement on the SP67 bound by Valembois and Fossorier is also discussed. These concepts are used for the derivation of a new lower bound on the error probability of list decoding (referred to as the ISP bound) which is uniformly tighter than the SP67 bound and its improved version. The ISP bound is applicable to symmetric memoryless channels, and some of its applications are exemplified. Its tightness under ML decoding is studied by comparing the ISP bound to previously reported upper and lower bounds on the ML decoding error probability, and also to computer simulations of iteratively decoded turbo-like codes. This paper also presents a technique which performs the entire calculation of the SP59 bound in the logarithmic domain, thus facilitating the exact calculation of this bound for moderate to large block lengths without the need for the asymptotic approximations provided by Shannon.
\end{abstract}

\section{Keywords}

Block codes, error exponent, list decoding, sphere-packing bound, turbo-like codes.

This paper was published in the IEEE Trans. on Information Theory, vol. 54, no. 5, pp. 1962-1990, May 2008. This post-print version is a result of some more editing, without any change in the content of the paper.

This work was presented in part at the 44th Annual Allerton Conference on Communication, Control and Computing, Monticello, Illinois, USA, September 2006; it was also presented at the 2006 IEEE 24th Convention of Electrical and Electronics Engineers in Israel, Eilat, Israel, November 2006.

This research work was supported by the Israel Science Foundation (grant no. 1070/07). 


\section{INTRODUCTION}

The theoretical study of the fundamental performance limitations of long block codes was initiated by Shannon. During the fifties and sixties, this research work attracted Shannon and his colleagues at MIT and Bell Labs (see, e.g., the collected papers of Shannon [27] and the book of Gallager [12]). An overview of these classical results and their impact was addressed by Berlekamp [2].

The 1959 sphere-packing (SP59) bound of Shannon [25] serves for the evaluation of the performance limits of block codes whose transmission takes place over an AWGN channel. This lower bound on the decoding error probability is expressed in terms of the block length and rate of the code; however, it does not take into account the modulation used, but only assumes that the signals are of equal energy. It is often used as a reference for quantifying the sub-optimality of error-correcting codes under some practical decoding algorithms.

The 1967 sphere-packing (SP67) bound, derived by Shannon, Gallager and Berlekamp [26], provides a lower bound on the decoding error probability of block codes as a function of their block length and code rate, and it holds for general discrete memoryless channels. Like the random-coding bound (RCB) of Gallager [11], the SP67 bound decays to zero exponentially with the block length for all rates below the channel capacity. Further, the error exponent of the SP67 bound is known to be tight at the portion of the rate region between the critical rate $\left(R_{\mathrm{c}}\right)$ and the channel capacity; for all the rates in this range, the error exponents of the SP67 and the random-coding bounds coincide (see [26, Part 1]).

The introduction of turbo-like codes, which closely approach the Shannon capacity limit with moderate block lengths and a feasible decoding complexity, stirred up new interest in studying the limits of code performance as a function of the block length (see, e.g., [9], [15], [16], [18], [24], [30], [36], [38]). In a recent paper [3], Costello and Forney survey the evolution of channel coding techniques, and also address the significant contributions of error-correcting codes in improving the tradeoff between performance, block length (delay) and complexity for practical applications.

In spite of the exponential decay of the SP67 bound in terms of the block length at all rates below the channel capacity, this bound appears to be loose for codes of small to moderate block lengths. The weakness of this bound is due to the original focus in [26] on asymptotic analysis. In [36], Valembois and Fossorier revisited the SP67 bound in order to improve its tightness for finite-length block codes (especially, for codes of short to moderate block lengths), and also extended its validity to memoryless continuous-output channels (e.g., the binary-input AWGN channel). The remarkable improvement of their bound over the classical SP67 bound was exemplified in [36]. Moreover, the extension of the bound in [36] to memoryless continuousoutput channels provides an alternative to the SP59 bound for the AWGN channel [25].

This paper is focused on the study of the fundamental performance limitations of finitelength error-correcting codes and the tradeoff between their performance and block length when the transmission takes place over an arbitrary symmetric memoryless channel. This study is facilitated by theoretical bounds, and it is also compared to the performance of modern coding techniques under sub-optimal and practical decoding algorithms. In this work, we derive an improved sphere-packing bound (referred to as the 'ISP bound') which improves the bounding techniques in [26] and [36], especially for codes of short to moderate block lengths; this new bound is valid for all symmetric memoryless channels.

The structure of this paper is as follows: Section II reviews the concepts which were used for the derivation of the SP67 bound [26, Part 1] and its improved version in [36]. In Section III, we derive the ISP bound which improves the bound in [36] for symmetric memoryless channels 
where the derivation of the ISP bound relies on concepts and notation presented in Section II. Section IV starts by reviewing the SP59 bound of Shannon [25], and presenting an algorithm used in [36] for a numerical calculation this bound. The numerical instability of this algorithm for codes of moderate to large block lengths motivates the derivation of an alternative algorithm in Section IV which facilitates the exact calculation of the SP59 bound, irrespectively of the block length. Section V provides numerical results which serve to compare the ISP bound to previously reported sphere-packing bounds. The tightness of the ISP bound is exemplified in Section V for various communication channels. Additionally, sphere-packing bounds are applied in Section $\mathrm{V}$ to study the tradeoff between the performance and the required block length of error-correcting codes. We conclude our discussion in Section VI. Some technical details are relegated to the appendices.

\section{THE 1967 SPHERE-PACKING BOUND AND IMPROVEMENTS}

In the following, we present the SP67 bound and its improvement in [36], followed by an outline of their derivation. Classical sphere-packing bounds are reviewed in [24, Chapter 5]. This section serves as a preparatory step towards the derivation of an improved sphere-packing bound in the next section.

\section{A. The 1967 Sphere-Packing Bound}

Let us consider a block code $\mathcal{C}$ which consists of $M$ codewords each of length $N$, and denote its codewords by $\mathbf{x}_{1}, \ldots, \mathbf{x}_{M}$. Assume that $\mathcal{C}$ is transmitted over a discrete memoryless channel (DMC) and is decoded by a list decoder; for each received sequence $\mathbf{y}$, the decoder outputs a list of at most $L$ integers from the set $\{1,2, \ldots, M\}$ which correspond to the indices of the codewords. A list decoding error is declared if the index of the transmitted codeword does not appear in the list. Originally introduced by Elias [10] and Wozencraft [39], list decoding signifies an important class of decoding algorithms. During the last decade, there has been a significant breakthrough in the construction of efficient list-decoding algorithms for error-correcting codes (see, e.g., [13], [22, Chapter 9] and references therein).

A lower bound on the decoding error probability of an arbitrary block code with $M$ codewords of length $N$ is derived in [26]. This bound applies to an arbitrary list decoder where the size of the list is limited to $L$. The particular case where $L=1$ clearly provides a lower bound on the error probability under maximum-likelihood (ML) decoding.

Let $\mathcal{Y}_{m}$ designate the set of output sequences $\mathbf{y}$ for which message $m$ is on the decoding list, and define $P_{m}(\mathbf{y}) \triangleq \operatorname{Pr}\left(\mathbf{y} \mid \mathbf{x}_{m}\right)$. The conditional error probability under list decoding when message $m$ is sent over the channel is given by

$$
P_{\mathrm{e}, m}=\sum_{y \in \mathcal{Y}_{m}^{\mathrm{c}}} P_{m}(\mathbf{y})
$$

where the superscript ' $c$ ' stands for the complementary set. For the block code and list decoder under consideration, let $P_{\mathrm{e}, \max }$ designate the maximal value of $P_{\mathrm{e}, m}$ where $m \in\{1,2, \ldots, M\}$. Assuming that all the codewords are equally likely to be transmitted, the average decoding error probability is given by

$$
P_{\mathrm{e}}=\frac{1}{M} \sum_{m=1}^{M} P_{\mathrm{e}, m} .
$$

Referring to a list decoder of size at most $L$, the code rate is defined as $R \triangleq \frac{\ln \left(\frac{M}{L}\right)}{N}$ nats per channel use. 
The derivation of the SP67 bound [26, Part 1] is divided into three main steps. The first step refers to the derivation of upper and lower bounds on the error probability of a code consisting of two codewords only. These bounds are given by the following theorem:

Theorem 2.1 (Upper and Lower Bounds on the Pairwise Error Probability): [26, Theorem 5]. Let $P_{1}$ and $P_{2}$ be probability assignments defined over a discrete set of sequences, let $\mathcal{Y}_{1}$ and $\mathcal{Y}_{2}=\mathcal{Y}_{1}^{\mathrm{c}}$ be (disjoint) decision regions for these sequences, let $P_{\mathrm{e}, 1}$ and $P_{\mathrm{e}, 2}$ be given by (1), and assume that $P_{1}(\mathbf{y}) P_{2}(\mathbf{y}) \neq 0$ for at least one sequence $\mathbf{y}$. Then, for all $s \in(0,1)$

$$
P_{\mathrm{e}, 1}>\frac{1}{4} \exp \left(\mu(s)-s \mu^{\prime}(s)-s \sqrt{2 \mu^{\prime \prime}(s)}\right)
$$

or

$$
P_{\mathrm{e}, 2}>\frac{1}{4} \exp \left(\mu(s)+(1-s) \mu^{\prime}(s)-(1-s) \sqrt{2 \mu^{\prime \prime}(s)}\right)
$$

where

$$
\mu(s) \triangleq \ln \left(\sum_{\mathbf{y}} P_{1}(\mathbf{y})^{1-s} P_{2}(\mathbf{y})^{s}\right), \quad 0<s<1 .
$$

Furthermore, for an appropriate choice of the decision regions $\mathcal{Y}_{1}$ and $\mathcal{Y}_{2}$, the following upper bounds hold:

$$
P_{\mathrm{e}, 1} \leq \exp \left(\mu(s)-s \mu^{\prime}(s)\right)
$$

and

$$
P_{\mathrm{e}, 2} \leq \exp \left(\mu(s)+(1-s) \mu^{\prime}(s)\right) .
$$

The function $\mu$ is non-positive and convex over the interval $(0,1)$. The convexity of $\mu$ is strict unless $\frac{P_{1}(\mathbf{y})}{P_{2}(\mathbf{y})}$ is constant over all the sequences $\mathbf{y}$ for which $P_{1}(\mathbf{y}) P_{2}(\mathbf{y}) \neq 0$. Moreover, the function $\mu$ is strictly negative over the interval $(0,1)$ unless $P_{1}(\mathbf{y})=P_{2}(\mathbf{y})$ for all $\mathbf{y}$.

In the following, we present an outline of the proof of Theorem 2.1 which serves to emphasize the parallelism between Theorem 2.1 and the first part of the derivation of the ISP bound in Section III. A detailed proof of this theorem is given in [26, Section III].

Proof: Let us define the log-likelihood ratio (LLR) as

$$
D(\mathbf{y}) \triangleq \ln \left(\frac{P_{2}(\mathbf{y})}{P_{1}(\mathbf{y})}\right)
$$

and the probability distribution

$$
Q_{s}(\mathbf{y}) \triangleq \frac{P_{1}(\mathbf{y})^{1-s} P_{2}(\mathbf{y})^{s}}{\sum_{\mathbf{y}^{\prime}} P_{1}\left(\mathbf{y}^{\prime}\right)^{1-s} P_{2}\left(\mathbf{y}^{\prime}\right)^{s}}, \quad 0<s<1 .
$$

It is simple to show that for all $0<s<1$, the first and second derivatives of $\mu$ in (5) are equal to the statistical expectation and variance of the LLR, respectively, taken with respect to the probability distribution $Q_{s}$ in (9). This gives the following equalities:

$$
\begin{aligned}
\mu^{\prime}(s) & =\mathbb{E}_{Q_{s}}(D(\mathbf{y})) \\
\mu^{\prime \prime}(s) & =\operatorname{Var}_{Q_{s}}(D(\mathbf{y})) .
\end{aligned}
$$

Also, as can be readily verified from (5), (8) and (9)

$$
\begin{aligned}
& P_{1}(\mathbf{y})=\exp (\mu(s)-s D(\mathbf{y})) Q_{s}(\mathbf{y}) \\
& P_{2}(\mathbf{y})=\exp (\mu(s)+(1-s) D(\mathbf{y})) Q_{s}(\mathbf{y}) .
\end{aligned}
$$


For $0<s<1$, the equalities in (10) and (11) motivate the definition of a set of typical sequences with respect to the probability distribution $Q_{s}$ as follows:

$$
\mathcal{Y}_{s} \triangleq\left\{\mathbf{y} \in \mathcal{Y}:\left|D(\mathbf{y})-\mu^{\prime}(s)\right| \leq \sqrt{2 \mu^{\prime \prime}(s)}\right\} \text {. }
$$

For any choice of a decision region $\mathcal{Y}_{1}$, the conditional error probability given that the first message was transmitted satisfies

$$
\begin{aligned}
P_{\mathrm{e}, 1} & =\sum_{\mathbf{y} \in \mathcal{Y}_{1}^{\mathrm{c}}} P_{1}(\mathbf{y}) \\
& \geq \sum_{\mathbf{y} \in \mathcal{Y}_{1}^{\mathrm{c}} \cap \mathcal{Y}_{s}} P_{1}(\mathbf{y}) \\
& \stackrel{(a)}{=} \sum_{\mathbf{y} \in \mathcal{Y}_{1}^{\mathrm{c}} \cap \mathcal{Y}_{s}} \exp (\mu(s)-s D(\mathbf{y})) Q_{s}(\mathbf{y}) \\
& \stackrel{(b)}{\geq} \exp \left(\mu(s)-s \mu^{\prime}(s)-s \sqrt{2 \mu^{\prime \prime}(s)}\right) \sum_{\mathbf{y} \in \mathcal{Y}_{1}^{\mathrm{c}} \cap \mathcal{Y}_{s}} Q_{s}(\mathbf{y})
\end{aligned}
$$

where $(a)$ follows from (12) and $(b)$ relies on the definition of $\mathcal{Y}_{s}$ in (14). Using similar arguments and relying on (13), we also get that

$$
P_{\mathrm{e}, 2} \geq \exp \left(\mu(s)+(1-s) \mu^{\prime}(s)-(1-s) \sqrt{2 \mu^{\prime \prime}(s)}\right) \sum_{\mathbf{y} \in \mathcal{Y}_{2}^{c} \cap \mathcal{Y}_{s}} Q_{s}(\mathbf{y}) .
$$

Since $\mathcal{Y}_{1}$ and $\mathcal{Y}_{2}$ form a partition of the observation space, we have that

$$
\sum_{\mathbf{y} \in \mathcal{Y}_{1}^{\mathrm{c}} \cap \mathcal{Y}_{s}} Q_{s}(\mathbf{y})+\sum_{\mathbf{y} \in \mathcal{Y}_{2}^{\mathrm{c}} \cap \mathcal{Y}_{s}} Q_{s}(\mathbf{y})=\sum_{\mathbf{y} \in \mathcal{Y}_{s}} Q_{s}(\mathbf{y})>\frac{1}{2}
$$

where (17) relies on (10), (11), (14), and Chebychev's inequality. Hence, at least one of the two sums in the left side of (17) must be greater than $\frac{1}{4}$; in view of (15) and (16), it follows that at least one of the inequalities in (3) and (4) hold.

The upper bounds in (6) and (7) follow, respectively, from (12) and (13) with

$$
\begin{aligned}
& \mathcal{Y}_{1} \triangleq\left\{\mathbf{y} \in \mathcal{Y}: D(\mathbf{y})<\mu^{\prime}(s)\right\} \\
& \mathcal{Y}_{2}=\mathcal{Y}_{1}^{\mathrm{c}} .
\end{aligned}
$$

The initial motivation of Theorem 2.1 is the calculation of lower bounds on the error probability of a two-word code. Note that this theorem is valid for any pair of probability assignments $P_{1}$ and $P_{2}$ and decision regions $\mathcal{Y}_{1}$ and $\mathcal{Y}_{2}$ which form a partition of the observation space.

In the continuation of the derivation of the SP67 bound in [26], this theorem is used in order to control the size of a decision region of a particular codeword without directly referring to the other codewords. To this end, an arbitrary probability tilting measure $f_{N}$ is introduced in [26] over all $\mathrm{N}$-length sequences of channel outputs, requiring that it is factorized in the form

$$
f_{N}(\mathbf{y})=\prod_{n=1}^{N} f\left(y_{n}\right)
$$

for an arbitrary output sequence $\mathbf{y}=\left(y_{1}, \ldots, y_{N}\right)$. The size of the set $\mathcal{Y}_{m}$ is defined as

$$
F\left(\mathcal{Y}_{m}\right) \triangleq \sum_{\mathbf{y} \in \mathcal{Y}_{m}} f_{N}(\mathbf{y})
$$


Next, [26] relies on Theorem 2.1 in order to relate the conditional error probability $P_{\mathrm{e}, m}$ and $F\left(\mathcal{Y}_{m}\right)$ for fixed composition codes; this is done by associating $\operatorname{Pr}\left(\cdot \mid \mathbf{x}_{m}\right)$ and $f_{N}$ with $P_{1}$ and $P_{2}$, respectively. Theorem 2.1 is applied to derive a parametric lower bound on the size of the decision region $\mathcal{Y}_{m}$ or on the conditional error probability $P_{\mathrm{e}, m}$. Due to the fact that the list size is limited to $L$, then

$$
\sum_{m=1}^{M} F\left(\mathcal{Y}_{m}\right)=\sum_{m=1}^{M} \sum_{\mathbf{y} \in \mathcal{Y}_{m}} f_{N}(\mathbf{y}) \leq L
$$

since for every sequence $\mathbf{y}$, the relation $\mathbf{y} \in \mathcal{Y}_{m}$ holds for at most $L$ indices $m \in\{1, \ldots, M\}$, and $\sum_{\mathbf{y}} f_{N}(\mathbf{y})=1$. Therefore, there exists an index $m$ such that $F\left(\mathcal{Y}_{m}\right) \leq \frac{L}{M}$ and for this unknown value of $m$, one can upper bound the conditional error probability $P_{\mathrm{e}, m}$ by

$$
P_{\mathrm{e}, \max } \triangleq \max _{m \in\{1, \ldots, M\}} P_{\mathrm{e}, m} .
$$

Using Theorem 2.1 with the above setting for the probability assignments $P_{1}$ and $P_{2}$, then $P_{\mathrm{e}, 1}$ and $P_{\mathrm{e}, 2}$ in the left side of (3) and (4) are respectively replaced by $P_{\mathrm{e}, m}$ and $F\left(\mathcal{Y}_{m}\right)$. For the above unknown value of $m$, whose existence is assured to be in the set $\{1, \ldots, M\}$, one can replace $P_{\mathrm{e}, m}$ and $F\left(\mathcal{Y}_{m}\right)$ in the left side of (3) and (4) by their upper bounds $P_{\mathrm{e}, \max }$ and $\frac{L}{M}$, respectively. This provides a lower bound on $P_{\mathrm{e}, \max }$ as long as the inequality which follows from the replacement of $F\left(\mathcal{Y}_{m}\right)$ by its upper bound $\left(\frac{L}{M}\right)$ in the left side of (4) does not hold. Next, the probability assignment $f \triangleq f_{s}$ is optimized in [26], so as to get the tightest (i.e., maximal) lower bound on $P_{\mathrm{e}, \max }$ within this form while considering a code whose composition minimizes the bound (so that the bound holds for all fixed composition codes). A solution of this min-max problem, as provided in [26, Eqs. (4.18)-(4.20)], leads to the following theorem which gives a lower bound on the maximal decoding error probability of an arbitrary fixed composition block code (for a more detailed review of these concepts, see [24, Section 5.3]).

Theorem 2.2 (Lower Bound on the Maximal Error Probability of Fixed Composition Codes): [26, Theorem 6]. Let $\mathcal{C}$ be a fixed composition block code of $M$ codewords and length $N$. Assume that the transmission of $\mathcal{C}$ takes place over a DMC, and let $P(j \mid k)$ be the set of transition probabilities characterizing this channel (where $j \in\{0, \ldots, J-1\}$ and $k \in\{0, \ldots, K-1\}$ designate the channel output and input, respectively). For an arbitrary list decoder where the size of the list is limited to $L$, the maximal error probability $\left(P_{\mathrm{e}, \max }\right)$ satisfies

$$
P_{\mathrm{e}, \max } \geq \exp \left[-N\left(E_{\mathrm{sp}}\left(R-\frac{\ln 4}{N}-\varepsilon\right)+\sqrt{\frac{8}{N}} \ln \left(\frac{e}{\sqrt{P_{\min }}}\right)+\frac{\ln 4}{N}\right)\right]
$$

where $R \triangleq \frac{\ln \left(\frac{M}{L}\right)}{N}$ is the rate of the code, $P_{\min }$ designates the smallest non-zero transition probability of the DMC, the parameter $\varepsilon$ is an arbitrarily small positive number, and the function $E_{\text {sp }}$ is given by

$$
\begin{aligned}
& E_{\mathrm{sp}}(R) \triangleq \sup _{\rho \geq 0}\left(E_{0}(\rho)-\rho R\right) \\
& E_{0}(\rho) \triangleq \max _{\mathbf{q}} E_{0}(\rho, \mathbf{q}) \\
& E_{0}(\rho, \mathbf{q}) \triangleq-\ln \left(\sum_{j=0}^{J-1}\left[\sum_{k=0}^{K-1} q_{k} P(j \mid k)^{\frac{1}{1+\rho}}\right]^{1+\rho}\right) .
\end{aligned}
$$

The maximum in the right side of (25) is taken over all probability vectors $\mathbf{q}=\left(q_{0}, \ldots, q_{K-1}\right)$, i.e., over all $\mathbf{q}$ with $K$ non-negative components summing to 1 . 
The reason for considering fixed composition codes in [26] is that, in general, the optimal probability distribution $f_{s}$ may depend on the composition of the codewords through the choice of the parameter $s$ in $(0,1)$ (see [26, p. 96]).

The next step in the derivation of the SP67 bound is the application of Theorem 2.2 to obtain a lower bound on the maximal decoding error probability of an arbitrary block code. This is performed by lower bounding the maximal decoding error probability of a block code by the maximal error probability of its largest fixed composition subcode. Since the number of possible compositions is polynomial in the block length, one can lower bound the rate of the largest fixed composition subcode by $R-O\left(\frac{\ln N}{N}\right)$ where $R$ is the rate of the original code. Clearly, the rate loss caused by considering this subcode vanishes when the block length tends to infinity; however, it loosens the bound for codes of short to moderate block lengths. Finally, the bound on the maximal block error probability is transformed into a bound on the average block error probability by considering an expurgated code which contains half of the codewords of the original code with the lowest conditional error probability. This finally leads to the SP67 bound in [26, Part 1].

Theorem 2.3 (The 1967 Sphere-Packing Bound for Discrete Memoryless Channels): [26, Theorem 2]. Let $\mathcal{C}$ be an arbitrary block code whose transmission takes place over a DMC. Assume that the DMC is specified by the set of transition probabilities $P(j \mid k)$ where $k \in\{0, \ldots, K-1\}$ and $j \in\{0, \ldots, J-1\}$ designate the channel input and output alphabets, respectively. Assume that the code $\mathcal{C}$ forms a set of $M$ codewords of length $N$ (i.e., each codeword is a sequence of $N$ letters from the input alphabet), and consider an arbitrary list decoder where the size of the list is limited to $L$. Then, the average decoding error probability of the code $\mathcal{C}$ satisfies

$$
P_{\mathrm{e}}(N, M, L) \geq \exp \left\{-N\left[E_{\mathrm{sp}}\left(R-O_{1}\left(\frac{\ln N}{N}\right)\right)+O_{2}\left(\frac{1}{\sqrt{N}}\right)\right]\right\}
$$

where $R \triangleq \frac{\ln \left(\frac{M}{L}\right)}{N}$, and the error exponent $E_{\mathrm{sp}}(R)$ is introduced in (24). The terms

$$
\begin{aligned}
O_{1}\left(\frac{\ln N}{N}\right) & =\frac{\ln 8}{N}+\frac{K \ln N}{N}, \\
O_{2}\left(\frac{1}{\sqrt{N}}\right) & =\sqrt{\frac{8}{N}} \ln \left(\frac{e}{\sqrt{P_{\min }}}\right)+\frac{\ln 8}{N}
\end{aligned}
$$

scale asymptotically like $\frac{\ln N}{N}$ and $\frac{1}{\sqrt{N}}$, respectively, and $P_{\min }$ denotes the smallest non-zero transition probability of the DMC.

\section{B. Recent Improvements on the 1967 Sphere-Packing Bound}

The derivation of the SP67 bound has been revisited in [36], focusing on finite-length block codes. Four modifications of the derivation in [26] have been performed in [36] for improving the pre-exponent of the SP67 bound. In contrast to the SP67 bound, which only holds for DMCs, the improved bound in [36] also holds for memoryless channels with discrete input and continuous output alphabets. The improvements suggested in [36] are outlined in this section, and the resulting bound is introduced.

The first modification suggested in [36] is the addition of a free parameter in the derivation of the lower bound on the decoding error probability of two-word codes; this free parameter is used in conjunction with Chebychev's inequality, and it is optimized in order to tighten the lower bounds on $P_{\mathrm{e}, 1}$ and $P_{\mathrm{e}, 2}$ in Theorem 2.1 (see (3), (4)).

A second improvement presented in [36] is related to the inequality $s \sqrt{\mu^{\prime \prime}(s)} \leq \ln \left(\frac{e}{\sqrt{P_{\min }}}\right)$ which was applied to simplify the final form of the bound in Theorem 2.3 (see [26, Part 1]). This 
bound on the second derivative of $\mu$ results in no asymptotic loss, but it loosens the lower bound on the decoding error probability for finite-length codes (especially, for a short blocklength $N$ ); using the exact value of $\mu^{\prime \prime}$ instead, improves the tightness of the resulting bound in [36]. This modification also makes the bound suitable for memoryless channels with a continuous output alphabet, as it is no longer required that $P_{\min }>0$. It should be noted that this causes a small discrepancy in the derivation of the bound; the derivation of a lower bound on the block error probability which is uniform over all fixed composition codes relies on finding the composition which minimizes the lower bound. The optimal composition is given in [26, Eqs. (4.18), (4.19)] for the case where the upper bound on $\mu^{\prime \prime}$ is applied. In [36], the same composition is used without checking whether it is still the composition which minimizes the lower bound. However, as we shall see in the next section, for the class of symmetric memoryless channels, the value of the bound is independent of the code composition; therefore, the bound in [36, Theorem 7] (referred to as the 'VF bound') stays valid. This class of channels includes all memoryless binary-input output-symmetric (MBIOS) channels.

A third improvement in [36] refers to the particular selection of the value of $\rho \geq 0$ which leads to the derivation of Theorem 2.3. In [26], $\rho$ is set to be the value $\tilde{\rho}$ which maximizes the error exponent of the SP67 bound (i.e., the upper bound on the error exponent). This choice emphasizes the similarity between the error exponents of the SP67 bound and the RCB, hence proving that the error exponent of the SP67 bound is tight for all rates above the critical rate of the channel. In order to tighten the bound for finite-length block codes, [36] sets the value of $\rho$ to its optimal value $\rho^{*}$. For rates above the critical rate of the channel, the tightness of the error exponent of the SP67 bound implies that $\tilde{\rho}$ tends to $\rho^{*}$ as the block length tends to infinity. However, for codes of finite block length, this simple observation tightens the bound with almost no penalty in the computational complexity of the resulting bound.

The fourth observation made in [36] refers to the final stage in the derivation of the SP67 bound. In order to get a lower bound on the maximal decoding error probability of an arbitrary block code, the derivation in [26] considers the maximal decoding error probability of a fixed composition subcode of the original code. In [26], a simple lower bound on the size of the largest fixed composition subcode is given; namely, the size of the largest fixed composition subcode is not less than the size of the entire code divided by the number of possible compositions. Since the number of possible compositions is equal to the number of possible ways to divide $N$ symbols into $K$ types, this value is given by $\left(\begin{array}{c}N+K-1 \\ K-1\end{array}\right)$. To simplify the final expression of the SP67 bound, [26] relies on the inequality $\left(\begin{array}{c}N+K-1 \\ K-1\end{array}\right) \leq N^{K}$ which provides a simple upper bound on the number of compositions. Since this expression is polynomial is the block length $N$, there is no asymptotic loss to the error exponent. However, by using the exact expression for the number of possible compositions, the bound in [36] is tightened for codes of short to moderate block lengths.

These four modifications in the derivation of the SP67 bound in [26] are used in [36] to obtain an improved lower bound on the decoding error probability of block codes transmitted over memoryless channels with finite input alphabets. As mentioned above, these modifications also extend the validity of the new bound to memoryless channels with discrete input and continuous output. However, the requirement of a finite input alphabet still remains, as it is required to apply the bound to arbitrary block codes, and not only to fixed composition codes. Under the assumptions and notation used in Theorem 2.3, the VF bound [36] is introduced in the following theorem while keeping the notation of Theorem 2.3 [26, Theorem 2]:

Theorem 2.4 (Improvement on the Sphere-Packing Bound for Discrete Memoryless Channels): 
[36, Theorem 7]. The average decoding error probability satisfies

$$
P_{\mathrm{e}}(N, M, L) \geq \exp \left(-N E_{\mathrm{VF}}(R, N)\right)
$$

where

$$
E_{\mathrm{VF}}(R, N) \triangleq \inf _{x>\frac{\sqrt{2}}{2}}\left\{E_{0}\left(\rho_{x}\right)-\rho_{x}\left(R-O_{1}\left(\frac{\ln N}{N}, x\right)\right)+O_{2}\left(\frac{1}{\sqrt{N}}, x, \rho_{x}\right)\right\}
$$

and

$$
\begin{aligned}
& O_{1}\left(\frac{\ln N}{N}, x\right) \triangleq \frac{\ln 8}{N}+\frac{\ln \left(\begin{array}{c}
N+K-1 \\
K-1
\end{array}\right)}{N}-\frac{\ln \left(2-\frac{1}{x^{2}}\right)}{N} \\
& O_{2}\left(\frac{1}{\sqrt{N}}, x, \rho\right) \triangleq x \sqrt{\frac{8}{N} \sum_{k=0}^{K-1} q_{k, \rho} \nu_{k}^{(2)}(\rho)+\frac{\ln 8}{N}-\frac{\ln \left(2-\frac{1}{x^{2}}\right)}{N}} \\
& \nu_{k}^{(1)}(\rho) \triangleq \frac{\sum_{j=0}^{J-1} \beta_{j, k, \rho} \ln \frac{\beta_{j, k, \rho}}{P(j \mid k)}}{\sum_{j=0}^{J-1} \beta_{j, k, \rho}} \\
& \sum_{j=0}^{J-1} \beta_{j, k, \rho} \ln ^{2} \frac{\beta_{j, k, \rho}}{P(j \mid k)} \\
& \nu_{k}^{(2)}(\rho) \triangleq \frac{\sum_{j=0}^{J-1} \beta_{j, k, \rho}}{\beta_{j, k, \rho} \triangleq P(j \mid k)^{\frac{1}{1+\rho}}\left(\sum_{k}^{(1)}(\rho)\right]^{2}}\left(\sum_{k^{\prime}=0}^{K-1} q_{k^{\prime}, \rho} P\left(j \mid k^{\prime}\right)^{\frac{1}{1+\rho}}\right)^{\rho}
\end{aligned}
$$

where $\mathbf{q}_{\rho} \triangleq\left(q_{1, \rho}, \ldots, q_{K, \rho}\right)$ designates the input distribution which maximizes $E_{0}(\rho, \mathbf{q})$ in (25), and the parameter $\rho=\rho_{x}$ is determined by solving the equation

$$
R-O_{1}\left(\frac{\ln N}{N}, x\right)=-\frac{1}{\rho} \sum_{k=0}^{K-1} q_{k, \rho} \nu_{k}^{(1)}(\rho)+\frac{x}{\rho} \sqrt{\frac{2}{N} \sum_{k=0}^{K-1} q_{k, \rho} \nu_{k}^{(2)}(\rho) .}
$$

For a more detailed review of the improvements suggested in [36], the reader is referred to [24, Section 5.4].

Remark 2.1: [36] ignores the rate loss which follows from the expurgation of the code by a removal of half of the codewords with the largest error probability. Consequently, the term $\frac{\ln 4}{N}$ in $O_{1}\left(\frac{\ln N}{N}, x\right)$ of [36, Theorem 7] needs to be replaced by $\frac{\ln 8}{N}$ (see the right side of (31)). 


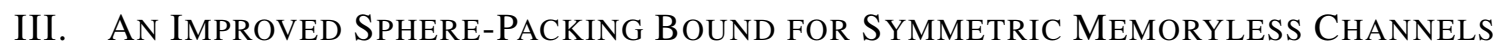

In this section, we derive an improved lower bound on the decoding error probability which utilizes the sphere-packing bounding technique. This new bound is valid for symmetric memoryless channels with a finite input alphabet, and is referred to as an improved sphere-packing (ISP) bound. Note that the symmetry of the channel is crucial for the derivation of the ISP bound in this section, which stays in contrast to the SP67 and VF bounds where channel symmetry is not required. We begin with some necessary definitions and basic properties of symmetric memoryless channels which are used in this section for the derivation of the ISP bound.

\section{A. Symmetric Memoryless Channels}

Definition 3.1: A bijective mapping $g: \mathcal{J} \rightarrow \mathcal{J}$ where $\mathcal{J} \subseteq \mathbb{R}^{d}$ is said to be unitary if for any absolutely integrable generalized function $f: \mathcal{J} \rightarrow \mathbb{R}$

$$
\int_{\mathcal{J}} f(x) \mathrm{d} x=\int_{\mathcal{J}} f(g(x)) \mathrm{d} x
$$

where by generalized function we mean a function which may contain a countable number of shifted Dirac delta functions. If the projection of $\mathcal{J}$ over some of the $d$ dimensions is countable, the integration over these dimensions is turned into a sum.

Remark 3.1: The following properties also hold:

1) If $g$ is a unitary mapping so is its inverse $g^{-1}$.

2) If $\mathcal{J}$ is a countable set, then $g: \mathcal{J} \rightarrow \mathcal{J}$ is unitary if and only if $g$ is bijective.

3) Let $\mathcal{J}$ be an open set and $g: \mathcal{J} \rightarrow \mathcal{J}$ be a bijective function. Denote

$$
g\left(x_{1}, \ldots, x_{d}\right) \triangleq\left(g_{1}\left(x_{1}, \ldots, x_{d}\right), \ldots, g_{d}\left(x_{1}, \ldots, x_{d}\right)\right)
$$

and assume that the partial derivatives $\frac{\partial g_{i}}{\partial x_{j}}$ exist for all $i, j \in\{1,2, \ldots, d\}$. Then $g$ is unitary if and only if the Jacobian satisfies $|J(\mathbf{x})|=1$ for all $\mathbf{x} \in \mathcal{J}$.

Proof: Property 1 follows from (37) and by defining $\widetilde{f}(x) \triangleq f\left(g^{-1}(x)\right)$; this gives

$$
\int_{\mathcal{J}} f\left(g^{-1}(x)\right) \mathrm{d} x=\int_{\mathcal{J}} \widetilde{f}(x) \mathrm{d} x=\int_{\mathcal{J}} \widetilde{f}(g(x)) \mathrm{d} x=\int_{\mathcal{J}} f(x) \mathrm{d} x .
$$

Property 2 follows from the fact that for countable sets, the integral is turned into a sum, and the equality

$$
\sum_{j \in \mathcal{J}} f(j)=\sum_{j \in \mathcal{J}} f(g(j))
$$

holds by a change of order of summation if and only if $g: \mathcal{J} \rightarrow \mathcal{J}$ is bijective (recall that by assumption $\left.\sum_{j \in \mathcal{J}}|f(j)|<\infty\right)$.

Property 3 is proved by a transformation of the integrator in the left side of (37) from $\mathbf{x}=$ $\left(x_{1}, \ldots, x_{d}\right)$ to $g(\mathbf{x})$.

We are now ready to define K-ary input symmetric channels. The symmetry properties of these channels are later exploited to improve the tightness of the sphere-packing bounding technique and derive the ISP lower bound on the average decoding error probability of block codes transmitted over these channels.

Definition 3.2 (Symmetric Memoryless Channels): A memoryless channel with input alphabet $\mathcal{K}=\{0,1, \ldots, K-1\}$, output alphabet $\mathcal{J} \subseteq \mathbb{R}^{d}$ (where $K, d \in \mathbb{N}$ ) and transition probability 
(or density if $\mathcal{J}$ non-countable) function $P(\cdot \mid \cdot)$ is said to be symmetric if there exists a set of unitary mappings $\left\{g_{k}\right\}_{k=0}^{K-1}$ where $g_{k}: \mathcal{J} \rightarrow \mathcal{J}$ for all $k \in \mathcal{K}$ such that

$$
\forall \mathbf{y} \in \mathcal{J}, k \in \mathcal{K}, \quad P(\mathbf{y} \mid 0)=P\left(g_{k}(\mathbf{y}) \mid k\right)
$$

and

$$
\forall k_{1}, k_{2} \in \mathcal{K}, \quad g_{k_{1}}^{-1} \circ g_{k_{2}}=g_{\left(k_{2}-k_{1}\right) \bmod K}
$$

Remark 3.2: From (41), the mapping $g_{0}$ is the identity mapping. Assigning $k_{1}=k$ and $k_{2}=0$ in (42) gives

$$
\forall k \in \mathcal{K} \quad g_{k}^{-1}=g_{(-k) \bmod K}=g_{K-k} .
$$

The class of symmetric memoryless channels, as given in Definition 3.2, is quite large. In particular, it contains the class of memoryless binary-input output-symmetric (MBIOS) channels. To show this, we employ the following proposition which follows from the discussion in [21, Section 4.1.4]:

Proposition 3.1: An MBIOS channel can be equivalently represented as a (time-varying) binary symmetric channel (BSC) whose crossover probabilities for each output symbol are i.i.d. random variables which are independent of the channel inputs, and observed by the receiver. This crossover probability is given by $p=\frac{1}{1+\exp (|L|)}$ where $L=L(y)$ denotes the log-likelihood ratio which corresponds to the channel output $y$.

Corollary 3.1: An arbitrary MBIOS channel can be equivalently represented as a symmetric memoryless channel.

Proof: Let us consider an MBIOS channel $\mathfrak{C}$. Applying Proposition 3.1, it can be equivalently represented by a channel $\mathfrak{C}^{\prime}$ whose output alphabet is $\mathcal{J}=\{0,1\} \times[0,1]$; here, the first term of the output refers to the BSC output and the second term is the associated crossover probability. We now show that this equivalent channel is a symmetric memoryless channel. To this end, it suffices to find a unitary mapping $g_{1}: \mathcal{J} \rightarrow \mathcal{J}$ such that

$$
\forall \mathbf{y} \in \mathcal{J} \quad P(\mathbf{y} \mid 0)=P\left(g_{1}(\mathbf{y}) \mid 1\right)
$$

and $g_{1}^{-1}=g_{1}$.

For the channel $\mathfrak{C}^{\prime \prime}$, the conditional probability distribution (or density) function of the output $\mathbf{y}=(m, p)$ (where $m \in\{0,1\}$ and $p \in[0,1]$ ) given that $i \in\{0,1\}$ is transmitted, is given by

$$
P(\mathbf{y} \mid i)= \begin{cases}\widetilde{P}(p) \cdot(1-p) & \text { if } i=m \\ \widetilde{P}(p) \cdot p & \text { if } i=\bar{m}\end{cases}
$$

where $\widetilde{P}$ is a distribution (or density) over $[0,1]$, and $\bar{m}$ designates the logical not of $m$. From (45), we get that the mapping $g_{1}(m, p)=(\bar{m}, p)$ satisfies (44). Additionally, $g_{1}^{-1}=g_{1}$ since $\overline{\bar{m}}=m$. Therefore, the proof is completed by showing that $g_{1}$ is a unitary mapping. For any (generalized) integrable function $f: \mathcal{J} \rightarrow \mathbb{R}$, we have

$$
\begin{aligned}
\int_{\mathcal{J}} f(\mathbf{x}) \mathrm{d} \mathbf{x} & =\sum_{m=0}^{1} \int_{0}^{1} f(m, p) \mathrm{d} p \\
& =\sum_{m=0}^{1} \int_{0}^{1} f(\bar{m}, p) \mathrm{d} p \\
& =\int_{\mathcal{J}} f\left(g_{1}(\mathbf{x})\right) \mathrm{d} \mathbf{x}
\end{aligned}
$$


where the second equality holds by changing the order of summation; hence, $g_{1}$ is a unitary function.

Remark 3.3: Proposition 3.1 forms a special case of a proposition given in [37, Appendix I]. Using the proposition in [37, Appendix I], which refers to M-ary input channels, it can be shown in a similar way that all M-ary input symmetric-output channels, as defined in [37], can be equivalently represented as symmetric memoryless channels.

Coherently detected M-ary PSK modulated signals transmitted over a fully interleaved fading channel, followed by an additive white Gaussian noise, form another example of a symmetric memoryless channel. In this case, $\mathcal{J}=\mathbb{R}^{2}$ and the mapping $g_{k}$ for $k=0, \ldots, M-1$ forms a clockwise rotation by $\frac{2 \pi k}{M}$ (i.e., $g_{k}(\mathbf{y})=\exp \left(\frac{2 j \pi k}{M}\right) \mathbf{y}$ ). Note that the determinant of the Jacobian of these rotation mappings is equal in absolute value to 1 .

\section{B. Derivation of an Improved Sphere-Packing Bound for Symmetric Memoryless Channels}

In this section, we derive an improved sphere-packing lower bound on the decoding error probability of block codes whose transmission takes place over symmetric memoryless channels. To keep the notation simple, we derive the bound under the assumption that the channel communication is a symmetric DMC. The derivation of the bound is later justified for the extended family of symmetric memoryless channels with discrete or continuous output alphabets.

Though there is a certain parallelism to the derivation of the SP67 bound in [26, Part 1], our analysis for symmetric memoryless channels deviates considerably from the derivation of this classical bound. The improvements suggested in [36] are also incorporated into the derivation of the improved new bound. We show that for symmetric memoryless channels, the derivation of the sphere-packing bound can be modified by skipping the intermediate step of lower bounding the maximal error probability for fixed composition codes, and directly considering the average error probability for general block codes. To this end, the first step of the derivation in [26] (see Theorem 2.1 here) is modified such that instead of bounding the error probability when a single pair of probability assignments is considered, we analyze the average error probability for $M$ pairs of probability assignments.

1) Average Decoding Error Probability for $M$ Pairs of Probability Assignments: We start the analysis by considering the average decoding error probability for $M$ pairs of probability assignments, denoted by $\left\{P_{1}^{m}, P_{2}^{m}\right\}_{m=1}^{M}$, where it is assumed that the index $m$ of the pair is chosen uniformly at random from the set $\{1, \ldots, M\}$ and is known to the decoder (the assumption that the decoder knows the index $m$ is later addressed in Remark 3.5). Denote the observation by $\mathbf{y}$, and the observation space by $\mathcal{Y}$. For simplicity, we assume that $\mathcal{Y}$ is a finite set. Following the notation in [26], the LLR for the $m^{\text {th }}$ pair of probability assignments is

$$
D^{m}(\mathbf{y}) \triangleq \ln \left(\frac{P_{2}^{m}(\mathbf{y})}{P_{1}^{m}(\mathbf{y})}\right)
$$

for all $\mathbf{y} \in \mathcal{Y}$, and define the following probability distribution on $\mathcal{Y}$ :

$$
Q_{s}^{m}(\mathbf{y}) \triangleq \frac{P_{1}^{m}(\mathbf{y})^{1-s} P_{2}^{m}(\mathbf{y})^{s}}{\sum_{\mathbf{y}^{\prime}} P_{1}^{m}\left(\mathbf{y}^{\prime}\right)^{1-s} P_{2}^{m}\left(\mathbf{y}^{\prime}\right)^{s}}, \quad 0<s<1
$$

for $m \in\{1, \ldots, M\}$. For the $m^{\text {th }}$ pair, we also define the function $\mu^{m}$ as

$$
\mu^{m}(s) \triangleq \ln \left(\sum_{\mathbf{y}} P_{1}^{m}(\mathbf{y})^{1-s} P_{2}^{m}(\mathbf{y})^{s}\right), \quad 0<s<1 .
$$


Consider the case where $\mu^{m}$ and its first and second derivatives with respect to $s$ are independent of the value of $m$, and let $\mu \triangleq \mu^{1}=\mu^{2}=\ldots=\mu^{M}$.

Remark 3.4: In the continuation, we will let $P_{2}^{m}$ be a function of $s$. However, the derivatives of $\mu_{m}$ with respect to $s$ will be interpreted as the calculation of the partial derivatives of $\mu_{m}$ with respect to $s$ (hence, $P_{2}^{m}(\mathbf{y})$ is held fixed in (49) when the partial derivative with respect to $s$ is calculated), followed by the substitution of $P_{2}^{m}(\mathbf{y})$ as a function of $s$. We will show that for a specific selection of $P_{1}^{m}$ and $P_{2}^{m}$ which are used to derive the new lower bound on the average block error probability, if the communication takes place over a symmetric memoryless channel, then $\mu^{m}$ and its first two derivatives with respect to $s$ are independent of $m$. Note also that an independence of $\mu^{m}$ in $m$ does not imply that $P_{k}^{m}$ is independent of $m$.

Based on our assumption, in view of (10)-(13), for all $m \in\{1, \ldots, M\}, \mathbf{y} \in \mathcal{Y}$ and $s \in(0,1)$

$$
\begin{aligned}
& \mu^{\prime}(s)=\left(\mu^{m}\right)^{\prime}(s)=\mathbb{E}_{Q_{s}^{m}}\left(D^{m}(\mathbf{y})\right) \\
& \mu^{\prime \prime}(s)=\left(\mu^{m}\right)^{\prime \prime}(s)=\operatorname{Var}_{Q_{s}^{m}}\left(D^{m}(\mathbf{y})\right) \\
& P_{1}^{m}(\mathbf{y})=\exp \left(\mu(s)-s D^{m}(\mathbf{y})\right) Q_{s}^{m}(\mathbf{y}) \\
& P_{2}^{m}(\mathbf{y})=\exp \left(\mu(s)+(1-s) D^{m}(\mathbf{y})\right) Q_{s}^{m}(\mathbf{y})
\end{aligned}
$$

where $\mathbb{E}_{Q}$ and $\operatorname{Var}_{Q}$ stand, respectively, for the statistical expectation and variance with respect to a probability distribution $Q$. For the $m^{\text {th }}$ pair of probability assignments $\left(P_{1}^{m}, P_{2}^{m}\right)$, the set of typical output vectors is defined as

$$
\mathcal{Y}_{s}^{m, x} \triangleq\left\{\mathbf{y} \in \mathcal{Y}:\left|D^{m}(\mathbf{y})-\mu^{\prime}(s)\right| \leq x \sqrt{2 \mu^{\prime \prime}(s)}\right\}
$$

where $x>0$ is a free parameter. In the original derivation of the SP67 bound in [26] (see (14) here), the parameter $x$ is set to 1 ; similarly to [36], this free parameter is introduced in (54) in order to tighten the bound for finite-length block codes. However, in both [26] and [36], only one pair of probability assignments was considered. By applying Chebychev's inequality to (54), we get from (50) and (51) that for all $m \in\{1, \ldots, M\}$

$$
\sum_{\mathbf{y} \in \mathcal{Y}_{s}^{m, x}} Q_{s}^{m}(\mathbf{y})>1-\frac{1}{2 x^{2}}
$$

where (55) is meaningful only for $x>\frac{\sqrt{2}}{2}$.

Since the index $m$ is known to the decoder, $P_{1}^{m}$ is decoded only against $P_{2}^{m}$. Let $\mathcal{Y}_{1}^{m}$ and $\mathcal{Y}_{2}^{m}$ be, respectively, the decoding regions of $P_{1}^{m}$ and $P_{2}^{m}$; hence, $\mathcal{Y}_{1}^{m}$ and $\mathcal{Y}_{2}^{m}$ form a partition of the observation space $\mathcal{Y}$. We now derive a lower bound on the conditional error probability, given that the correct hypothesis is the first probability assignment and the $m^{\text {th }}$ pair was selected. Similarly to (15) and (16), we get the following lower bounds from (52)-(54):

$$
\begin{aligned}
& P_{\mathrm{e}, 1}^{m} \geq \exp \left(\mu(s)-s \mu^{\prime}(s)-s x \sqrt{2 \mu^{\prime \prime}(s)}\right) \sum_{\mathbf{y} \in \mathcal{Y}_{2}^{m} \cap \mathcal{Y}_{s}^{m, x}} Q_{s}^{m}(\mathbf{y}), \\
& P_{\mathrm{e}, 2}^{m} \geq \exp \left(\mu(s)+(1-s) \mu^{\prime}(s)-(1-s) x \sqrt{2 \mu^{\prime \prime}(s)}\right) \sum_{\mathbf{y} \in \mathcal{Y}_{1}^{m} \cap \mathcal{Y}_{s}^{m, x}} Q_{s}^{m}(\mathbf{y}) .
\end{aligned}
$$


Averaging (56) and (57) over $m$ gives that for all $s \in(0,1)$

$$
\begin{aligned}
P_{\mathrm{e}, 1}^{\mathrm{avg}} & \triangleq \frac{1}{M} \sum_{m=1}^{M} P_{\mathrm{e}, 1}^{m} \\
& \geq \exp \left(\mu(s)-s \mu^{\prime}(s)-s x \sqrt{2 \mu^{\prime \prime}(s)}\right) \frac{1}{M} \sum_{m=1}^{M} \sum_{\mathbf{y} \in \mathcal{Y}_{2}^{m} \cap \mathcal{Y}_{s}^{m, x}} Q_{s}^{m}(\mathbf{y}) \\
P_{\mathrm{e}, 2}^{\mathrm{avg}} & \triangleq \frac{1}{M} \sum_{m=1}^{M} P_{\mathrm{e}, 2}^{m} \\
& \geq \exp \left(\mu(s)+(1-s) \mu^{\prime}(s)-(1-s) x \sqrt{2 \mu^{\prime \prime}(s)}\right) \frac{1}{M} \sum_{m=1}^{M} \sum_{\mathbf{y} \in \mathcal{Y}_{1}^{m} \cap \mathcal{Y}_{s}^{m, x}} Q_{s}^{m}(\mathbf{y})
\end{aligned}
$$

where $P_{\mathrm{e}, 1}^{\mathrm{avg}}$ and $P_{\mathrm{e}, 2}^{\mathrm{avg}}$ refer to the average error probabilities given that the first or second hypotheses, respectively, of a given pair are correct where this pair is chosen uniformly at random among the $M$ possible pairs of hypotheses. Since for all $m$, the sets $\mathcal{Y}_{1}^{m}$ and $\mathcal{Y}_{2}^{m}$ form a partition of the set of output vectors $\mathcal{Y}$, then

$$
\begin{aligned}
& \frac{1}{M} \sum_{m=1}^{M} \sum_{\mathbf{y} \in \mathcal{Y}_{1}^{m} \cap \mathcal{Y}_{s}^{m, x}} Q_{s}^{m}(\mathbf{y})+\frac{1}{M} \sum_{m=1}^{M} \sum_{\mathbf{y} \in \mathcal{Y}_{2}^{m} \cap \mathcal{Y}_{s}^{m, x}} Q_{s}^{m}(\mathbf{y}) \\
& =\frac{1}{M} \sum_{m=1}^{M} \sum_{\mathbf{y} \in \mathcal{Y}_{s}^{m, x}} Q_{s}^{m}(\mathbf{y}) \\
& >1-\frac{1}{2 x^{2}}
\end{aligned}
$$

where the last transition follows from (55) and is meaningful for $x>\frac{\sqrt{2}}{2}$. Hence, at least one of the terms in the left side of the above equality is necessarily greater than $\frac{1}{2}\left(1-\frac{1}{2 x^{2}}\right)$. Combining this result with (58) and (59), we get that for every $s \in(0,1)$

$$
P_{\mathrm{e}, 1}^{\mathrm{avg}}>\left(\frac{1}{2}-\frac{1}{4 x^{2}}\right) \exp \left(\mu(s)-s \mu^{\prime}(s)-s x \sqrt{2 \mu^{\prime \prime}(s)}\right)
$$

or

$$
P_{\mathrm{e}, 2}^{\mathrm{avg}}>\left(\frac{1}{2}-\frac{1}{4 x^{2}}\right) \exp \left(\mu(s)+(1-s) \mu^{\prime}(s)-(1-s) x \sqrt{2 \mu^{\prime \prime}(s)}\right) .
$$

Eqs. (61) and (62) provide lower bounds on the average conditional error probabilities for $M$ pairs of probability assignments.

Remark 3.5: The assumption whereby the index $m$ of the pair of probability assignments is known to the decoder is only used in the above derivation to justify that $\mathcal{Y}_{1}^{m}=\left(\mathcal{Y}_{2}^{m}\right)^{\mathrm{c}}$ for all $m$ (since by letting the decoder know the index $m$, the probability assignment $P_{1}^{m}$ is decoded only against $P_{2}^{m}$, and the decision regions $\mathcal{Y}_{1}^{m}$ and $\mathcal{Y}_{2}^{m}$ form a partition of the observation space). Eqs. (61) and (62) are valid in general as lower bounds on the average conditional error probabilities for any set of $M$ pairs of probability assignments, as long as the function $\mu^{m}$ in (49) and its first and second derivatives are independent of the index $m$. In the continuation, we apply (61) and (62) to derive a lower bound on the average decoding error probability of general block codes which are decoded by an arbitrary list decoder. To this end, $m$ is used to index the codewords, $P_{1}^{m}$ is set to the distribution of the channel output when codeword number $m$ is transmitted, and $P_{2}^{m}$ is set to some specific probability distribution calculated from the channel 
statistics. The regions $\mathcal{Y}_{1}^{m}$ and $\mathcal{Y}_{2}^{m}$ are set so that the condition $\mathcal{Y}_{1}^{m}=\left(\mathcal{Y}_{2}^{m}\right)^{\mathrm{c}}$ holds for all $m$. In this setting, the transmitted codeword is not known to the decoder. However, the assignment of the regions $\mathcal{Y}_{1}^{m}$ and $\mathcal{Y}_{2}^{m}$, which are set to be disjoint and to partition the observation space, ensures that the inequalities (61) and (62) can be applied in this setting (after verifying that the requirement on the independence of $\mu^{m}$ and its first two derivatives on the index $m$ holds).

We now turn to consider a block code which is transmitted over a symmetric DMC. Similarly to the derivation of the SP67 bound in [26], we use the lower bound derived in this section to relate the decoding error probability when a given codeword is transmitted to the size of the decision region associated with this codeword. However, the bound above allows us to directly consider the average block error probability; this is in contrast to the derivation in [26] which first considered the maximal block error probability of the code and then used an argument based on expurgating half of the bad codewords in order to obtain a lower bound on the average error probability of the original code (where the code rate is asymptotically not affected as a result of this expurgation). Additionally, we show that when the transmission takes place over a memoryless symmetric channel, one can consider directly an arbitrary block code instead of starting the analysis by referring to fixed composition codes as in [26, Part 1] and [36].

2) Lower Bound on the Decoding Error Probability of General Block Codes: We now consider a block code $\mathcal{C}$ of length $N$ with $M$ codewords, denoted by $\left\{\mathbf{x}_{m}\right\}_{m=1}^{M}$; assume that the transmission takes place over a symmetric DMC with transition probabilities $P(j \mid k)$, where $k \in \mathcal{K}=\{0, \ldots, K-1\}$ and $j \in \mathcal{J}=\{0, \ldots, J-1\}$ designate the channel input and output alphabets, respectively. In this section, we derive a lower bound on the average block error probability of the code $\mathcal{C}$ for an arbitrary list decoder where the size of the list is limited to $L$. Let $f_{N}$ be a probability measure defined over the set of length- $N$ sequences of the channel output, and which can be factorized as in (19). We define $M$ pairs of probability measures $\left\{P_{1}^{m}, P_{2}^{m}\right\}$ by

$$
P_{1}^{m}(\mathbf{y}) \triangleq \operatorname{Pr}\left(\mathbf{y} \mid \mathbf{x}_{m}\right), \quad P_{2}^{m}(\mathbf{y}) \triangleq f_{N}(\mathbf{y}), \quad m \in\{1,2, \ldots, M\}
$$

where $\mathbf{x}_{m}$ is the $m^{\text {th }}$ codeword of the code $\mathcal{C}$. Combining (49) and (63), the function $\mu^{m}$ takes the form

$$
\mu^{m}(s)=\ln \left(\sum_{\mathbf{y}} \operatorname{Pr}\left(\mathbf{y} \mid \mathbf{x}_{m}\right)^{1-s} f_{N}(\mathbf{y})^{s}\right), \quad 0<s<1 .
$$

Let us denote by $q_{k}^{m}$ the fraction of appearances of the letter $k$ in the codeword $\mathbf{x}_{m}$. By assumption, the communication channel is memoryless and the function $f_{N}$ is a probability measure which is factorized according to (19). Hence, for every $m \in\{1,2, \ldots, M\}$, the function $\mu^{m}$ in (64) is expressible in the form

$$
\mu^{m}(s)=N \sum_{k=0}^{K-1} q_{k}^{m} \mu_{k}(s)
$$

where

$$
\mu_{k}(s) \triangleq \ln \left(\sum_{j=0}^{J-1} P(j \mid k)^{1-s} f(j)^{s}\right), \quad 0<s<1 .
$$

In order to validate the statement which assures that at least one of the inequalities in (61) and (62) is satisfied, it is required to verify in this case that the function $\mu^{m}$ and its first and second derivatives with respect to $s$ are independent of the index $m$. From (65), since $\sum_{k=0}^{K-1} q_{k}^{m}=1$ for every $m \in\{1, \ldots, M\}$, it suffices to show that $\mu_{k}$ and its first and second derivatives are 
independent of the input symbol $k$. To this end, for every $s \in(0,1)$, we choose the function $f$ to be $f_{s}$, as given in [26, Eqs. (4.18)-(4.20)]. Namely, for $0<s<1$, let $\mathbf{q}_{s}=\left\{q_{0, s}, \ldots, q_{K-1, s}\right\}$ satisfy the inequalities

$$
\sum_{j=0}^{J-1} P(j \mid k)^{1-s} \alpha_{j, s}^{\frac{s}{1-s}} \geq \sum_{j=0}^{J-1} \alpha_{j, s}^{\frac{1}{1-s}}, \quad \forall k \in \mathcal{K}
$$

where

$$
\alpha_{j, s} \triangleq \sum_{k^{\prime}=0}^{K-1} q_{k^{\prime}, s} P\left(j \mid k^{\prime}\right)^{1-s} .
$$

The function $f=f_{s}$ is given by

$$
f_{s}(j)=\frac{\alpha_{j, s}^{\frac{1}{1-s}}}{\sum_{j^{\prime}=0}^{J-1} \alpha_{j^{\prime}, s}^{\frac{1}{1-s}}}, \quad j \in\{0, \ldots, J-1\} .
$$

Note that the input distribution $\mathbf{q}_{s}$ is independent of the code $\mathcal{C}$, as it only depends on the channel statistics. It should be also noted that $P_{1}^{m}$ and $P_{2}^{m}$ are in general allowed to depend on the parameter $s$, though the differentiation of the function $\mu^{m}$ with respect to $s$ is performed while holding $P_{1}^{m}$ and $P_{2}^{m}$ fixed. The following lemma shows that for symmetric channels, the function $f_{s}$ in (69) yields that $\mu_{k}$ and its first and second derivatives with respect to $s$ (while holding $f_{s}$ fixed) are independent of the input symbol $k$.

Lemma 3.1: Let $P(\cdot \mid \cdot)$ designate the transition probability function of a symmetric DMC with input alphabet $\mathcal{K}=\{0, \ldots, K-1\}$ and output alphabet $\mathcal{J}=\{0, \ldots, J-1\}$, and let $\mu_{k}$ be defined as in (66), where $f=f_{s}$ is given in (69). Then, the following properties hold for all $s \in(0,1)$ :

$$
\begin{aligned}
& \mu_{0}(s)=\mu_{1}(s)=\ldots=\mu_{K-1}(s)=-(1-s) E_{0}\left(\frac{s}{1-s}\right) \\
& \mu_{0}^{\prime}(s)=\mu_{1}^{\prime}(s)=\ldots=\mu_{K-1}^{\prime}(s) \\
& \mu_{0}^{\prime \prime}(s)=\mu_{1}^{\prime \prime}(s)=\ldots=\mu_{K-1}^{\prime \prime}(s)
\end{aligned}
$$

where $E_{0}$ is introduced in (25), and the differentiation in (71) and (72) is performed in (66) with respect to $s$ while holding $f=f_{s}$ in (69) fixed.

Proof: See Appendix A.

Remark 3.6: Since the differentiation of the function $\mu_{k}$ with respect to $s$ is performed while holding $f=f_{s}$ fixed, the independence of $\mu_{k}$ in $k$ (see (70)) does not necessarily imply the independence in $k$ of its first and second derivatives (see (71) and (72)). In order to prove Lemma 3.1 (see Appendix A), we rely on the symmetry of the memoryless channel. The function $\mu_{0}$ in (5) and its derivatives are calculated in Appendix B for some symmetric memoryless channels, and these are used in Section V for numerical calculations of sphere-packing bounds.

In view of (65) and Lemma 3.1, it follows that the function $\mu^{m}$ and its first and second derivatives with respect to $s$ are independent of the index $m$ (where this property also follows since $\sum_{k=0}^{K-1} q_{k}^{m}=1$ irrespectively of $m$ ).

Let $\mathcal{Y}_{m}$ be the decision region of the codeword $\mathbf{x}_{m}$. By associating $\mathcal{Y}_{m}$ and $\mathcal{Y}_{m}^{\mathrm{c}}$ with the two decision regions for the probability measures $P_{1}^{m}$ and $P_{2}^{m}$, respectively, we get from (63)

$$
P_{\mathrm{e}, 1}^{m}=\sum_{\mathbf{y} \in \mathcal{Y}_{m}^{c}} P_{1}^{m}(\mathbf{y})=\sum_{\mathbf{y} \in \mathcal{Y}_{m}^{c}} \operatorname{Pr}\left(\mathbf{y} \mid \mathbf{x}_{m}\right) \triangleq P_{\mathrm{e}, m}
$$


and

$$
P_{\mathrm{e}, 2}^{m}=\sum_{\mathbf{y} \in \mathcal{Y}_{m}} P_{2}^{m}(\mathbf{y})=\sum_{\mathbf{y} \in \mathcal{Y}_{m}} f_{N}(\mathbf{y})=F\left(\mathcal{Y}_{m}\right)
$$

where $P_{\mathrm{e}, m}$ is the decoding error probability of the code $\mathcal{C}$ when the codeword $\mathbf{x}_{m}$ is transmitted, and $F\left(\mathcal{Y}_{m}\right)$ is a measure for the size of the decoding region $\mathcal{Y}_{m}$ as defined in (20). Combining (61), (62), (73) and (74) yields that for every $s \in(0,1)$

$$
\frac{1}{M} \sum_{m=1}^{M} P_{\mathrm{e}, m}=P_{\mathrm{e}, 1}^{\mathrm{avg}}>\left(\frac{1}{2}-\frac{1}{4 x^{2}}\right) \exp \left(\mu(s)-s \mu^{\prime}(s)-s x \sqrt{2 \mu^{\prime \prime}(s)}\right)
$$

or

$$
\frac{1}{M} \sum_{m=1}^{M} F_{s}\left(\mathcal{Y}_{m}\right)=P_{\mathrm{e}, 2}^{\mathrm{avg}}>\left(\frac{1}{2}-\frac{1}{4 x^{2}}\right) \exp \left(\mu(s)+(1-s) \mu^{\prime}(s)-(1-s) x \sqrt{2 \mu^{\prime \prime}(s)}\right)
$$

where $x>\frac{\sqrt{2}}{2}$ and $F_{s}\left(\mathcal{Y}_{m}\right) \triangleq \sum_{\mathbf{y} \in \mathcal{Y}_{m}} f_{N, s}(\mathbf{y})$. Similarly to [26], we relate $\sum_{m=1}^{M} F_{s}\left(\mathcal{Y}_{m}\right)$ to the number of codewords $M$ and the size of the decoding list which is limited to $L$. First, for all $0 \leq s \leq 1$

$$
\sum_{m=1}^{M} F_{s}\left(\mathcal{Y}_{m}\right)=\sum_{m=1}^{M} \sum_{\mathbf{y} \in \mathcal{Y}_{m}} f_{N, s}(\mathbf{y}) \leq L
$$

where the last inequality holds since each $\mathbf{y} \in \mathcal{J}^{N}$ is included in at most $L$ subsets $\left\{\mathcal{Y}_{m}\right\}_{m=1}^{M}$ and $\sum_{\mathbf{y}} f_{N, s}(\mathbf{y})=1$. Hence, the left side of (76) is upper bounded by $\frac{L}{M}$ for all $0 \leq s \leq 1$. Additionally, the left side of (75) is equal by definition to the average block error probability $P_{\mathrm{e}}$ of the code $\mathcal{C}$. Therefore, (75) and (76) can be rewritten as

$$
P_{\mathrm{e}}>\left(\frac{1}{2}-\frac{1}{4 x^{2}}\right) \exp \left(\mu(s)-s \mu^{\prime}(s)-s x \sqrt{2 \mu^{\prime \prime}(s)}\right)
$$

or

$$
\frac{L}{M}>\left(\frac{1}{2}-\frac{1}{4 x^{2}}\right) \exp \left(\mu(s)+(1-s) \mu^{\prime}(s)-(1-s) x \sqrt{2 \mu^{\prime \prime}(s)}\right) .
$$

Applying (65) and Lemma 3.1 to (78) and (79) gives that for all $s \in(0,1)$

$$
P_{\mathrm{e}}>\left(\frac{1}{2}-\frac{1}{4 x^{2}}\right) \exp \left\{N\left(\mu_{0}\left(s, f_{s}\right)-s \mu_{0}^{\prime}\left(s, f_{s}\right)-s x \sqrt{\frac{2 \mu_{0}^{\prime \prime}\left(s, f_{s}\right)}{N}}\right)\right\}
$$

or

$$
\frac{L}{M}>\left(\frac{1}{2}-\frac{1}{4 x^{2}}\right) \exp \left\{N\left(\mu_{0}\left(s, f_{s}\right)+(1-s) \mu_{0}^{\prime}\left(s, f_{s}\right)-(1-s) x \sqrt{\frac{2 \mu_{0}^{\prime \prime}\left(s, f_{s}\right)}{N}}\right)\right\} .
$$

A lower bound on the average block error probability can be obtained from (80) by substituting any value of $s \in(0,1)$ for which (81) does not hold. In particular we choose a value $s=s_{x}$ 
such that the inequality in (81) is replaced by an equality, i.e.,

$$
\begin{array}{r}
\frac{L}{M}=\exp (-N R) \\
=\left(\frac{1}{2}-\frac{1}{4 x^{2}}\right) \exp \left\{N \left(\mu_{0}\left(s_{x}, f_{s_{x}}\right)+\left(1-s_{x}\right) \mu_{0}^{\prime}\left(s_{x}, f_{s_{x}}\right)\right.\right. \\
\left.\left.\quad-\left(1-s_{x}\right) x \sqrt{\frac{2 \mu_{0}^{\prime \prime}\left(s_{x}, f_{s_{x}}\right)}{N}}\right)\right\}
\end{array}
$$

where $R \triangleq \frac{\ln \left(\frac{M}{L}\right)}{N}$ designates the code rate in nats per channel use. Note that the existence of a solution $s=s_{x}$ to (82) can be demonstrated in a similar way to the arguments in [26, Eqs. (4.28)-(4.35)] for the non-trivial case where the sphere-packing bound does not reduce to the trivial inequality $P_{\mathrm{e}} \geq 0$. This particular value of $s$ is chosen since for a large enough value of $N$, the right side of (80) is monotonically decreasing while the right side of (81) is monotonically increasing for $s \in(0,1)$; thus, this choice is optimal for large enough $N$. The choice of $s=s_{x}$ also allows to get a simpler representation of the bound on the average block error probability. Rearranging (82) gives

$$
\mu_{0}^{\prime}\left(s_{x}, f_{s_{x}}\right)=-\frac{1}{1-s_{x}}\left[R+\mu_{0}\left(s_{x}, f_{s_{x}}\right)+\frac{1}{N} \ln \left(\frac{1}{2}-\frac{1}{4 x^{2}}\right)\right]+x \sqrt{\frac{2 \mu_{0}^{\prime \prime}\left(s_{x}, f_{s_{x}}\right)}{N}} .
$$

Substituting $s=s_{x}$ and (83) into (80) yields

$$
\begin{array}{r}
P_{\mathrm{e}}>\exp \left\{N \left(\frac{\mu_{0}\left(s_{x}, f_{s_{x}}\right)}{1-s_{x}}+\frac{s_{x}}{1-s_{x}}\left[R+\frac{1}{N} \ln \left(\frac{1}{2}-\frac{1}{4 x^{2}}\right)\right]\right.\right. \\
\left.\left.-s_{x} x \sqrt{\frac{8 \mu_{0}^{\prime \prime}\left(s_{x}, f_{s_{x}}\right)}{N}}+\frac{1}{N} \ln \left(\frac{1}{2}-\frac{1}{4 x^{2}}\right)\right)\right\} .
\end{array}
$$

In view of (70), setting $\rho_{x} \triangleq \frac{s_{x}}{1-s_{x}}$ yields

$$
\begin{aligned}
P_{\mathrm{e}}>\exp \{-N & \left(E_{0}\left(\rho_{x}\right)-\rho_{x}\left[R+\frac{1}{N} \ln \left(\frac{1}{2}-\frac{1}{4 x^{2}}\right)\right]\right. \\
& \left.\left.+s_{x} x \sqrt{\frac{8 \mu_{0}^{\prime \prime}\left(s_{x}, f_{s_{x}}\right)}{N}}-\frac{1}{N} \ln \left(\frac{1}{2}-\frac{1}{4 x^{2}}\right)\right)\right\} .
\end{aligned}
$$

Note that (85) holds for every block code of length $N$ and rate $R$. The choice of $\rho_{x}$ is similar to [36], and the parameter $x \in\left(\frac{\sqrt{2}}{2}, \infty\right)$ is optimized to obtain the tightest bound in (85).

The above derivation only relies on the fact that the channel is memoryless and symmetric, but the output alphabet does not need to be discrete. As mentioned in Section II-B, the original derivation of the SP67 bound in [26] assumes that the input and output alphabets are finite in order to upper bound $\mu^{\prime \prime}(s)$ by $\left(\frac{1}{s} \ln \left(\frac{e}{\sqrt{P_{\min }}}\right)\right)^{2}$ where $P_{\min }$ designates the smallest non-zero transition probability of the channel. This requirement was relaxed in [36] to the requirement that only the input alphabet is finite; to this end, the second derivative of the function $\mu$ is calculated, thus the above upper bound on this second derivative is replaced by its exact value. The validity of the derivation for memoryless symmetric channels with continuous-output alphabets is addressed in the continuation (see Remark 3.9). This leads to the following theorem, providing an improved sphere-packing lower bound on the decoding error probability of block codes which are transmitted over symmetric memoryless channels. 
Theorem 3.1 (An Improved Sphere-Packing (ISP) Bound for Symmetric Memoryless Channels): Let $\mathcal{C}$ be an arbitrary block code consisting of $M$ codewords, each of length $N$. Assume that $\mathcal{C}$ is transmitted over a memoryless symmetric channel which is specified by the transition probabilities (or densities) $P(j \mid k)$ where $k \in \mathcal{K}=\{0, \ldots, K-1\}$ and $j \in \mathcal{J} \subseteq \mathbb{R}^{d}$ designate the channel input and output alphabets, respectively. Assume an arbitrary list decoder where the size of the list is limited to $L$. Then, the average decoding error probability satisfies

$$
P_{\mathrm{e}}(N, M, L) \geq \exp \left(-N E_{\mathrm{ISP}}(R, N)\right)
$$

where

$$
E_{\mathrm{ISP}}(R, N) \triangleq \inf _{x>\frac{\sqrt{2}}{2}}\left\{E_{0}\left(\rho_{x}\right)-\rho_{x}\left(R-O_{1}\left(\frac{1}{N}, x\right)\right)+O_{2}\left(\frac{1}{\sqrt{N}}, x, \rho_{x}\right)\right\}
$$

the function $E_{0}$ is introduced in (25), $R=\frac{1}{N} \ln \left(\frac{M}{L}\right)$, and

$$
\begin{aligned}
& O_{1}\left(\frac{1}{N}, x\right) \triangleq-\frac{1}{N} \ln \left(\frac{1}{2}-\frac{1}{4 x^{2}}\right) \\
& O_{2}\left(\frac{1}{\sqrt{N}}, x, \rho\right) \triangleq s(\rho) x \sqrt{\frac{8}{N} \mu_{0}^{\prime \prime}\left(s(\rho), f_{s(\rho)}\right)}-\frac{1}{N} \ln \left(\frac{1}{2}-\frac{1}{4 x^{2}}\right) .
\end{aligned}
$$

Here, $s(\rho) \triangleq \frac{\rho}{1+\rho}$, and the non-negative parameter $\rho=\rho_{x}$ in the right side of (87) is determined by solving the equation

$$
\begin{aligned}
R-O_{1}\left(\frac{1}{N}, x\right)= & -\mu_{0}\left(s(\rho), f_{s(\rho)}\right)-(1-s(\rho)) \mu_{0}^{\prime}\left(s(\rho), f_{s(\rho)}\right) \\
& +(1-s(\rho)) x \sqrt{\frac{2 \mu_{0}^{\prime \prime}\left(s(\rho), f_{s(\rho)}\right)}{N}}
\end{aligned}
$$

with the functions $\mu_{0}(s, f)$ and $f_{s}$ which are defined in (66) and (69), respectively.

Remark 3.7: The requirement that the memoryless communication channel is symmetric is crucial to the derivation of the ISP bound. One of the new concepts introduced here is the use of the channel symmetry to show that the function $\mu^{m}$ and its first and second derivatives with respect to $s$ are independent of the codeword composition. This enables to tighten the VF bound in [36] by skipping the intermediate step which is related to fixed composition codes. Another new concept is a direct consideration of the average decoding error probability of the code rather than considering the maximal block error probability and expurgating the code. This is due to the consideration of $M$ pairs of probability distributions in the first step of the derivation. Note that the bound on the average block error probability of $M$ pairs of probability assignments requires that $\mu^{m}$ and its first and second derivatives are independent of the index $m$; this property holds due to the symmetry of the memoryless communication channel.

Remark 3.8: In light of Remark 3.7 whereby it is not needed to consider the block error probability of fixed composition codes as an intermediate step when the memoryless channel is symmetric, the ISP bound differs from the VF bound [36] (see Theorem 2.4) in the sense that the term $\frac{\log \left(\begin{array}{c}N+K-1 \\ K-1\end{array}\right)}{N}$ is removed from $O_{1}\left(\frac{\ln N}{N}, x\right)$ (see (31)). Therefore, the shift in the rate of the error exponent of the ISP bound scales asymptotically like $\frac{1}{N}$ instead of $\frac{\ln N}{N}$ (see (28), (31) and (88)). Additionally, the derivation of the VF bound requires expurgation of the code to transform a lower bound on the maximal block error probability to a lower bound on the average block error probability. These differences indicate a tightening of the pre-exponent of the ISP bound (as compared to the SP67 and VF bounds) which is expected to be especially 
pronounced for codes of small to moderate block lengths and also when the size of the channel input alphabet is large (as is later verified in Section V).

Remark 3.9: The ISP bound is also applicable to memoryless symmetric channels with a continuous-output alphabet. When the ISP bound is applied to such a channel, the transition probability is replaced by a transition density function and sums over the output alphabet are replaced by integrals; note that these densities may include Dirac delta functions. In view of Appendix A, the statement in Lemma 3.1 holds for general symmetric memoryless channels.

\section{The 1959 Sphere-Packing Bound of Shannon And Improved Algorithms for ITS CALCULATION}

The 1959 sphere-packing (SP59) bound by Shannon [25] is a lower bound on the decoding error probability of an arbitrary block code which is modulated by equal-energy signals whose transmission takes place over an AWGN channel. We start this section by introducing the SP59 bound, along with its asymptotic approximations in [25] for large block lengths. We then review a theorem from [36] which provides recursive equations for simplifying the calculation of this bound. Both the original SP59 bound in [25] and the recursions in [36] involve calculations which lead to numerical difficulties of over and under flows for block lengths of $N \geq 1000$. In this section, we present an alternative approach which calculates the SP59 bound in the logarithmic domain; this method circumvents the numerical problems in the calculation of the SP59 bound regardless of the block length.

\section{A. The 1959 Sphere-Packing Bound and Asymptotic Approximations}

Consider a block code $\mathcal{C}$ of length $N$ and rate $R$ nats per channel use per dimension. It is assumed that all the codewords are mapped to signals with equal energy (e.g., PSK modulation); hence, all the signals representing codewords lie on an $N$-dimensional sphere centered at the origin, but finer details of the modulation used are not taken into account in the derivation of the bound. This assumption implies that every Voronoi cell (i.e., the convex region containing all the points which are closer to the considered signal than to any other code signal) is a polyhedric cone which is limited by at most $\exp (N R)-1$ hyper planes intersecting at the origin. As a measure of volume, Shannon introduced the solid angle of a cone which is defined to be the area of the sphere of unit radius cut out by the cone. Since the Voronoi cells partition the space $\mathbb{R}^{N}$, then the sum of their solid angles is equal to the area of an $N$-dimensional sphere of unit radius. The derivation of the SP59 bound relies on two main observations:

- Among the cones of a given solid angle, the lowest probability of error is obtained by the circular cone whose main axis passes through the origin and the signal point which represents the transmitted signal.

- In order to minimize the average decoding error probability, it is best to share the total solid angle equally among the $\exp (N R)$ Voronoi regions.

As a corollary of these two observations, it follows that the average block error probability cannot be smaller than the error probability which corresponds to the case where all the Voronoi regions are circular cones centered around the code signals with a common solid angle which is equal to a fraction of $\exp (-N R)$ of the solid angle of $\mathbb{R}^{N}$. The solid angle of a circular cone is given by the following lemma.

Lemma 4.1 (Solid Angle of a Circular Cone [25]): The solid angle of a circular cone of half angle $\theta$ in $\mathbb{R}^{N}$ is given by

$$
\Omega_{N}(\theta)=\frac{2 \pi^{\frac{N-1}{2}}}{\Gamma\left(\frac{N-1}{2}\right)} \int_{0}^{\theta} \sin ^{N-2} \phi \mathrm{d} \phi .
$$


In particular, the solid angle of $\mathbb{R}^{N}$ is given by

$$
\Omega_{N}(\pi)=\frac{2 \pi^{\frac{N}{2}}}{\Gamma\left(\frac{N}{2}\right)} .
$$

Theorem 4.1 (The 1959 Sphere-Packing (SP59) Bound [25]): Assume that the codewords of an arbitrary block code of length $N$ and rate $R$ (in nats per channel use) are modulated by equal-energy signals and transmitted over an AWGN channel. Let $E_{\mathrm{s}}$ be the energy per symbol of these modulated signals, and let $N_{0}$ be the one-sided spectral density of the white Gaussian noise. Then, under ML decoding, the block error probability satisfies

$$
P_{\mathrm{e}}>P_{\mathrm{SPB}}(N, \theta, A)
$$

where

$$
\begin{aligned}
& P_{\mathrm{SPB}}(N, \theta, A) \triangleq \frac{(N-1) \exp \left(-\frac{N A^{2}}{2}\right)}{\sqrt{2 \pi}} \int_{\theta}^{\frac{\pi}{2}} \sin ^{N-2} \phi f_{N}(\sqrt{N} A \cos \phi) \mathrm{d} \phi+Q(\sqrt{N} A), \\
& f_{N}(x) \triangleq \frac{1}{2^{\frac{N-1}{2}} \Gamma\left(\frac{N+1}{2}\right)} \int_{0}^{\infty} z^{N-1} \exp \left(-\frac{z^{2}}{2}+z x\right) \mathrm{d} z, \quad \forall x \in \mathbb{R}, N \in \mathbb{N} \\
& A \triangleq \sqrt{\frac{2 E_{\mathrm{s}}}{N_{0}}}
\end{aligned}
$$

and, with (91) and (92), $\theta \in[0, \pi]$ satisfies

$$
\frac{\Omega_{N}(\theta)}{\Omega_{N}(\pi)} \geq \exp (-N R)
$$

By assumption, the transmitted signal is represented by a point which lies on the surface of an $N$-dimensional sphere which is centered at the origin with radius $\sqrt{N E_{\mathrm{s}}}$, and the Gaussian noise is additive. The value of $P_{\mathrm{SPB}}(N, \theta, A)$ in the right side of (93) designates the probability that the received vector falls outside the $N$-dimensional circular cone of half angle $\theta$ whose main axis passes through the origin and the signal point which represents the transmitted signal. Hence, this function is monotonically decreasing in $\theta$. The tightest lower bound on the decoding error probability, as given in (93), is therefore achieved for $\theta_{1}(N, R)$ which satisfies

$$
\frac{\Omega_{N}\left(\theta_{1}(N, R)\right)}{\Omega_{N}(\pi)}=\exp (-N R) .
$$

In order to simplify the calculation of the SP59 bound, Shannon provided in [25] asymptotically tight upper and lower bounds on the ratio $\frac{\Omega_{N}(\theta)}{\Omega_{N}(\pi)}$.

Lemma 4.2 (Bounds on the Solid Angle [25]): The solid angle of a circular cone of half angle $\theta$ in the Euclidean space $\mathbb{R}^{N}$ satisfies the inequality

$$
\frac{\Gamma\left(\frac{N}{2}\right) \sin ^{N-1} \theta}{2 \Gamma\left(\frac{N+1}{2}\right) \sqrt{\pi} \cos \theta}\left(1-\frac{\tan ^{2} \theta}{N}\right) \leq \frac{\Omega_{N}(\theta)}{\Omega_{N}(\pi)} \leq \frac{\Gamma\left(\frac{N}{2}\right) \sin ^{N-1} \theta}{2 \Gamma\left(\frac{N+1}{2}\right) \sqrt{\pi} \cos \theta} .
$$

Corollary 4.1 (SP59 Bound (Cont.)): If $\theta^{*}$ satisfies the equality

$$
\frac{\Gamma\left(\frac{N}{2}\right) \sin ^{N-1} \theta^{*}}{2 \Gamma\left(\frac{N+1}{2}\right) \sqrt{\pi} \cos \theta^{*}}\left(1-\frac{\tan ^{2} \theta^{*}}{N}\right)=\exp (-N R)
$$


then $\frac{\Omega_{N}\left(\theta^{*}\right)}{\Omega_{N}(\pi)} \geq \exp (-N R)$, and therefore

$$
P_{\mathrm{e}}>P_{\mathrm{SPB}}\left(N, \theta^{*}, A\right) \text {. }
$$

The use of $\theta^{*}$ instead of the optimal value $\theta_{1}(N, R)$ causes some loss in the tightness of the SP59 bound. However, due to the asymptotic tightness of the bounds on $\frac{\Omega_{N}(\theta)}{\Omega_{N}(\pi)}$, this loss vanishes as $N \rightarrow \infty$. In [36], it was numerically observed that this loss is marginal even for relatively small values of $N R$; it is smaller than $0.01 \mathrm{~dB}$ whenever the dimension of the code is greater than 20 bits, and it is smaller than $0.001 \mathrm{~dB}$ when the dimension exceeds 60 bits.

For large block lengths, the calculation of the SP59 bound becomes difficult in practice due to over and under flows in the floating-point operations. However, [25] presents some asymptotic formulas which give a good estimation of the bound for large enough block lengths. These approximations allow the calculation to be made in the logarithmic domain which eliminates the possibility of floating-point errors.

Theorem 4.2: [25]. Defining

$$
\begin{aligned}
G(\theta) & \triangleq \frac{A \cos \theta+\sqrt{A^{2} \cos ^{2} \theta+4}}{2} \\
E_{L}(\theta) & \triangleq \frac{A^{2}-A G(\theta) \cos \theta-2 \ln (G(\theta) \sin \theta)}{2}
\end{aligned}
$$

then

$$
P_{\mathrm{SPB}}(N, \theta, A) \geq \frac{\sqrt{N-1}}{6 N(A+1)} \exp \left(\frac{3-(A+1)^{2}}{2}\right) \exp \left(-N E_{L}(\theta)\right) .
$$

This lower bound is valid for any block length $N$. However, the ratio of the left and right terms in (104) stays bounded away from one for all $N$.

A rather accurate approximation of $P_{\mathrm{SPB}}(N, \theta, A)$ was provided by Shannon in [25], but without a determined inequality. As a consequence, the following approximation is not a proven theoretical lower bound on the block error probability. For $N>1000$, however, its numerical values become almost identical to those of the exact bound, thus giving a useful estimation for the lower bound.

Proposition 4.1: [25]. Using the notation of Theorem 4.2, if $\theta>\cot ^{-1}(A)$, then

$$
P_{\mathrm{SPB}}(N, \theta, A) \approx \frac{\alpha(\theta) \exp \left(-N E_{L}(\theta)\right)}{\sqrt{N}}
$$

where

$$
\alpha(\theta) \triangleq\left(\sqrt{\pi\left(1+G(\theta)^{2}\right)} \sin \theta\left(A G(\theta) \sin ^{2} \theta-\cos \theta\right)\right)^{-1}
$$

\section{B. A Recent Algorithm for Calculating the 1959 Sphere-Packing Bound}

The SP59 bound has been reviewed in [36, Section 2], and a recursive algorithm has been suggested there to simplify its calculation. This algorithm is presented in the following theorem:

Theorem 4.3 (Recursions for a Simplified Calculation of the SP59 Bound): [36, Theorem 3]. The set of functions $\left\{f_{N}\right\}$ introduced in (95) can be expressed in the alternative form

$$
f_{N}(x)=P_{N}(x)+Q_{N}(x) \exp \left(\frac{x^{2}}{2}\right) \int_{-\infty}^{x} \exp \left(-\frac{t^{2}}{2}\right) \mathrm{d} t, \quad x \in \mathbb{R}, N \in \mathbb{N}
$$


where $P_{N}$ and $Q_{N}$ are polynomials, determined by the same recursive equation for $N \geq 5$

$$
\begin{aligned}
& P_{N}(x)=\frac{2 N-5+x^{2}}{N-1} P_{N-2}(x)-\frac{N-4}{N-1} P_{N-4}(x), \\
& Q_{N}(x)=\frac{2 N-5+x^{2}}{N-1} Q_{N-2}(x)-\frac{N-4}{N-1} Q_{N-4}(x)
\end{aligned}
$$

with the initial polynomials

$$
\begin{aligned}
& P_{1}(x)=0, \quad P_{2}(x)=\sqrt{\frac{2}{\pi}}, \quad P_{3}(x)=\frac{x}{2}, \quad P_{4}(x)=\sqrt{\frac{2}{\pi}} \frac{2+x^{2}}{3}, \\
& Q_{1}(x)=1, \quad Q_{2}(x)=\sqrt{\frac{2}{\pi}} x, \quad Q_{3}(x)=\frac{1+x^{2}}{2}, \quad Q_{4}(x)=\sqrt{\frac{2}{\pi}} \frac{3 x+x^{3}}{3} .
\end{aligned}
$$

By examining the recursive equations for $P_{N}$ and $Q_{N}$ in (108), it is observed that the coefficients of the higher powers of $x$ vanish exponentially as $N$ increases. When performing the calculation using double-precision floating-point numbers, these coefficients cause underflows when $N$ is larger than several hundreds, and are replaced by zeros. Examining the expression for $P_{\mathrm{SPB}}(N, \theta, A)$ in (94), we observe that $f_{N}(x)$ (and therefore the polynomials $P_{N}(x)$ and $\left.Q_{N}(x)\right)$ are evaluated at $x \sim O(\sqrt{N})$. Hence, for large values of $N$, the replacement of the coefficients of the high powers of $x$ by zeros causes a considerable inaccuracy in the calculation of $P_{\mathrm{SPB}}$ in (94).

Considering the integrand in the right side of (94) reveals another difficulty in calculating the SP59 bound for large values on $N$. For large $N$, the term $f_{N}(\sqrt{N} A \cos \phi)$ becomes very large and causes overflows, whereas the term $\sin ^{N-2} \phi$ becomes very small and causes underflows; this causes a " $0 \cdot \infty$ " phenomenon when evaluating the integrand in the right side of (94).

\section{A Log-Domain Approach for Computing the 1959 Sphere-Packing Bound}

In this section, we present a method which facilitates the entire calculation of the integrand in the right side of (94) in the logarithmic domain, thus circumventing the numerical over and under flows which become problematic in the calculation of the SP59 bound for large block lengths. We begin our derivation by representing the set of functions $\left\{f_{N}\right\}$ defined in (95) as sums of exponents.

Proposition 4.2: The set of functions $\left\{f_{N}\right\}$ in (95) can be expressed in the form

$$
f_{N}(x)=\sum_{j=0}^{N-1} \exp (d(N, j, x)), \quad x \in \mathbb{R}, N \in \mathbb{N}
$$

where

$$
\begin{aligned}
d(N, j, x)= & \frac{x^{2}}{2}+\ln \Gamma\left(\frac{N}{2}\right)-\ln \Gamma\left(\frac{j}{2}+1\right)-\ln \Gamma(N-j) \\
& +(N-1-j) \ln (\sqrt{2} x)-\frac{\ln 2}{2} \\
& +\ln \left[1+(-1)^{j} \tilde{\gamma}\left(\frac{x^{2}}{2}, \frac{j+1}{2}\right)\right], \quad \begin{array}{c}
N \in \mathbb{N}, x \in \mathbb{R} \\
\end{array}=0,1 \ldots, N-1
\end{aligned}
$$

and

$$
\begin{aligned}
\Gamma(a) & \triangleq \int_{0}^{\infty} t^{a-1} \exp (-t) \mathrm{d} t, \quad \operatorname{Re}(a)>0 \\
\tilde{\gamma}(x, a) & \triangleq \frac{1}{\Gamma(a)} \int_{0}^{x} t^{a-1} \exp (-t) \mathrm{d} t, \quad x \in \mathbb{R}, \operatorname{Re}(a)>0
\end{aligned}
$$


designate, respectively, the complete and incomplete Gamma functions.

Proof: See Appendix C.

Remark 4.1: Note that the exponents $d(N, j, x)$ in (111) are readily calculated by using standard mathematical functions. The function which calculates the natural logarithm of the Gamma function is implemented in the MATLAB software by gammaln, and in the Mathematica software by LogGamma. The incomplete Gamma function $\tilde{\gamma}$ is implemented in MATLAB by gammainc and in Mathematica by GammaRegularized.

In order to perform the entire calculation of the function $f_{N}$ in the logarithmic domain, we employ the function

$$
\max ^{*}\left(x_{1}, \ldots, x_{m}\right) \triangleq \ln \left(\sum_{i=1}^{m} \exp \left(x_{i}\right)\right), \quad m \in \mathbb{N}, \quad x_{1}, \ldots, x_{m} \in \mathbb{R}
$$

which is commonly used for the implementation of the log-domain BCJR algorithm. The function max* can be calculated in the logarithmic domain using the recursive equation

$$
\begin{aligned}
\max ^{*}\left(x_{1}, \ldots, x_{m+1}\right) & =\max ^{*}\left(\max ^{*}\left(x_{1}, \ldots, x_{m}\right), x_{m+1}\right), \\
m & \in \mathbb{N} \backslash\{1\}, \quad x_{1}, \ldots, x_{m+1} \in \mathbb{R}
\end{aligned}
$$

with the initial value

$$
\max ^{*}\left(x_{1}, x_{2}\right)=\max \left(x_{1}, x_{2}\right)+\ln \left(1+\exp \left(-\left|x_{1}-x_{2}\right|\right)\right) .
$$

Combining Proposition 4.2 and the definition of the function $\max ^{*}$ in (114), we get a method for calculating the set of functions $\left\{f_{N}\right\}$ in the logarithmic domain.

Corollary 4.2: The set of functions $\left\{f_{N}\right\}$ defined in (95) can be rewritten in the form

$$
f_{N}(x)=\exp \left[\max ^{*}(d(N, 0, x), d(N, 1, x), \ldots, d(N, N-1, x))\right]
$$

where $d(N, j, x)$ is introduced in (111).

By combining (94) and (117), one gets the following theorem which provides an efficient algorithm for the calculation of the SP59 bound in the log domain.

Theorem 4.4 (Log domain calculation of the SP59 bound): The term $P_{\mathrm{SPB}}(N, \theta, A)$ in the right side of (101) can be rewritten as

$$
\begin{aligned}
P_{\mathrm{SPB}}(N, \theta, A)= & \int_{\theta}^{\frac{\pi}{2}} \exp \left[\ln (N-1)-\frac{N A^{2}}{2}-\frac{1}{2} \ln (2 \pi)+(N-2) \ln \sin \phi\right. \\
& \left.\quad+\max ^{*}(d(N, 0, \sqrt{N} A \cos \phi), \ldots, d(N, N-1, \sqrt{N} A \cos \phi))\right] \mathrm{d} \phi \\
& +Q(\sqrt{N} A), \quad \forall N \in \mathbb{N}, \theta \in\left[0, \frac{\pi}{2}\right], A \in \mathbb{R}^{+}
\end{aligned}
$$

where the function $d$ is introduced in (111).

\section{Numerical Results FOR SPHERE-PaCking Bounds}

This section presents numerical results to exemplify the improved tightness of the ISP bound for symmetric memoryless channels. For the channel model of an AWGN channel, the ISP and SP59 bounds are compared. All the sphere-packing lower bounds on the decoding error probability are compared to random coding bounds, and also to computer simulations of modern channel codes with practical decoding algorithms. 


\section{A. M-ary PSK Block Coded Modulation Schemes over Fully Interleaved Fading Channels}

Performance bounds of coherently detected M-ary PSK block coded modulation schemes are considered when the transmission takes place over fully interleaved fading channels, and perfect side information of the fading samples is available at the receiver. These bounds are specialized to the fully interleaved Rayleigh-fading channel and the AWGN channel. In the latter case, the ISP bound is compared to the SP59 bound and to some upper bounds on the decoding error probability. For simplicity of notation, we treat the channel inputs and outputs as two-dimensional real vectors. Let $M=2^{p}(p \in \mathbb{N})$ be the size of the PSK constellation, and denote the input to the channel by $\mathbf{X}=\left(X_{1}, X_{2}\right)$ where the possible input values are given by

$$
\mathbf{x}_{k}=\left(\cos \theta_{k}, \sin \theta_{k}\right), \quad \theta_{k} \triangleq \frac{(2 k+1) \pi}{M}, \quad k=0,1, \ldots, M-1 .
$$

We denote the channel output by $(\mathbf{Y}, A)=\left(Y_{1}, Y_{2}, A\right)$ where $A$ is a fading sample whose $p d f$ is given by $p_{A}, \mathbf{Y}=A \mathbf{X}+\mathbf{N}$, and $\mathbf{N}=\left(N_{1}, N_{2}\right)$ is an additive Gaussian random vector with i.i.d. entries of zero-mean and variance $\sigma^{2}$. The channel input, fading and additive noise are statistically independent. Due to the symmetry of the additive noise and the fact that the fading samples are fully known at the receiver, the phase of the fading can be eliminated at the receiver; hence, the fading samples are treated as a non-negative (real) random variables. Due to the channel interleaver, the fading samples are i.i.d. so the channel is memoryless. The conditional $p d f$ of the channel output, given the transmitted symbol $\mathbf{X}_{k}$, is given by

$$
p_{\mathbf{Y}, A \mid \mathbf{X}}\left(\mathbf{y}, a \mid \mathbf{x}_{k}\right)=\frac{p_{A}(a)}{2 \pi \sigma^{2}} \exp \left(-\frac{\left\|\mathbf{y}-a \mathbf{x}_{k}\right\|^{2}}{2 \sigma^{2}}\right), \quad \mathbf{y} \in \mathbb{R}^{2}, a \in \mathbb{R}^{+}
$$

where $\|\cdot\|$ designates the $L_{2}$ norm. Closed-form expressions for the function $\mu_{0}$ and its derivatives are derived in Appendix B.1, and these are used for the calculation of the VF and ISP bounds.

Figure 1 compares the VF bound [36] and the ISP bound derived in Section III. The comparison refers to block codes of length 1024 bits and rate $0.75 \frac{\text { bits }}{\text { channel use }}$ which employ BPSK modulation. Two communication channels are considered: The AWGN channel, which can be viewed as a fading channel where the fading samples are set to 1 (i.e., $p_{A}(a)=\delta(a-1)$ where $\delta$ designates the Dirac delta function), and the fully interleaved Rayleigh-fading channel, where

$$
p_{A}(a)=2 a \exp \left(-a^{2}\right), \quad a \geq 0 .
$$

Further details on BPSK modulated signals transmitted over fully interleaved fading channels, including expressions for the capacity, cutoff rate and various bounds on the decoding error probability, are provided in [23] and references therein. Figure 1 also depicts the capacity limit bound (CLB) for these two channels (calculated from [23, Eq. (2)]). ${ }^{1}$ For a block error probability of $10^{-5}$, the ISP bound provides gains of about 0.19 and $0.33 \mathrm{~dB}$ over the VF bound and gaps of $0.54 \mathrm{~dB}$ and $0.84 \mathrm{~dB}$ to the channel capacity for the AWGN and Rayleigh-fading channels, respectively. Also, for both channels the ISP bound is more informative than the CLB for block error probabilities below $10^{-2}$ while the VF bound requires block error probabilities below $10^{-3}$ to outperform the capacity limit.

\footnotetext{
${ }^{1}$ Although the CLB refers to the asymptotic case where the block length tends to infinity, it is plotted in [36] and here as a reference, in order to examine whether the improvement in the tightness of the ISP is for rates above or below capacity.
} 


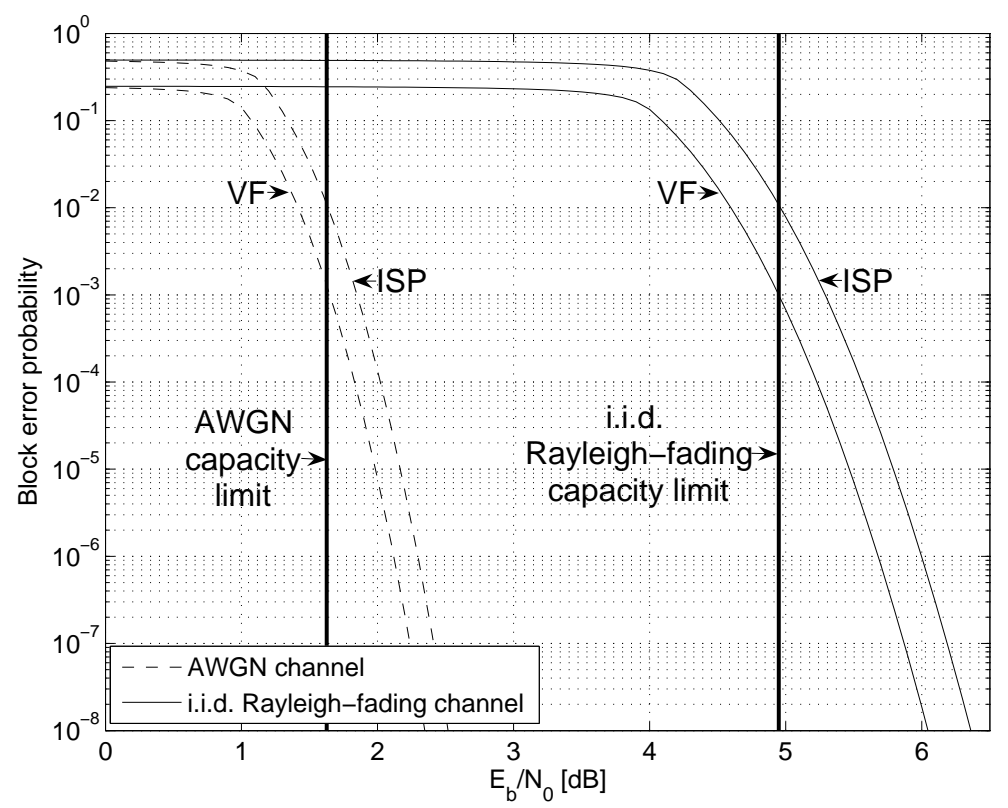

Fig. 1. A comparison between lower bounds on the ML decoding error probability for block codes of length $N=1024$ bits and code rate of $0.75 \frac{\text { bits }}{\text { channel use }}$. This figure refers to BPSK modulated signals whose transmission takes place over fully-interleaved (i.i.d.) Rayleigh-fading and AWGN channels. We compare the Valembois-Fossorier (VF) [36] bound and the improved sphere-packing (ISP) bound derived in Section III.

\section{B. Performance Bounds for M-ary PSK Block Coded Modulation over the AWGN Channel}

A special case of the channel model in Section V-A is the AWGN channel. The closed-form expressions for the function $\mu_{0}$ and its first derivatives are given in Appendix B.2. The SP59 bound [25] provides a lower bound on the decoding error probability for the considered case, since the modulated signals have equal energy and are transmitted over the AWGN channel. In the following, we exemplify the use of these lower bounds. They are also compared to the RCB of Gallager [11], and the tangential-sphere upper bound (TSB) of Poltyrev [20] when applied to random block codes. This serves for studying the tightness of the ISP bound in comparison to other upper and lower bounds. The numerical results shown in this section indicate that the recent variants of the SP67 bound provide an interesting alternative to the SP59 bound which is commonly used in the literature as a measure for the sub-optimality of codes transmitted over the AWGN channel (see, e.g., [9], [15], [18], [24], [30], [36], [38]). Moreover, the advantage of the ISP bound over the VF bound in [36] is exemplified in this section.

Figure 2 compares the SP59 bound [25], the VF bound [36], and the ISP bound in Section III. The comparison refers to block codes of length 500 bits and rate $0.8 \frac{\text { bits }}{\text { channel use }}$ which are BPSK modulated and transmitted over an AWGN channel. The plot also depicts the RCB [11], the TSB ([14], [20]), and the capacity limit bound (CLB). This figure yields that even for relatively short block lengths, the ISP bound outperforms the SP59 bound for block error probabilities below $10^{-1}$ (to be further discussed in this section). For a block error probability of $10^{-5}$, the ISP bound provides gains of about 0.26 and $0.33 \mathrm{~dB}$ over the SP59 and VF bounds, respectively. For fully random block codes with this block length and rate, the TSB provides a tighter upper bound than the RCB; e.g., the gain of the TSB over the RCB is about $0.2 \mathrm{~dB}$ for a block error probability of $10^{-5}$. Note, however, that the RCB is tighter than the TSB for fully random block codes of large enough block lengths, as the latter bound does not reproduce the random-coding 


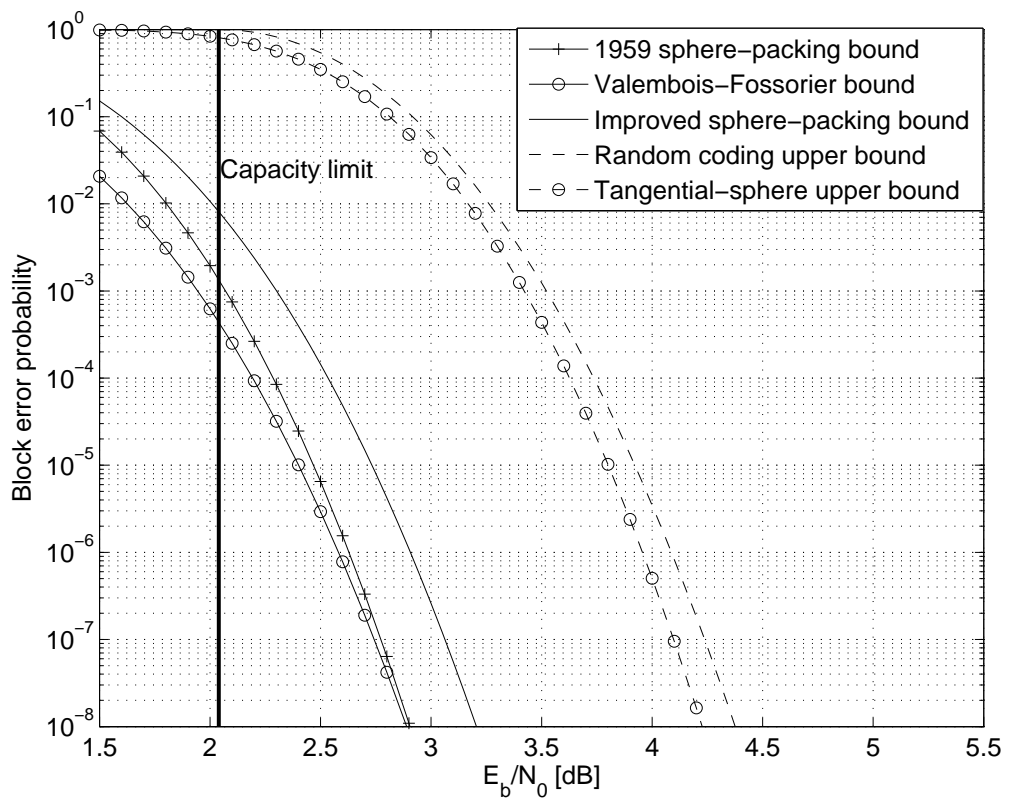

Fig. 2. A comparison between upper and lower bounds on the ML decoding error probability for block codes of length $N=500$ bits and code rate of $0.8 \frac{\text { bits }}{\text { channel use }}$. This figure refers to BPSK modulated signals whose transmission takes place over an AWGN channel. The compared bounds are the 1959 sphere-packing (SP59) bound of Shannon [25], the Valembois-Fossorier (VF) bound [36], the improved sphere-packing (ISP) bound derived in Section III, the random-coding upper bound (RCB) of Gallager [11], and the tangential-sphere upper bound (TSB) [14], [20] when applied to fully random block codes with the above block length and rate.

error exponent for the AWGN channel [20]. However, Figure 2 exemplifies the advantage of the TSB over the RCB when applied to random block codes of relatively short block lengths; this advantage is especially pronounced for low code rates where the gap between the error exponents of these two bounds is marginal (see [24, p. 67]), but it is also shown in Figure 2 for BPSK modulation with a code rate of $0.8 \frac{\text { bits }}{\text { channel use }}$. The gap between the TSB and ISP bounds, as upper and lower bounds respectively, is less than $1.2 \mathrm{~dB}$ for block error probabilities lower than $10^{-1}$. The ISP bound is more informative than the CLB for block error probabilities below $8 \cdot 10^{-3}$ whereas the SP59 and VF bounds require block error probabilities below $1.5 \cdot 10^{-3}$ and $5 \cdot 10^{-4}$, respectively, to outperform the capacity limit.

Figure 3 presents a comparison of the SP59, VF and ISP bounds referring to short block codes which are QPSK modulated and transmitted over the AWGN channel. The plots in Figure 3 also depict the RCB, the TSB and CLB; in these plots, the ISP bound outperforms the SP59 bound for all block error probabilities below $4 \cdot 10^{-1}$ (this result is consistent with the left plot of Figure 7). In the upper plot of Figure 3, which corresponds to a block length of 1024 bits (i.e., 512 QPSK symbols) and a rate of $1.5 \frac{\text { bits }}{\text { channel use }}$, it is shown that the ISP bound provides gains of about 0.25 and $0.37 \mathrm{~dB}$ over the SP59 and VF bounds, respectively, for a block error probability of $10^{-5}$. The gap between the ISP lower bound and the RCB is $0.78 \mathrm{~dB}$ for all block error probabilities lower than $10^{-1}$. In the lower plot of Figure 3 which corresponds to a block length of 300 bits and a rate of $1.8 \frac{\text { bits }}{\text { channel use }}$, the ISP bound significantly improves the SP59 and VF bounds; for a block error probability of $10^{-5}$, the improvement in the tightness of the ISP over the SP59 and VF bounds is 0.8 and $1.13 \mathrm{~dB}$, respectively. Additionally, the ISP bound is more informative than the CLB for block error probabilities below $3 \cdot 10^{-3}$, where the 

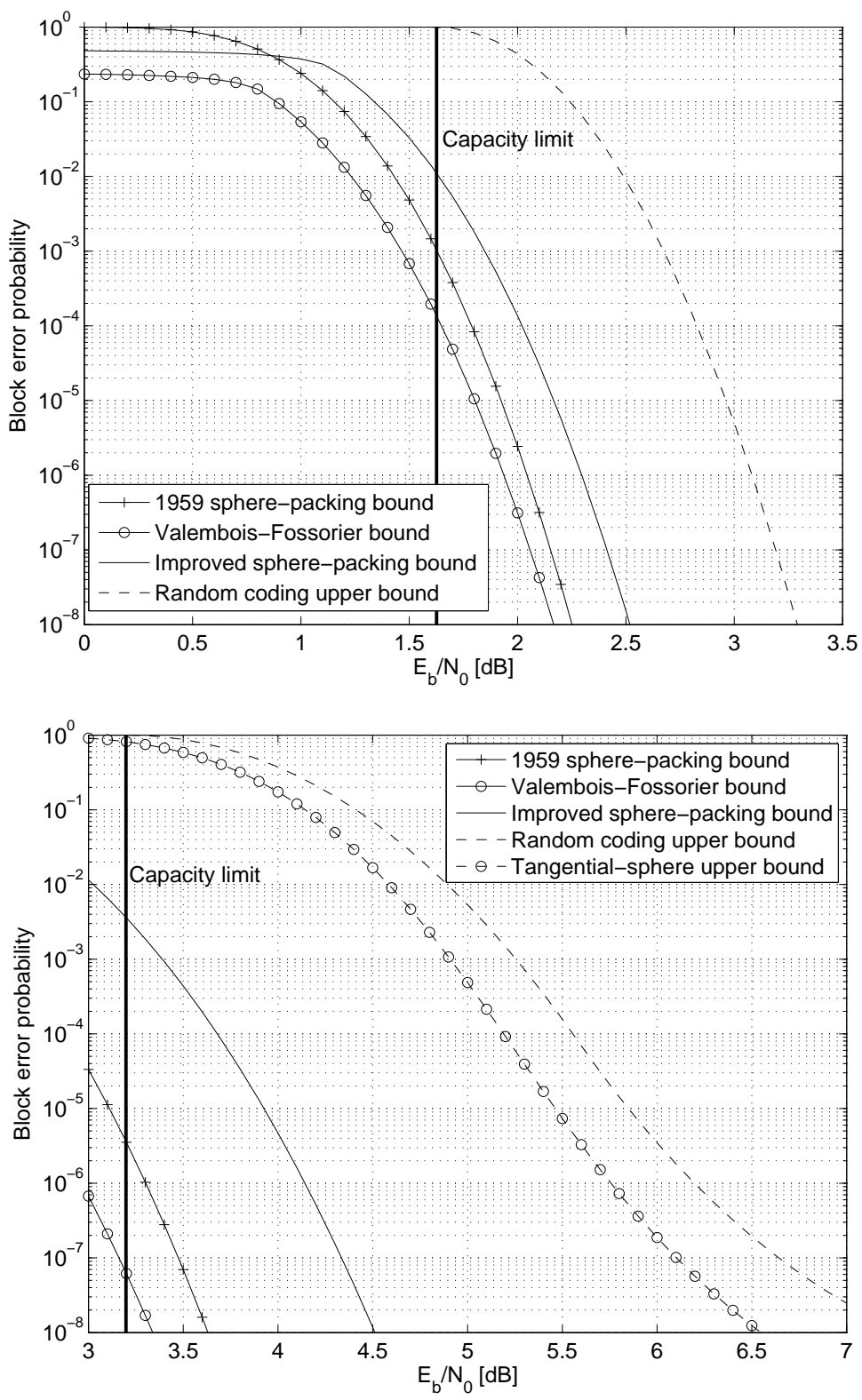

Fig. 3. A comparison between upper and lower bounds on the ML decoding error probability, referring to short block codes which are QPSK modulated and transmitted over the AWGN channel. The compared lower bounds are the 1959 sphere-packing (SP59) bound of Shannon [25], the Valembois-Fossorier (VF) bound [36], and the improved sphere-packing (ISP) bound; the compared upper bounds are the random-coding upper bound (RCB) of Gallager [11] and the tangential-sphere bound (TSB) of Poltyrev [20]. The upper plot refers to block codes of length $N=1024$ which are encoded by 768 information bits (so the rate is $1.5 \frac{\text { bits }}{\text { channel use }}$ ), and the lower plot refers to block codes of length $N=300$ which are encoded by 270 bits whose rate is therefore $1.8 \frac{\text { bits }}{\text { channel use }}$.

SP59 and VF bound outperform the CLB only for block error probabilities below $3 \cdot 10^{-6}$ and $5 \cdot 10^{-8}$, respectively. For fully random block codes of length $N=300$ and rate $1.8 \frac{\text { bits }}{\text { channel use }}$ which are QPSK modulated with Gray's mapping and transmitted over the AWGN channel, the TSB is tighter than the RCB (see the lower plot in Figure 3 and the explanation referring to Figure 2). The gap between the ISP bound and the TSB in this plot is about $1.5 \mathrm{~dB}$ for a block 
error probability of $10^{-5}$ (as compared to gaps of $2.3 \mathrm{~dB}(2.63 \mathrm{~dB})$ between the TSB and the SP59 (VF) bound).
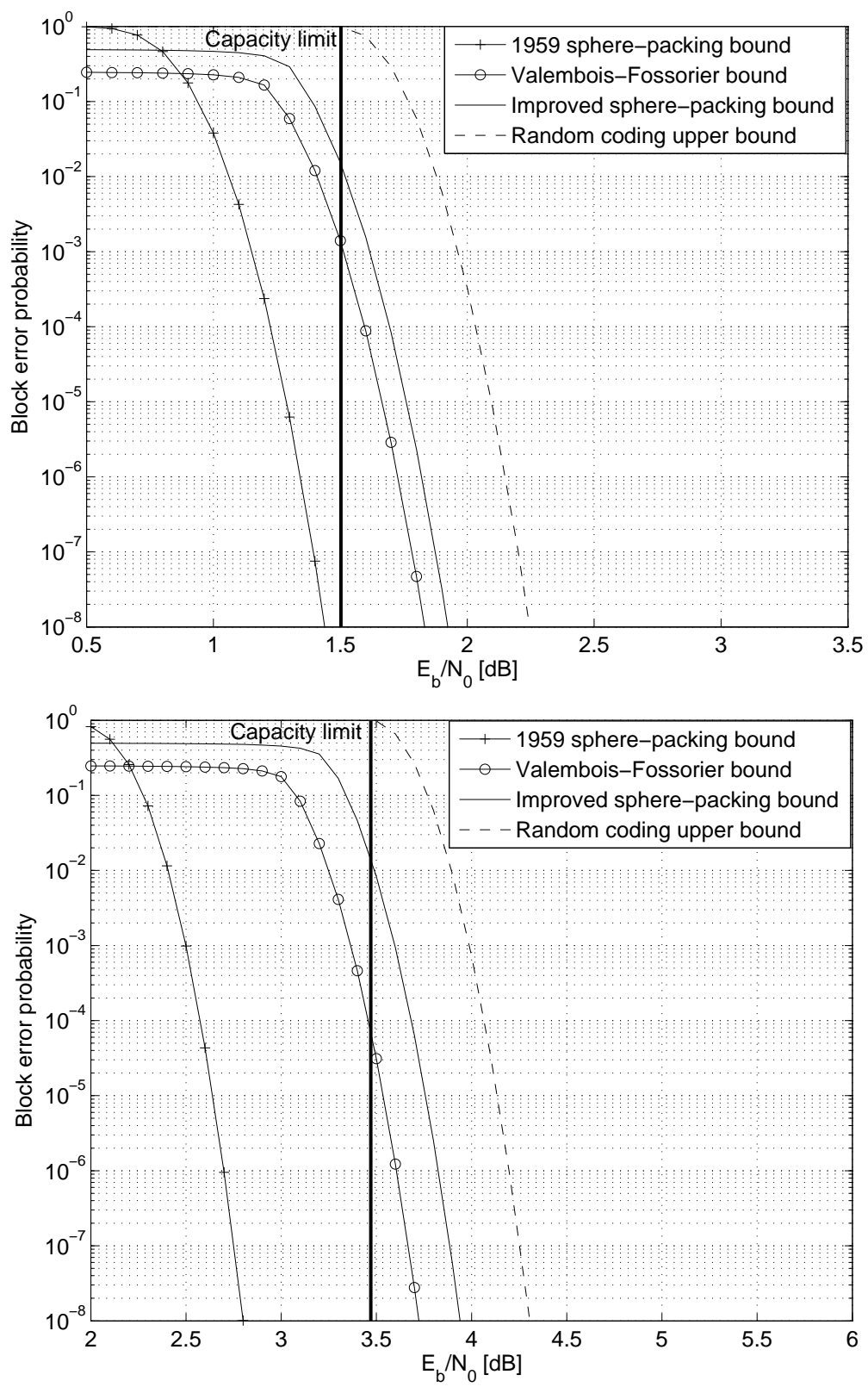

Fig. 4. A comparison of upper and lower bounds on the ML decoding error probability for block codes of length $N=5580$ bits and information block length of 4092 bits. This figure refers to QPSK (upper plot) and 8-PSK (lower plot) modulated signals whose transmission takes place over an AWGN channel; the rates in this case are 1.467 and $2.200 \frac{\text { bits }}{\text { channel use }}$, respectively. The compared bounds are the 1959 sphere-packing (SP59) bound of Shannon [25], the Valembois-Fossorier (VF) bound [36], the improved sphere-packing (ISP) bound, and the random-coding upper bound (RCB) of Gallager [11].

Figure 4 presents a comparison of the bounds for codes of block length 5580 bits and 4092 information bits, where both QPSK (upper plot) and 8-PSK (lower plot) constellations are considered. The modulated signals correspond to 2790 and 1860 symbols, respectively, so the 
code rates for these constellations are 1.467 and 2.2 bits per channel use, respectively. For both constellations, the two considered SP67-based bounds (i.e., the VF and ISP bounds) outperform the SP59 for all block error probabilities below $2 \cdot 10^{-1}$; the ISP bound provides gains of 0.1 and $0.22 \mathrm{~dB}$ over the VF bound for the QPSK and 8-PSK constellations, respectively. For both modulations, the gap between the ISP lower bound and the RCB upper bound does not exceed $0.4 \mathrm{~dB}$. In [6], Divsalar and Dolinar designed codes with the considered parameters by using concatenated Hamming and accumulate codes. They also present computer simulations of the performance of these codes under iterative decoding, when the transmission takes place over the AWGN channel and several common modulation schemes are applied. For a block error probability of $10^{-4}$, the gap between the simulated performance of these codes under iterative decoding, and the ISP lower bound, which gives an ultimate lower bound on the block error probability of optimally designed codes under ML decoding, is approximately $1.4 \mathrm{~dB}$ for QPSK and 1.6 dB for 8-PSK signaling. This provides an indication on the performance of codes defined on graphs and their iterative decoding algorithms, especially in light of the feasible complexity of the decoding algorithm which is linear in the block length. To conclude, it is reflected from the results plotted in Figure 4 that a gap of about $1.5 \mathrm{~dB}$ between the ISP lower bound and the performance of the iteratively decoded codes in [6] is mainly due to the imperfectness of these codes and their sub-optimal iterative decoding algorithm; this conclusion follows in light of the fact that for random codes of the same block length and rate, the gap between the ISP bound and the RCB is reduced to less than $0.4 \mathrm{~dB}$.

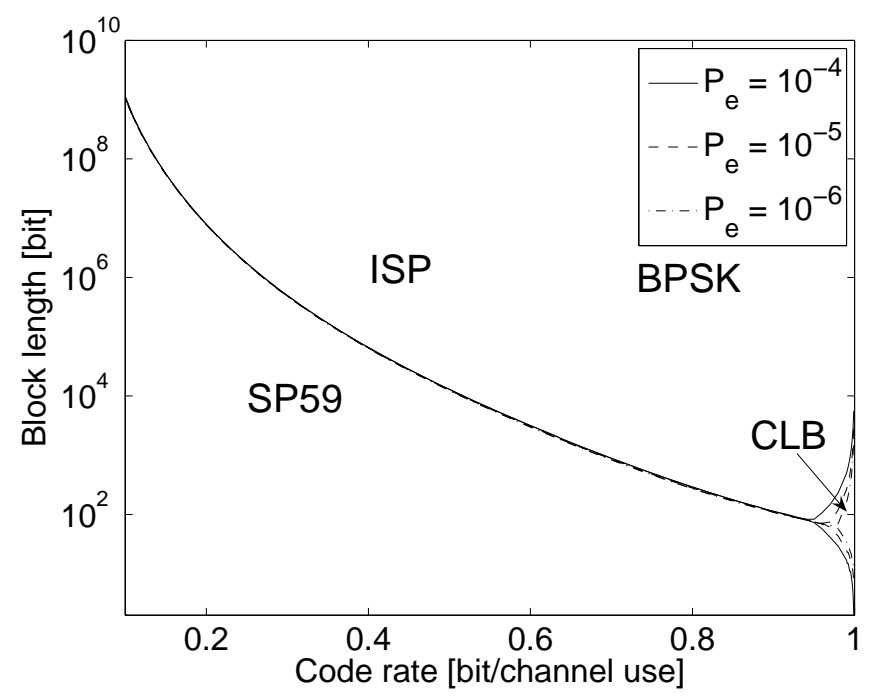

Fig. 5. Regions in the two-dimensional space of code rate and block length, where a bound is better than the two others for three different targets of block error probability $\left(P_{\mathrm{e}}\right)$. The figure compares the tightness of the 1959 sphere-packing (SP59) bound of Shannon [25], the improved sphere-packing (ISP) bound, and the capacity-limit bound (CLB). The plot refers to BPSK modulated signals whose transmission takes place over the AWGN channel, and the considered code rates lie in the range between 0.1 and $1 \frac{\text { bits }}{\text { channel use }}$.

While it was shown in Section III that the ISP bound is uniformly tighter than the VF bound (which in turn is uniformly tighter than the SP67 bound [26]), no such relations are shown between the SP59 bound and the recent improvements on the SP67 bound (i.e., the VF and ISP bounds). Figure 5 presents regions of code rates and block lengths for which the ISP bound outperforms the SP59 bound and the CLB; it refers to BPSK modulated signals transmitted over the AWGN channel and considers block error probabilities of $10^{-4}, 10^{-5}$ and $10^{-6}$. It is 

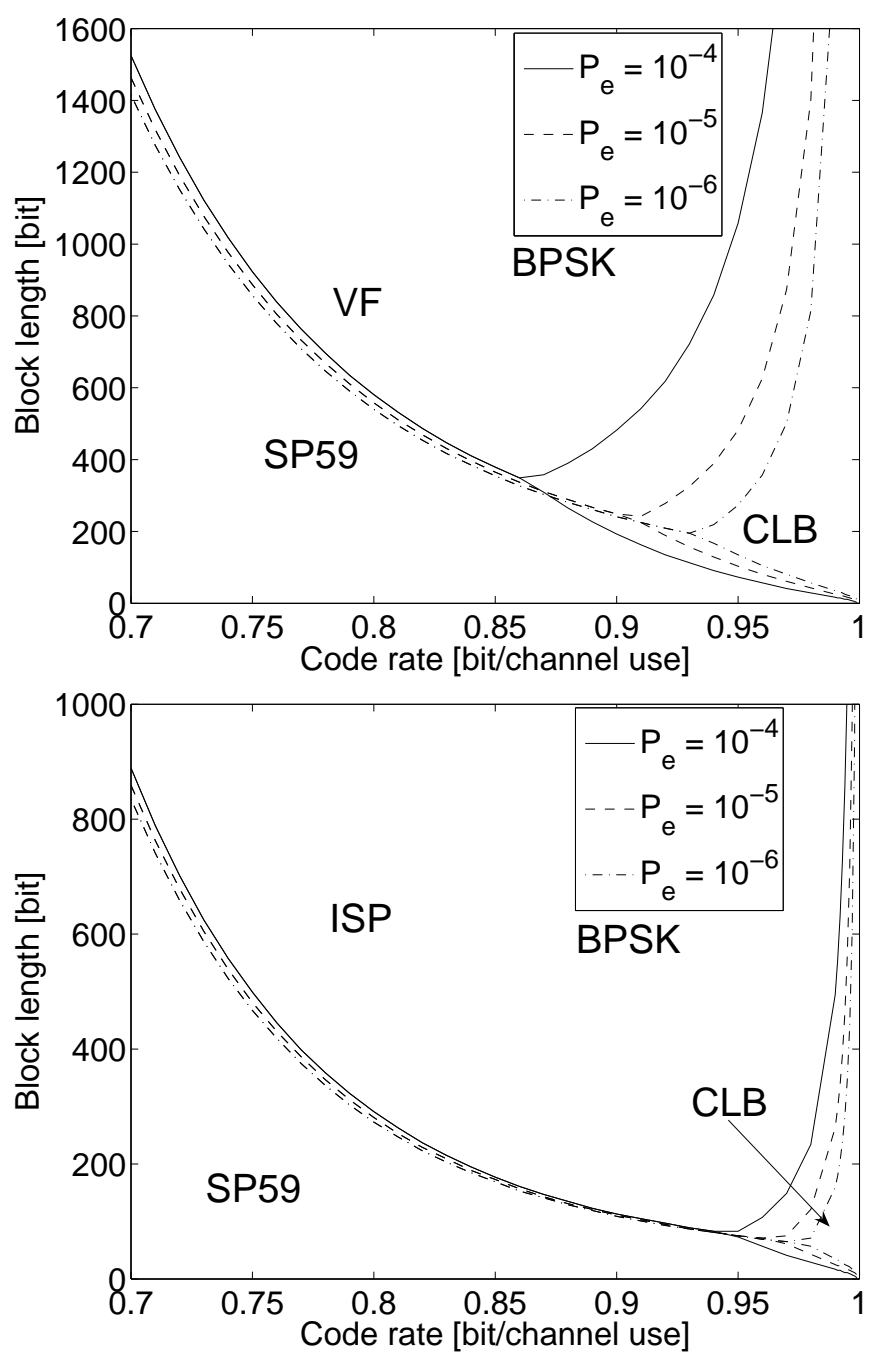

Fig. 6. Regions in the two-dimensional space of code rate and block length, where a bound is better than the two others for three different targets of block error probability $\left(P_{\mathrm{e}}\right)$. The figure compares the tightness of the 1959 sphere-packing (SP59) bound of Shannon [25], the capacity-limit bound (CLB), and the Valembois-Fossorier (VF) bound [36] (left plot) or the improved sphere-packing (ISP) bound in Section III (right plot). The plots refer to BPSK modulated signals whose transmission takes place over the AWGN channel, and the considered code rates lie in the range between 0.70 and $1 \frac{\text { bits }}{\text { channel use }}$.

reflected from this figure that for any rate $0<R<1$, there exists a block length $N=N(R)$ such that the ISP bound outperforms the SP59 bound for block lengths larger than $N(R)$; the same property also holds for the VF bound, but the value of $N(R)$ depends on the considered SP67-based bound, and it becomes significantly larger in the comparison of the VF and SP59 bounds. It is also observed that the value $N(R)$ is monotonically decreasing with $R$, and it approaches infinity as we let $R$ tend to zero. An intuitive explanation for this behavior can be given by considering the capacity limits of the binary-input and the energy-constrained AWGN channels. For any value $0 \leq C<1$, denote by $\frac{E_{\mathrm{b}, 1}(C)}{N_{0}}$ and $\frac{E_{\mathrm{b}, 2}(C)}{N_{0}}$ the values of $\frac{E_{\mathrm{b}}}{N_{0}}$ required to achieve a channel capacity of $C$ bits per channel use for the binary-input and the energyconstrained AWGN channels, respectively (note that in the latter case, the input distribution which 

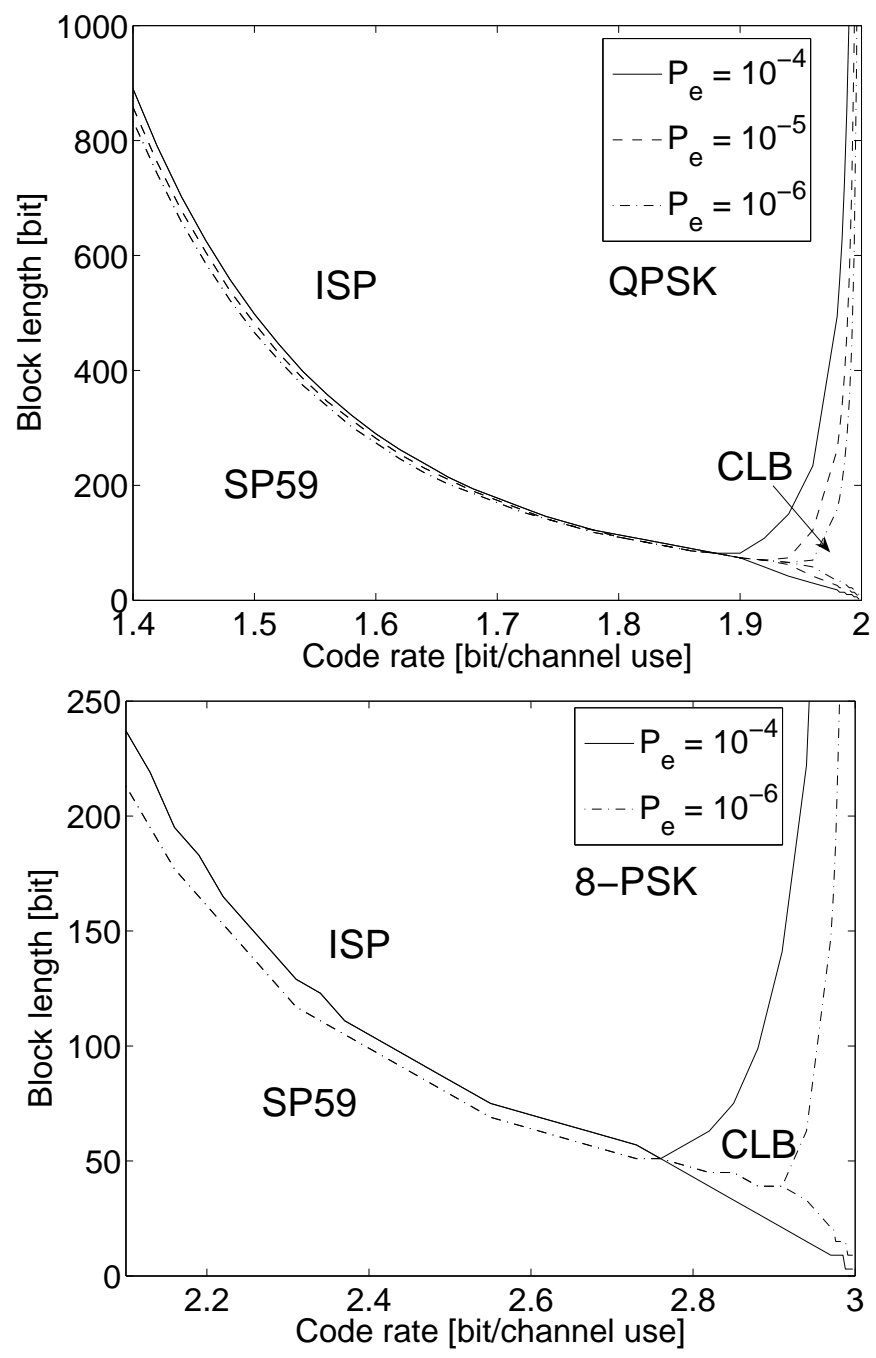

Fig. 7. Regions in the two-dimensional space of code rate and block length, where a bound is better than the two others for different targets of block error probability $\left(P_{\mathrm{e}}\right)$. The figure compares the tightness of the 1959 sphere-packing (SP59) bound of Shannon [25], the improved sphere-packing (ISP) bound, and the capacity-limit bound (CLB). The plots refer to QPSK (left plot) and 8-PSK (right plot) modulated signals whose transmission takes place over the AWGN channel; the considered code rates lie in the range between 1.4 and $2 \frac{\text { bits }}{\text { channel use }}$ for the QPSK modulated signals and between 2.1 and $3 \frac{\text { bits }}{\text { channel use }}$ for the 8-PSK modulated signals.

achieves capacity is also Gaussian). For any $0 \leq C<1$, clearly $\frac{E_{\mathrm{b}, 1}(C)}{N_{0}} \geq \frac{E_{\mathrm{b}, 2}(C)}{N_{0}}$; however, the difference between these values is monotonically increasing with the capacity $C$, and, on the other hand, this difference approaches zero as we let $C$ tend to zero. Since the SP59 bound only constrains the signals to be of equal energy, it gives a measure of performance for the energyconstrained AWGN channel, where the SP67-based bounds consider the actual modulation and therefore refer to the binary-input AWGN channel. As the code rates become higher, the difference in the ultimate performance between the two channels is larger, and therefore the SP67-based bounding techniques outperform the SP59 bound for smaller block lengths. For low code rates, the difference in the ultimate performance between the channels is reduced, and the SP59 outperforms the SP67-based bounding techniques even for larger block lengths due to the 
superiority of the former bound which is specifically tailored for the AWGN channel. Figure 6 presents the regions of code rates and block lengths for which the VF bound (left plot) and the ISP bound (right plot) outperform the CLB and the SP59 bound when the signals are BPSK modulated and transmitted over the AWGN channel; block error probabilities of $10^{-4}, 10^{-5}$ and $10^{-6}$ are examined. This figure is focused on high code rates, where the performance of the SP67-based bounds and their advantage over the SP59 bound is most appealing. From Figure 6, for a code rate of 0.75 bits per channel use and a block error probability of $10^{-6}$, the VF bound becomes tighter than the SP59 for block lengths exceeding 850 bits while the ISP bound reduces this value to 450 bits; moreover, by increasing the rate to 0.8 bits per channel use, the respective minimal block lengths reduce to 550 and 280 bits for the VF and ISP bounds, respectively. Fig 7 shows the regions of code rates and block lengths where the ISP outperforms the CLB and SP59 bounds for QPSK (left plot) and 8-PSK (right plot) modulations. Comparing the right plot of Figure 6 which refers to BPSK modulation with the left plot of Figure 7 which refers to QPSK modulation, one can see that the two graphs are identical (when accounting for the doubling of the rate which is due to the use of both real and imaginary dimensions in the QPSK modulation). This is due to the fact that QPSK modulation poses no additional constraints on the channel and in fact, the real and imaginary planes can be serialized and decoded as in BPSK modulation. However, this property does not hold when replacing the ISP bound by the VF bound; this is due to the fact that the VF bound considers a fixed composition subcode of the original code and the increased size of the alphabet causes a greater loss in the rate for QPSK modulation. When comparing the two plots of Figure 7, it is evident that the minimal value of the block length for which the ISP bound becomes better than the SP59 bound decreases as the size of the input alphabet is increased (when the rate is measured in units of information bits per code bit). An intuitive justification for this phenomenon is attributed to the fact that referring to the constellation points of the M-ary PSK modulation, the mutual information between the code symbols in each dimension of the QPSK modulation is zero, while as the spectral efficiency of the PSK modulation is increased, the mutual information between the real and imaginary parts of each signal point is increased; thus, as the spectral efficiency is increased, this poses a stronger constraint on the possible positioning of the equal-energy signal points on the $N$-dimensional sphere. This intuition suggests an explanation for the advantage of the ISP bound over the SP59 bound, which holds even for smaller block lengths as the spectral efficiency is increased (recall that the SP59 bound does not take into account the modulation scheme). This effect is expected to be more subtle for the VF bound since a larger size of the input alphabet decreases the rate for which the error exponent is evaluated (see (31)).

\section{Performance Bounds for the Binary Erasure Channel}

In recent years, several families of code ensembles defined on graphs have been constructed and demonstrated to achieve the capacity of the BEC under iterative decoding with low complexity (see, e.g., [17], [19] and [28]). These low-complexity and capacity-achieving ensembles for the BEC motivate a study of the performance of iteratively decoded codes defined on graphs for moderate block lengths (see, e.g., [34]). In Figure 8, we compare the ISP bound with the exact block error probability of the ensemble of fully random and binary linear block codes under ML decoding where the transmission takes place over the BEC (see [5, Eq. (3.2)]). This figure refers to codes of rate 0.75 bits per channel use and various block lengths. It can be observed that for a block length of 1024 bits, the difference in the channel erasure probability for which the RCB and the ISP bound achieve a block error probability of $10^{-5}$ is 0.035 while for a block length of 16384 bits, this gap is decreased to 0.009 . This yields that the ISP bound is reasonably tight, 


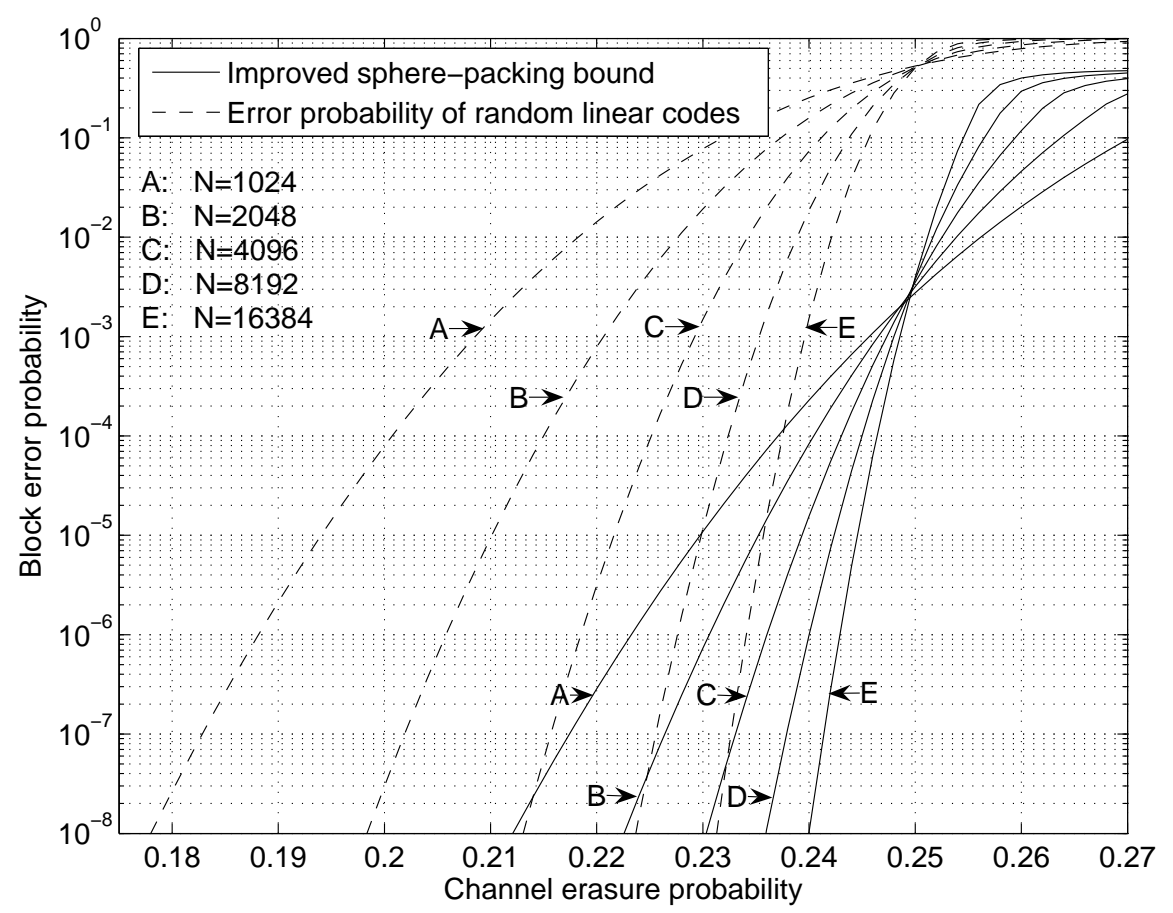

Fig. 8. A comparison of the improved sphere-packing (ISP) lower bound from Section III and the exact decoding error probability of random binary linear block codes under ML decoding where the transmission takes place over the BEC (see [5, Eq. (3.2)]). The code rate examined is $0.75 \frac{\text { bits }}{\text { channel use }}$ and the block lengths are $N=1024,2048,4096,8192$ and 16384 bits.

and also suggests that this bound can be used in order to assess the imperfectness of turbo-like codes even for moderate block lengths.

\section{Minimal Block Length as a Function of Performance}

In a wide range of applications, the system designer needs to design a communication system which fulfills several requirements on the available bandwidth, acceptable delay for transmitting and processing the data while maintaining a certain fidelity criterion in reconstructing the data (e.g., the block error probability needs to be below a certain threshold). In this setting, one wishes to design a code which satisfies the delay constraint (i.e., the block length is limited) while adhering to the required performance over the given channel. By fixing the communication channel model, code rate (which is related to the bandwidth expansion caused by the errorcorrecting code) and the block error probability, sphere-packing bounds are transformed into lower bounds on the minimal block length required to achieve the desired block error probability at a certain gap to capacity using an arbitrary block code and decoding algorithm. Similarly, by fixing these parameters, upper bounds on the error probability of random codes under ML decoding are transformed into upper bounds on the block length required for ML decoded random codes to achieve a desired block error probability on a given communication channel.

In this section, we consider some practically decodable codes taken from some recent papers ([1], [7], [8], [29], [31], [35]). We examine the gap between channel capacity and the $\frac{E_{\mathrm{b}}}{N_{0}}$ for which they achieve a required block error probability as a function of the block length of these codes. The performance of these specific codes under their practical decoding algorithms is compared to the sphere-packing bounds and also to upper bounds on the error probability of 
random block codes; these bounds serve here as lower and upper bounds, respectively, on the block length required to achieve a given block error probability and code rate on a given channel using an optimal block code and decoding algorithm. Comparing the performance of specific codes and decoding algorithms to the information-theoretic limitations provided by the spherepacking bounds, enables one to deduce how far in terms of delay is a practical system from the fundamental limitations of information theory.

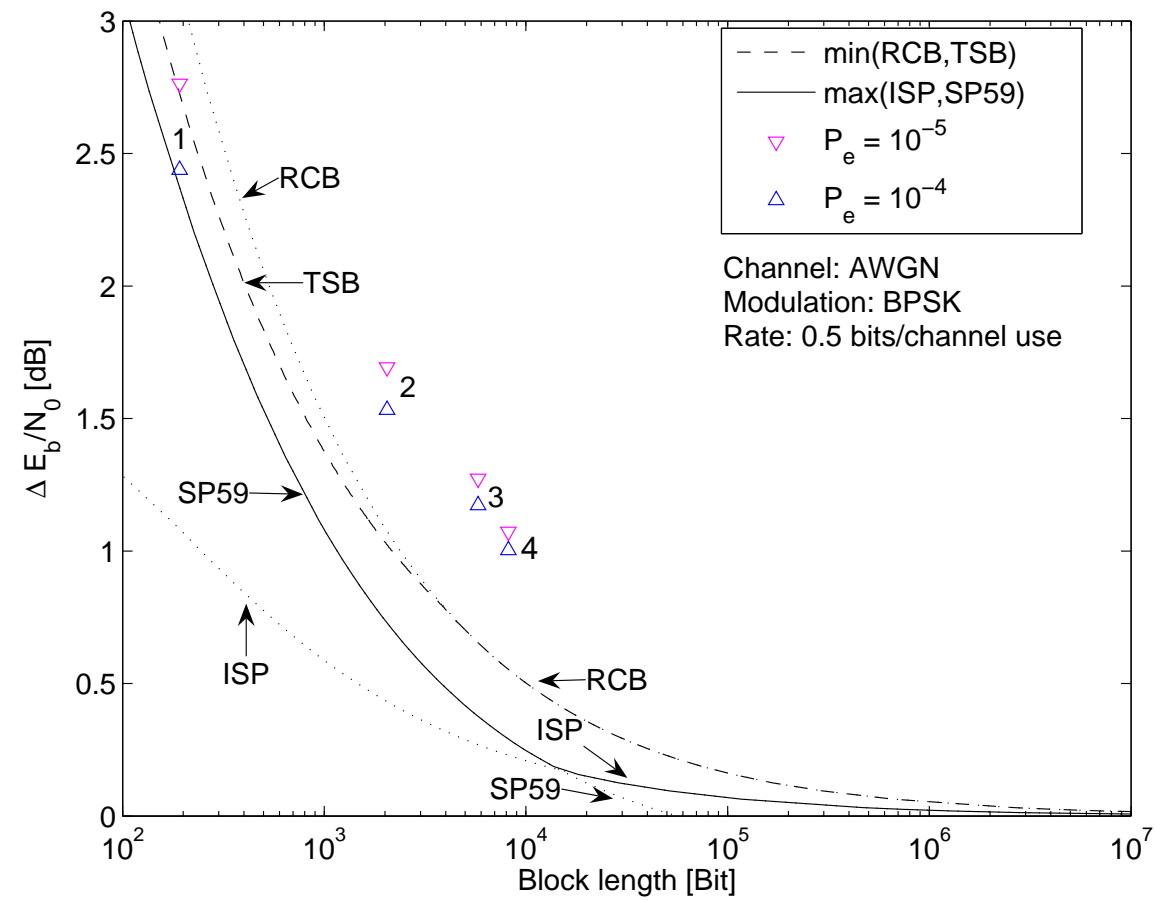

Fig. 9. This figure refers to the tradeoff between the block length and the gap to capacity of error-correcting codes which are BPSK modulated and transmitted over an AWGN channel. The horizontal axis refers to the block length of the codes, and the vertical axis refers to the gap, measured in decibels, between the channel capacity and the energy per bit to spectral noise density $\left(\frac{E_{\mathrm{b}}}{N_{0}}\right)$ which is required to obtain a block error probability $P_{\mathrm{e}}$ (set either to $10^{-4}$ or $10^{-5}$ ). The considered rate of all the codes is one-half bit per channel use. The minimal gap to capacity which is required for achieving a block error probability of $10^{-5}$ is depicted via bounds: the upper bound is calculated using the random-coding bound (RCB) of Gallager [11] and the tangential-sphere bound (TSB) of Poltyrev [20] applied to fully random and binary block codes, and the lower bound on this minimal block length is calculated via the 1959 sphere-packing (SP59) bound of Shannon [25] and the improved sphere-packing (ISP) bound introduced in Section III. In addition to bounds, this tradeoff between the block length (delay) and gap to capacity, is shown for some efficiently decodable error-correcting codes; the codes are taken from [35] (code 1), [8] (codes 2 and 4) and [7] (code 3).

Figure 9 considers some block codes of rate $\frac{1}{2}$ bits per channel use which are BPSK modulated and transmitted over the AWGN channel. The plot depicts the gap to capacity in $\mathrm{dB}$ for which these codes achieve block error probabilities of $10^{-4}$ and $10^{-5}$ under their practical decoding algorithms as a function of their block length. As a reference, this figure also plots lower bounds on the block length which stem from the SP59 and ISP bounds, and upper bounds on the block length of fully random binary block codes which are based on the RCB [11] and the TSB of Poltyrev [20]; these bounds refer to a block error probability of $10^{-5}$. For large enough block lengths, the RCB provides a tighter upper bound on the achievable gap to capacity than the TSB; this is expected since the error exponent of the TSB is slightly looser than the random-coding 
error exponent (see [32, upper plot of Fig. 3]). However, for small to moderate block lengths (i.e., for block lengths below approximately 5000 bits according to Figure 9), the TSB provides a tighter upper bound on the achievable gap as compared to the RCB. The improvement of the TSB over the RCB closes the gap between the upper and lower bounds on the achievable gap to capacity for small to moderate block lengths (where the lower bound is obtained via the SP59 bound which is tighter than the ISP bound for the considered range of block lengths). As for particularly efficient block codes, the code labeled 1 in Figure 9 is a block code of length 192 bits which is decoded using a near-ML decoder by applying 'box and match' decoding techniques [35]. It is observed that this code outperforms RCB for ML decoded random codes with the same block length and code rate, and almost coincides with the upper bound obtained via the TSB. It is also observed that this code achieves a block error probability of $10^{-5}$ at a gap to capacity of $2.76 \mathrm{~dB}$ while the SP59 bound gives that the block length required to achieve this performance is lower bounded by 133 bits (so the bound is very informative). The codes labeled 2, 3 and 4 are prototype-based LDPC codes of lengths 2048, 5176 and 8192 bits, respectively (codes 2 and 4 are taken from [8] and code 3 is taken from [7]). These codes achieve under iterative decoding a block error probability of $10^{-5}$ at gaps to capacity of 1.70 , 1.27 and $1.07 \mathrm{~dB}$, respectively. In terms of block length, the gap between the performance of these codes under iterative decoding and the SP59 lower bound on the block length required to achieve a block error probability of $10^{-5}$ at these channel conditions is less than one order of magnitude. It is also noted that throughout the range of block lengths depicted in Figure 9, the gap between the lower bound on the block length of optimal codes which stems from the better of the two sphere-packing bounds and the upper bound on the block length of random codes is less than one order of magnitude. This exemplifies the tightness of the sphere-packing bounds when used as lower bounds on the block lengths of optimal codes.

Figure 10 considers some LDPC codes of rate 0.88 bits per channel use which are BPSK modulated and transmitted over the AWGN channel. The gap to capacity in $\mathrm{dB}$ for which these codes achieve block error probabilities of $10^{-4}$ and $10^{-5}$ under iterative decoding is plotted as a function of block length. As in Figure 9, the figure uses upper and lower bounds on the achievable gap to capacity in terms of the block length: for this (relatively high) code rate and the considered range of block lengths, the ISP bound is uniformly tighter than the SP59 bound (so only the ISP bound is depicted in this figure, and the SP59 bound is omitted). The upper bounds on the required block lengths for achieving a target block error probability in terms of the achievable gap to capacity are obtained via the RCB and the TSB when it is applied to the ensemble of fully random block codes. The upper and lower bounds refer to a block error probability of $10^{-5}$. Similarly to Figure 9, the RCB is advantageous over the TSB for block codes of short to moderate block lengths; in this case, the advantage of the RCB over the TSB occurs for block lengths above approximately 1000 bits (instead of 5000 bits, as was the case in Fig. 9 for code rate of 0.5 bit per channel use). This shows that for short block lengths, the TSB is tighter than the RCB; however, since by increasing the code rate, the error exponent of the TSB becomes less tight as compared to the error exponent of the RCB (see the upper and lower plots of [32, Fig. 3] which refers to code rates of 0.5 and 0.9 bits per channel use), the asymptotic advantage of the RCB over the TSB is more pronounced, and the former bound is tighter than the latter already for shorter block lengths. The tradeoff between the gap to capacity (in terms of $\frac{E_{\mathrm{b}}}{N_{0}}$ ) versus the block length is depicted in Figure 10 for some efficient error-correcting codes, in order to compare their practical performance and delay to the information-theoretic bounds (similarly to Fig. 9). For the examined block error probabilities (of $10^{-4}$ and $10^{-5}$ ), the depicted codes require a gap to capacity of between 0.63 and $1.9 \mathrm{~dB}$. For 


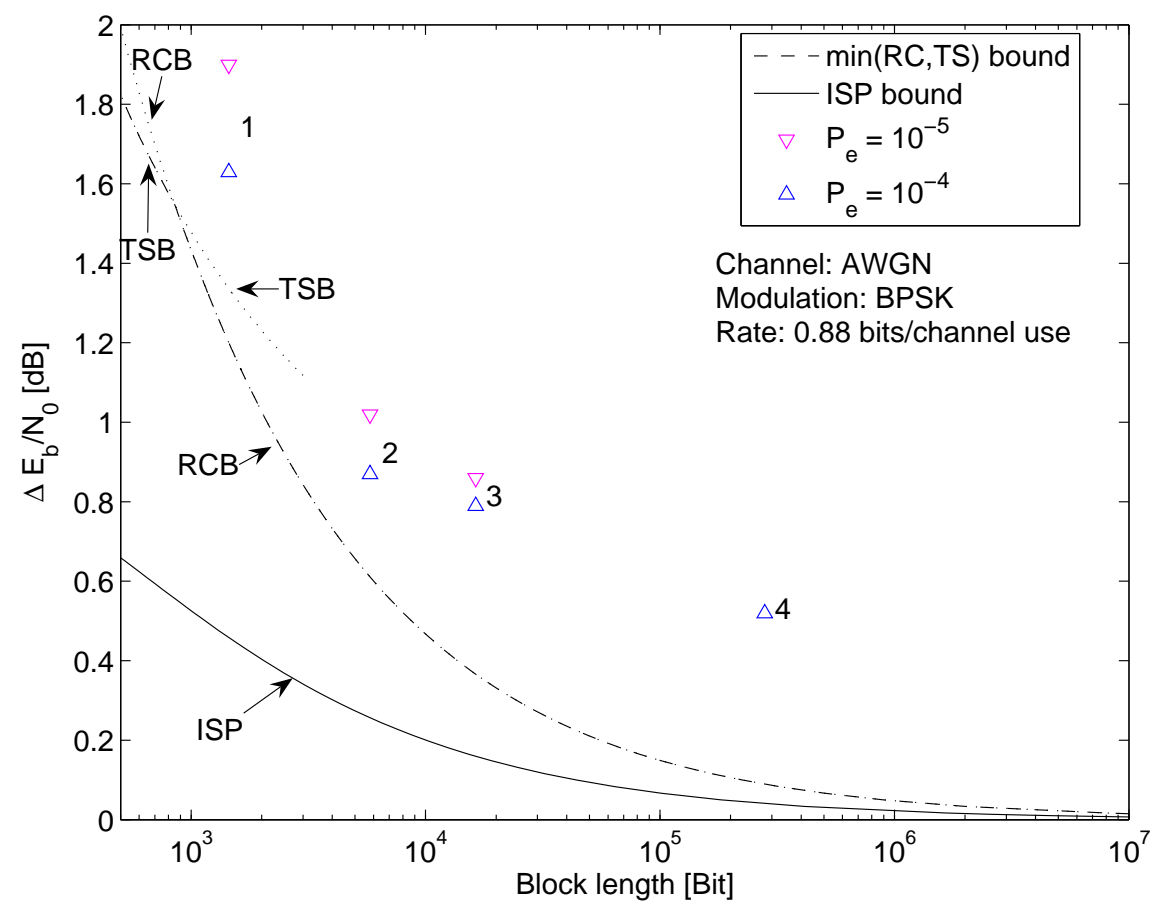

Fig. 10. This figure refers to the tradeoff between the block length and the gap to capacity of error-correcting codes which are BPSK modulated and transmitted over an AWGN channel. The horizontal axis refers to the block length of the codes, and the vertical axis refers to the gap, measured in decibels, between the channel capacity and the energy per bit to spectral noise density $\left(\frac{E_{\mathrm{b}}}{N_{0}}\right)$ which is required to obtain a block error probability $P_{\mathrm{e}}$ (set either to $10^{-4}$ or $10^{-5}$ ). The considered rate of all the codes is 0.88 bit per channel use. The minimal gap to capacity which is required for achieving a block error probability of $10^{-5}$ is depicted via bounds: the upper bound is calculated using the random-coding bound (RCB) [11] and the tangential-sphere bound (TSB) [20] applied to fully random and binary block codes, and the lower bound on this minimal block length is calculated via the improved sphere-packing (ISP) bound introduced in Section III (which is better than the 1959 sphere-packing (SP59) bound [25] for the considered code rate and the range of block lengths which is depicted in the horizontal line). In addition to bounds, this tradeoff between the block length (delay) and gap to capacity, is shown for some efficiently decodable error-correcting codes; the codes labeled by 1, 2, 3 and 4 are taken from [1], [7], [29] and [31], respectively.

this range of $\frac{E_{\mathrm{b}}}{N_{0}}$, the lower bound on the block lengths which is derived from the ISP bound is looser than the one given by the SP59 bound. However, both bounds are not very informative in this range. For cases where the gap to capacity is below $0.5 \mathrm{~dB}$, the difference between the lower bound on the block length of optimal codes which stems from the ISP bound and the upper bound on the block length of random codes is less than one order of magnitude. Code number 1 is an LDPC of length 1448 bits whose construction of is based on balanced incomplete block designs [1]. This code achieves a block error probability of $10^{-5}$ at a gap to capacity of $1.9 \mathrm{~dB}$ while the RCB shows that the block length which is required to achieve this performance using random codes is upper bounded by 600 bits. The code labeled 2 is a prototype-based LDPC code of length 5176 bits which is taken from [7]. Code number 3 is a quasi-cyclic LDPC code of length 16362 bits taken from [29]. These code achieve under iterative decoding a block error probability of $10^{-5}$ at gaps to capacity of 1.02 and $0.86 \mathrm{~dB}$, respectively. In terms of block length, the gap between the performance of these codes under iterative decoding and the upper bound on the block length of random codes which achieve a block error probability of $10^{-5}$ under the same channel conditions is less than one order of magnitude. The code labeled 4 is a 
finite-geometry LDPC code of length 279552 bits which is taken from [31]. For this code we only have the gap to capacity required to achieve a block error probability of $10^{-4}$, however, it is clear that the difference in block length from the RCB becomes quite large as the gap to capacity is reduced.

By fixing the block length and considering the gap in $\Delta E_{\mathrm{b}} / N_{0}$ between the performance of the specific codes and the sphere-packing bounds in Figures 9 and 10, it is observed that the codes considered in these plots exhibit gaps of $0.2-0.8 \mathrm{~dB}$ with respect to the information-theoretic limitation provided by the sphere-packing bounds (with the exception of code 1 in Figure 10 which exhibits a gap of about $1.25 \mathrm{~dB}$ ). In this respect we also mention that some high rate turbo-product codes with moderate block lengths (see [4]) exhibit a gap of $0.75-0.95 \mathrm{~dB}$ with respect to the information-theoretic limitation provided by the ISP bound. Based on numerical results in [33] for the ensemble of uniformly interleaved $(1144,1000)$ turbo-block codes whose components are random systematic, binary and linear block codes, the gap in $\frac{E_{\mathrm{b}}}{N_{0}}$ between the ISP lower bound and an upper bound under ML decoding is $0.9 \mathrm{~dB}$ for a block error probability of $10^{-7}$. These results exemplify the strength of the sphere-packing bounds for assessing the theoretical limitations of block codes and the power of iteratively decoded codes (see also [9], [15], [16], [24], [36]).

\section{SUMMARY}

This paper presents an improved sphere-packing (ISP) bound for finite-length block codes whose transmission takes place over symmetric memoryless channels. The improved tightness of the bound is especially pronounced for codes of short to moderate block lengths, and some of its applications are exemplified in this paper. The derivation of the ISP bound was stimulated by the remarkable performance and feasible complexity of turbo-like codes with short to moderate block lengths. We were motivated by recent improvements on the sphere-packing bound of [26] for finite block lengths, as suggested by Valembois and Fossorier [36].

We first review the classical sphere-packing bounds, i.e., the 1959 sphere-packing bound (SP59) derived by Shannon for equal-energy signals transmitted over the Gaussian channel [25], and the 1967 sphere-packing (SP67) bound derived by Shannon, Gallager and Berlekamp for discrete memoryless channels [26]. The ISP bound, introduced in Section III, is uniformly tighter than the classical SP67 bound [26] and the bound in [36].

We apply the ISP bound to various memoryless symmetric channels. The tightness of the ISP bound is exemplified by comparing it with upper and lower bounds on the ML decoding error probability and also with reported computer simulations of turbo-like codes under iterative decoding.

This paper also presents a new numerical algorithm which performs the entire calculation of the SP59 bound in the logarithmic domain, thus facilitating the exact calculation of the SP59 bound for all block lengths without the need for asymptotic approximations. It is shown that the ISP bound suggests an interesting alternative to the SP59 bound, where the latter is specialized for the AWGN channel.

In a wide range of applications, one wishes to design a block code which satisfies a known delay constraint (i.e., the block length is limited) while adhering to a required performance over a given channel model. By fixing the communication channel model, code rate and the block error probability, sphere-packing bounds are transformed into lower bounds on the minimal block length required to achieve the target block error probability at a certain gap to capacity when an arbitrary block code and decoding algorithm are used. Comparing the performance of specific codes and decoding algorithms to the information-theoretic limitations provided by the spherepacking bounds, enables one to deduce how far in terms of delay is a practical system from 
the fundamental limitations of information theory. Further details on the comparison between practically decodable codes and the sphere-packing bounds are found in Section V-D.

The ISP bound is especially attractive for block codes of short to moderate block lengths, and its advantage is especially pronounced for high rate codes. Its improvement over the SP67 bound and the bound in [36, Theorem 7] also becomes more significant as the input alphabet of the considered modulation is increased.

\section{Acknowledgment}

The authors are grateful to Prof. Amos Lapidoth for raising the question which stimulated the discussion in Section V-D. The authors also would like to acknowledge the anonymous reviewers for their constructive reviews which led to a clarification in the presentation (Remark 3.5), and the associate editor, Prof. Kingo Kobayashi, for handling the paper.

\section{APPENDICES}

\section{ApPendix A: Proof of Lemma 3.1}

We consider a symmetric DMC with input alphabet $\mathcal{K}=\{0, \ldots, K-1\}$, output alphabet $\mathcal{J}=\{0, \ldots J-1\}$ (where $J, K \in \mathbb{N}$ ) and a transition probability function $P(\cdot \mid \cdot)$. Let $\left\{g_{k}\right\}_{k=0}^{K}$ be the set of unitary functions which satisfy the conditions (41) and (42) in Definition 3.2. To prove Lemma 3.1, we start with a discussion on the distribution $\mathbf{q}_{s}$ which satisfies (67).

a) On the input distribution $\mathbf{q}_{s}$ for symmetric DMCs:

Lemma A.1: For symmetric DMCs and an arbitrary value of $s \in(0,1)$, the uniform distribution $q_{k, s}=\frac{1}{K}$ for $k \in \mathcal{K}$ satisfies (67) with equality.

Proof: To prove the lemma, it is required to show that

$$
\sum_{j=0}^{J-1}\left\{P(j \mid k)^{1-s}\left(\sum_{k^{\prime}=0}^{K-1} \frac{1}{K} P\left(j \mid k^{\prime}\right)^{1-s}\right)^{\frac{s}{1-s}}\right\}=\sum_{j=0}^{J-1}\left(\sum_{k^{\prime}=0}^{K-1} \frac{1}{K} P\left(j \mid k^{\prime}\right)^{1-s}\right)^{\frac{1}{1-s}}, \quad \forall k \in \mathcal{K} .
$$

Let us consider some $k \in \mathcal{K}$. Examining the left side of (A.1) gives

$$
\begin{aligned}
& \sum_{j=0}^{J-1}\left\{P(j \mid k)^{1-s}\left(\sum_{k^{\prime}=0}^{K-1} \frac{1}{K} P\left(j \mid k^{\prime}\right)^{1-s}\right)^{\frac{s}{1-s}}\right\} \\
& =K \sum_{j=0}^{J-1}\left\{\frac{1}{K} P(j \mid k)^{1-s}\left(\sum_{k^{\prime}=0}^{K-1} \frac{1}{K} P\left(j \mid k^{\prime}\right)^{1-s}\right)^{\frac{s}{1-s}}\right\} \\
& \stackrel{(a)}{=} \sum_{\widetilde{k}=0}^{K-1} \sum_{j=0}^{J-1}\left\{\frac{1}{K} P(j \mid k)^{1-s}\left(\sum_{k^{\prime}=0}^{K-1} \frac{1}{K} P\left(j \mid k^{\prime}\right)^{1-s}\right)^{\frac{s}{1-s}}\right\} \\
& \stackrel{(b)}{=} \sum_{\widetilde{k}=0}^{K-1} \sum_{j=0}^{J-1}\left\{\frac{1}{K} P\left(g_{\widetilde{k}}(j) \mid k\right)^{1-s}\left(\sum_{k^{\prime}=0}^{K-1} \frac{1}{K} P\left(g_{\widetilde{k}}(j) \mid k^{\prime}\right)^{1-s}\right)^{\frac{s}{1-s}}\right\}
\end{aligned}
$$

where $(a)$ holds by summing over a dummy variable $\widetilde{k} \in \mathcal{K}$ instead of the multiplication by $K$ in the previous line, and $(b)$ holds since $g_{\widetilde{k}}$ is unitary for all $\widetilde{k} \in \mathcal{K}$ (see (37) where the integral 
is replaced here by a sum). For all $j \in \mathcal{J}$ and $\widetilde{k} \in \mathcal{K}$, the symmetry properties in (41) - (43) give

$$
\begin{aligned}
P\left(g_{\widetilde{k}}(j) \mid k\right) & \stackrel{(a)}{=} P\left(\left(g_{k}^{-1} \circ g_{\widetilde{k}}\right)(j) \mid 0\right) \\
& \stackrel{(b)}{=} P\left(g_{(\widetilde{k}-k) \bmod K}(j) \mid 0\right) \\
& \stackrel{(c)}{=} P(j \mid(k-\widetilde{k}) \bmod K)
\end{aligned}
$$

where $(a)$ follows from (41), (b) relies on (42), and (c) follows from (41) and (43). Similarly, for all $j, \widetilde{k} \in\{0,1, \ldots, K-1\}$

$$
\begin{aligned}
\sum_{k^{\prime}=0}^{K-1} \frac{1}{K} P\left(g_{\widetilde{k}}(j) \mid k^{\prime}\right)^{1-s} & \stackrel{(a)}{=} \sum_{k^{\prime}=0}^{K-1} \frac{1}{K} P\left(j \mid\left(k^{\prime}-\widetilde{k}\right) \bmod K\right)^{1-s} \\
& \stackrel{(b)}{=} \sum_{k^{\prime}=0}^{K-1} \frac{1}{K} P\left(j \mid k^{\prime}\right)^{1-s}
\end{aligned}
$$

where $(a)$ relies on (A.3) and $(b)$ holds since when $k^{\prime}$ takes all the values in $\{0,1, \ldots, K-1\}$, so does $\left(k^{\prime}-\widetilde{k}\right) \bmod K$. Substituting (A.3) and (A.4) in (A.2) gives

$$
\begin{aligned}
& \sum_{j=0}^{J-1}\left\{P(j \mid k)^{1-s}\left(\sum_{k^{\prime}=0}^{K-1} \frac{1}{K} P\left(j \mid k^{\prime}\right)^{1-s}\right)^{\frac{s}{1-s}}\right\} \\
& =\sum_{\widetilde{k}=0}^{K-1} \sum_{j=0}^{J-1}\left\{\frac{1}{K} P(j \mid(k-\widetilde{k}) \bmod K)^{1-s}\left(\sum_{k^{\prime}=0}^{K-1} \frac{1}{K} P\left(j \mid k^{\prime}\right)^{1-s}\right)^{\frac{s}{1-s}}\right\} \\
& =\sum_{j=0}^{J-1}\left\{\left(\sum_{\widetilde{k}=0}^{K-1} \frac{1}{K} P(j \mid(k-\widetilde{k}) \bmod K)^{1-s}\right)\left(\sum_{k^{\prime}=0}^{K-1} \frac{1}{K} P\left(j \mid k^{\prime}\right)^{1-s}\right)^{\frac{s}{1-s}}\right\} \\
& \stackrel{(a)}{=} \sum_{j=0}^{J-1}\left\{\left(\sum_{\widetilde{k}=0}^{K-1} \frac{1}{K} P(j \mid \widetilde{k})^{1-s}\right)\left(\sum_{k^{\prime}=0}^{K-1} \frac{1}{K} P\left(j \mid k^{\prime}\right)^{1-s}\right)^{\frac{s}{1-s}}\right\} \\
& =\sum_{j=0}^{J-1}\left(\sum_{k^{\prime}=0}^{K-1} \frac{1}{K} P\left(j \mid k^{\prime}\right)^{1-s}\right)^{\frac{1}{1-s}}
\end{aligned}
$$

where equality $(a)$ holds since the above index $\widetilde{k}$ takes all the values in $\{0,1, \ldots, K-1\}$, and so does the index $k^{\prime} \triangleq(k-\widetilde{k}) \bmod K$.

We now turn to explore how the symmetry of the channel and the input distribution $\mathbf{q}_{s}$ induce a symmetry on the probability tilting measure $f_{s}$.

b) On the symmetry of the tilting measure $f_{s}$ for strictly symmetric DMCs:

Lemma A.2: For all $s \in(0,1), k \in \mathcal{K}$ and $j \in \mathcal{J}$, the tilting measure $f_{s}$ in (69) satisfies

$$
f_{s}(j)=f_{s}\left(g_{k}(j)\right) \text {. }
$$

Proof: Examining the definition of $f_{s}$ in (69), it can be observed that it suffices to show that

$$
\alpha_{j, s}=\alpha_{g_{k}(j), s}, \quad \forall s \in(0,1), k \in \mathcal{K}, j \in \mathcal{J}
$$


where $\alpha_{j, s}$ is given in (68). Note that for the uniform input distribution where $q_{k, s}=\frac{1}{K}$ for all $k \in \mathcal{K}$, inequality (67) and (68) holds with equality (see Lemma A.1). From (A.4), equality (A.7) follows while referring to this uniform input distribution.

Having established some symmetry properties of $\mathbf{q}_{s}$ and $f_{s}$, we are ready to prove equalities (70) $-(72)$.

c) On the independence of $\mu_{k}$ and its two derivatives from $k$ : As we have shown, the uniform distribution $\mathbf{q}_{s}$ satisfies (67) in equality for all inputs, so

$$
\begin{aligned}
\mu_{k}(s) & =\ln \left(\sum_{j} P(j \mid k)^{1-s} f_{s}(j)^{s}\right) \\
& \stackrel{(a)}{=} \ln \left(\sum_{j} P(j \mid k)^{1-s}\left(\alpha_{j, s}\right)^{\frac{s}{1-s}}\right)-s \ln \left(\sum_{j}\left(\alpha_{j, s}\right)^{\frac{1}{1-s}}\right) \\
& \stackrel{(b)}{=}(1-s) \ln \left(\sum_{j}\left(\alpha_{j, s}\right)^{\frac{1}{1-s}}\right) \\
& \stackrel{(c)}{=}(1-s) \ln \left(\sum_{j}\left[\sum_{k} q_{k, s} P(j \mid k)^{1-s}\right]^{\frac{1}{1-s}}\right)
\end{aligned}
$$

where $(a)$ follows from the choice of $f_{s}$ in (68) and (69), (b) follows from Lemma A.1 and (68), and (c) follows from (68). Under the setting $s=\frac{\rho}{1+\rho}$, since the conditions on $\mathbf{q}_{s}$ in (67) are identical to the conditions on the input distribution $\mathbf{q}=\mathbf{q}_{s}$ which maximizes $E_{0}\left(\frac{s}{1-s}, \mathbf{q}\right)$ as stated in [11, Theorem 4], then

$$
\begin{aligned}
\mu_{k}\left(s, f_{s}\right) & =(1-s) \ln \left(\sum_{j}\left[\sum_{k} q_{k, s} P(j \mid k)^{\frac{1}{1+\frac{s}{1-s}}}\right]^{1+\frac{s}{1-s}}\right) \\
& =-(1-s) E_{0}\left(\frac{s}{1-s}, \mathbf{q}_{s}\right) \\
& =-(1-s) E_{0}\left(\frac{s}{1-s}\right), \quad 0<s<1
\end{aligned}
$$

where $E_{0}$ is given in (25). This proves (70).

We now turn to prove the independence of the first two derivatives of $\mu_{k}$ with respect to $s$ from $k \in \mathcal{K}$.

Remark A.1: Note that the partial derivative of $\mu_{k}(s)$ with respect to $s$ is performed while holding $f=f_{s}$ constant.

As is shown in [26],

$$
\begin{aligned}
& \mu^{\prime}(s)=\mathbb{E}_{Q_{s}}(D(j)) \\
& \mu^{\prime \prime}(s)=\operatorname{Var}_{Q_{s}}(D(j))
\end{aligned}
$$

where

$$
D(j) \triangleq \ln \left(\frac{P_{2}(j)}{P_{1}(j)}\right), \quad Q_{s}(j) \triangleq \frac{P_{1}(j)^{1-s} P_{2}(j)^{s}}{\sum_{j^{\prime}} P_{1}\left(j^{\prime}\right)^{1-s} P_{2}\left(j^{\prime}\right)^{s}} .
$$

For every $k \in \mathcal{K}, P_{1}$ and $P_{2}$ used in $\mu_{k}$ are defined to be $P(\cdot \mid k)$ and $f_{s}$, respectively. Hence, for all $k \in \mathcal{K}$

$$
\begin{aligned}
\mu_{k}^{\prime}\left(s, f_{s}\right) & =\mathbb{E}_{Q_{k, s}}\left(D_{k, s}(j)\right) \\
\mu_{k}^{\prime \prime}\left(s, f_{s}\right) & =\operatorname{Var}_{Q_{k, s}}\left(D_{k, s}(j)\right)
\end{aligned}
$$


where

$$
\begin{aligned}
D_{k, s}(j) & \triangleq \ln \left(\frac{f_{s}(j)}{P(j \mid k)}\right) \\
Q_{k, s}(j) \triangleq & \frac{P(j \mid k)^{1-s} f_{s}(j)^{s}}{\sum_{j^{\prime}=0}^{J-1} P\left(j^{\prime} \mid k\right)^{1-s} f_{s}\left(j^{\prime}\right)^{s}} .
\end{aligned}
$$

Applying (41) and Lemma A.2, we get that for all $k \in \mathcal{K}$

$$
\begin{aligned}
\sum_{j^{\prime}=0}^{J-1} P\left(j^{\prime} \mid k\right)^{1-s} f_{s}\left(j^{\prime}\right)^{s} & \stackrel{(a)}{=} \sum_{j^{\prime}=0}^{J-1} P\left(g_{k}^{-1}\left(j^{\prime}\right) \mid 0\right)^{1-s} f_{s}\left(g_{k}^{-1}\left(j^{\prime}\right)\right)^{s} \\
& \stackrel{(b)}{=} \sum_{j^{\prime}=0}^{J-1} P\left(j^{\prime} \mid 0\right)^{1-s} f_{s}\left(j^{\prime}\right)^{s}
\end{aligned}
$$

where $(a)$ follows from (41) and Lemma A.2, and $(b)$ follows since $g_{k}^{-1}$ is unitary. Substituting (A.13) in (A.12) gives

$$
\begin{aligned}
Q_{k, s}(j) & =\frac{P(j \mid k)^{1-s} f_{s}(j)^{s}}{\sum_{j^{\prime}=0}^{J-1} P\left(j^{\prime} \mid k\right)^{1-s} f_{s}\left(j^{\prime}\right)^{s}} \\
& \stackrel{(a)}{=} \frac{P\left(g_{k}^{-1}(j) \mid 0\right)^{1-s} f_{s}\left(g_{k}^{-1}(j)\right)^{s}}{\sum_{j^{\prime}=0}^{J-1} P\left(j^{\prime} \mid 0\right)^{1-s} f_{s}\left(j^{\prime}\right)^{s}} \\
& \stackrel{(b)}{=} Q_{0, s}\left(g_{k}^{-1}(j)\right)
\end{aligned}
$$

where (a) follows from (41), (43), (69), (A.6) and (A.13), and (b) relies on the definition of $Q_{k, s}$ in (A.12). Similarly,

$$
\begin{aligned}
D_{k, s}(j) & =\ln \left(\frac{f_{s}(j)}{P(j \mid k)}\right) \\
& \stackrel{(a)}{=} \ln \left(\frac{f_{s}\left(g_{k}^{-1}(j)\right)}{P\left(g_{k}^{-1}(j) \mid 0\right)}\right) \\
& \stackrel{(b)}{=} D_{0, s}\left(g_{k}^{-1}(j)\right)
\end{aligned}
$$

where $(a)$ follows from (41), (43) and (A.6), and (b) relies on the definition of $D_{k, s}$ in (A.11). Using (A.14) and (A.15), we finally get for all $k \in \mathcal{K}$

$$
\begin{aligned}
\mu_{k}^{\prime}(s) & =\mathbb{E}_{Q_{k, s}}\left(D_{k, s}(j)\right) \\
& =\sum_{j=0}^{J-1} Q_{k, s}(j) D_{k, s}(j) \\
& =\sum_{j=0}^{J-1} Q_{0, s}\left(g_{k}^{-1}(j)\right) D_{0, s}\left(g_{k}^{-1}(j)\right)
\end{aligned}
$$




$$
\begin{aligned}
& \stackrel{(a)}{=} \sum_{j=0}^{J-1} Q_{0, s}(j) D_{0, s}(j) \\
& =\mu_{0}^{\prime}(s)
\end{aligned}
$$

and

$$
\begin{aligned}
\mu_{k}^{\prime \prime}(s) & =\operatorname{Var}_{Q_{k, s}}\left(D_{k, s}(j)\right) \\
& =\sum_{j=0}^{J-1} Q_{k, s}(j) D_{k, s}^{2}(j)-\mu_{k}^{\prime}(s)^{2} \\
& =\sum_{j=0}^{J-1} Q_{0, s}\left(g_{k}^{-1}(j)\right)\left(D_{0, s}\left(g_{k}^{-1}(j)\right)\right)^{2}-\mu_{0}^{\prime}(s)^{2} \\
& \stackrel{(b)}{=} \sum_{j=0}^{J-1} Q_{0, s}(j)\left(D_{0, s}(j)\right)^{2}-\mu_{0}^{\prime}(s)^{2} \\
& =\mu_{0}^{\prime \prime}(s)
\end{aligned}
$$

where $(a)$ and $(b)$ follow since $g_{k}^{-1}$ is unitary for all $k \in \mathcal{K}$. This completes the proof of Lemma 3.1.

Remark A.2: Equalities (70)-(72) hold for arbitrary symmetric memoryless channels. For a general output alphabet $\mathcal{J} \subseteq \mathbb{R}^{d}$, the proof of these properties follows the same lines as the proof here with the exception that the sums over $\mathcal{J}$ are replaced by integrals. As in Definition 3.1, if the projection of $\mathcal{J}$ over some of the $d$ dimensions is countable, the integration over these dimensions is turned into a sum.

\section{APPENDiX B: CALCUlation OF THE FunCTION $\mu_{0}$ IN (66) FOR SOME SyMmetRiC CHANNELS}

This appendix presents some technical calculations which yield the expressions for the function $\mu_{0}$ defined in (66) and its first two derivatives with respect to $s$ (while holding $f_{s}$ fixed in the calculation of the partial derivatives of $\mu$ with respect to $s$, as required in [26]). The examined cases are M-ary PSK modulated signals transmitted over fully interleaved fading channels, with the AWGN channel as a special case, and binary block codes transmitted over the BEC. These expressions serve for the application of the VF bound in [36] and the ISP bound derived in Section III to block codes transmitted over these channels.

\section{A. M-ary PSK Modulated Signal over Fully Interleaved Fading Channels with Perfect CSI}

For M-ary PSK modulated signals transmitted over a fully interleaved fading channel, the channel output is $\mathcal{J}=\mathbb{R}^{2} \times \mathbb{R}^{+}$, where the first two coordinates refer to the vector $\mathbf{Y}$ and the third refers to the fading coefficient $A$. In the case of a continuous output alphabet, the sums in (A.8) are replaced by integrals, and the transition probabilities are replaced by transition probability density functions. To simplify the presentation, for all $s \in(0,1), \mathbf{y} \in \mathbb{R}^{2}$ and $a \in \mathbb{R}^{+}$we define

$$
\kappa_{s}(\mathbf{y}, a) \triangleq\left(\frac{1}{M} \sum_{k=0}^{M-1} e^{\frac{(1-s) a\left\langle\mathbf{y}, \mathbf{x}_{k}-\mathbf{x}_{0}\right\rangle}{\sigma^{2}}}\right)^{\frac{1}{1-s}} .
$$


This expression will be used throughout the following calculations.

Due to the symmetry of the channel, we get from Lemma A.1 that the distribution $\mathbf{q}_{s}$ which satisfies (67) is uniform. Hence, we get by substituting (120) into (A.8) that

$$
\mu_{0}(s)=(1-s) \ln \left(\iint_{\mathbb{R}^{2}} \int_{0}^{\infty} \frac{p_{A}(a)}{2 \pi \sigma^{2}} e^{-\frac{\left\|\mathbf{y}-a \mathbf{x}_{0}\right\|^{2}}{2 \sigma^{2}}} \zeta_{s}(\mathbf{y}, a) \mathrm{d} a \mathrm{~d} \mathbf{y}\right)
$$

where

$$
\zeta_{s}(\mathbf{y}, a) \triangleq\left(\frac{1}{M} \sum_{k=0}^{M-1} e^{-\frac{(1-s)\left(\left\|\mathbf{y}-a \mathbf{x}_{k}\right\|^{2}-\left\|\mathbf{y}-a \mathbf{x}_{0}\right\|^{2}\right)}{2 \sigma^{2}}}\right)^{\frac{1}{1-s}}
$$

Since $\left\|x_{k}\right\|^{2}=1$ for all $k \in\{0,1, \ldots, M-1\}$ we have

$$
\left\|\mathbf{y}-a \mathbf{x}_{k}\right\|^{2}-\left\|\mathbf{y}-a \mathbf{x}_{0}\right\|^{2}=-2 a\left\langle\mathbf{y}, \mathbf{x}_{k}-\mathbf{x}_{0}\right\rangle
$$

and so $\mu_{0}$ can be rewritten in the form

$$
\mu_{0}(s)=(1-s) \ln (\theta(s))
$$

where

$$
\theta(s) \triangleq \iint_{\mathbb{R}^{2}} \int_{0}^{\infty} \frac{p_{A}(a)}{2 \pi \sigma^{2}} e^{-\frac{\left\|\mathbf{y}-a \mathbf{x}_{0}\right\|^{2}}{2 \sigma^{2}}} \kappa_{s}(\mathbf{y}, a) \mathrm{d} a \mathrm{~d} \mathbf{y} .
$$

and $\kappa_{s}(\mathbf{y}, a)$ is defined in (B.1).

We now turn to calculate the derivative of $\mu_{0}$ with respect to $s$ while holding $f=f_{s}$ constant. Substituting (120) into the definition of $f_{s}$ in (69), we get that $f_{s}$ is given by

$$
\begin{aligned}
f_{s}(\mathbf{y}, a) & =\frac{\left(\sum_{k=0}^{M-1} \frac{1}{M}\left(\frac{p_{A}(a)}{2 \pi \sigma^{2}}\right)^{1-s} e^{-\frac{(1-s)\left\|\mathbf{y}-a \mathbf{x}_{k}\right\|^{2}}{2 \sigma^{2}}}\right)^{\frac{1}{1-s}}}{\iint_{\mathbb{R}^{2}} \int_{0}^{\infty}\left(\sum_{k=0}^{M-1} \frac{1}{M}\left(\frac{p_{A}\left(a^{\prime}\right)}{2 \pi \sigma^{2}}\right)^{1-s} e^{-\frac{(1-s)\left\|\mathbf{y}^{\prime}-a^{\prime} \mathbf{x}_{k}\right\|^{2}}{2 \sigma^{2}}}\right)^{\frac{1}{1-s}} \mathrm{~d} a^{\prime} \mathrm{d} \mathbf{y}^{\prime}} \\
& =\frac{\kappa_{s}(\mathbf{y}, a)}{\theta(s)} \frac{p_{A}(a)}{2 \pi \sigma^{2}} e^{-\frac{\left\|\mathbf{y}-a \mathbf{x}_{0}\right\|^{2}}{2 \sigma^{2}}}
\end{aligned}
$$

where the last equality follows from (B.2) and (B.3). The log-likelihood ratio $D_{0, s}$ in (A.11) is given by

$$
D_{0, s}(\mathbf{y}, a) \triangleq \ln \left(\frac{f_{s}(\mathbf{y}, a)}{P(\mathbf{y}, a \mid 0)}\right)=\ln \left(\kappa_{s}(\mathbf{y}, a)\right)-\ln (\theta(s))
$$

where the second equality follows from (120) and (B.4). The distribution $Q_{0, s}$ in (A.12) is given 
by

$$
\begin{aligned}
& Q_{0, s}(\mathbf{y}, a) \\
& \triangleq \frac{P(\mathbf{y}, a \mid 0)^{1-s} f_{s}(\mathbf{y}, a)^{s}}{\iint_{\mathbb{R}^{2}} \int_{0}^{\infty} P\left(\mathbf{y}^{\prime}, a^{\prime} \mid 0\right)^{1-s} f_{s}\left(\mathbf{y}^{\prime}, a^{\prime}\right)^{s} \mathrm{~d} a^{\prime} \mathrm{d} \mathbf{y}^{\prime}} \\
& =\frac{\frac{p_{A}(a)}{2 \pi \sigma^{2}} e^{-\frac{\left\|\mathbf{y}-a \mathbf{x}_{0}\right\|^{2}}{2 \sigma^{2}}}\left(\kappa_{s}(\mathbf{y}, a)\right)^{s}}{\iint_{\mathbb{R}^{2}} \int_{0}^{\infty} \frac{p_{A}\left(a^{\prime}\right)}{2 \pi \sigma^{2}} e^{-\frac{\left\|\mathbf{y}^{\prime}-a^{\prime} \mathbf{x}_{0}\right\|^{2}}{2 \sigma^{2}}}\left(\kappa_{s}\left(\mathbf{y}, a^{\prime}\right)\right)^{s} \mathrm{~d} a^{\prime} \mathrm{d} \mathbf{y}^{\prime}} \\
& \stackrel{(a)}{=} \frac{p_{A}(a)}{2 \pi \sigma^{2}} e^{-\frac{\left\|\mathbf{y}-a x_{0}\right\|^{2}}{2 \sigma^{2}}}\left(\kappa_{s}(\mathbf{y}, a)\right)^{s} \\
& \cdot\left[\int_{\mathbb{R}^{2}} \int_{0}^{\infty}\left(\frac{1}{M} \sum_{k=0}^{M-1}\left(\frac{1}{2 \pi \sigma^{2}} e^{-\frac{\left\|\mathbf{y}^{\prime}-a^{\prime} \mathbf{x}_{k}\right\|^{2}}{2 \sigma^{2}}}\right)^{1-s}\right)^{\frac{s}{1-s}}\left(\frac{p_{A}\left(a^{\prime}\right)}{2 \pi \sigma^{2}} e^{-\frac{\left\|\mathbf{y}^{\prime}-a^{\prime} \mathbf{x}_{0}\right\|^{2}}{2 \sigma^{2}}}\right)^{1-s} \mathrm{~d} a^{\prime} \mathrm{d} \mathbf{y}^{\prime}\right]^{-1} \\
& \underline{\underline{(b)}} \quad \frac{p_{A}(a)}{2 \pi \sigma^{2}} e^{-\frac{\left\|\mathbf{y}-a \mathbf{x}_{0}\right\|^{2}}{2 \sigma^{2}}}\left(\kappa_{s}(\mathbf{y}, a)\right)^{s} \\
& \iint_{\mathbb{R}^{2}} \int_{0}^{\infty}\left(\frac{1}{M} \sum_{k=0}^{M-1}\left(\frac{p_{A}\left(a^{\prime}\right)}{2 \pi \sigma^{2}} e^{-\frac{\left\|\mathbf{y}^{\prime}-a^{\prime} \mathbf{x}_{2}\right\|^{2}}{2 \sigma^{2}}}\right)^{1-s}\right)^{\frac{1}{1-s}} \mathrm{~d} a^{\prime} \mathrm{d} \mathbf{y}^{\prime} \\
& \stackrel{(c)}{=} \frac{\frac{p_{A}(a)}{2 \pi \sigma^{2}} e^{-\frac{\left\|\mathbf{y}-a \mathbf{x}_{0}\right\|^{2}}{2 \sigma^{2}}}\left(\kappa_{s}(\mathbf{y}, a)\right)^{s}}{\iint_{\mathbb{R}^{2}} \int_{0}^{\infty} \frac{p_{A}\left(a^{\prime}\right)}{2 \pi \sigma^{2}} e^{-\frac{\left\|\mathbf{y}^{\prime}-a^{\prime} x_{0}\right\|^{2}}{2 \sigma^{2}}} \kappa_{s}\left(\mathbf{y}, a^{\prime}\right) \mathrm{d} a^{\prime} \mathrm{d} \mathbf{y}^{\prime}} \\
& \stackrel{(d)}{=} \frac{p_{A}(a)\left(\kappa_{s}(\mathbf{y}, a)\right)^{s}}{2 \pi \sigma^{2} \theta(s)} e^{-\frac{\left\|\mathbf{y}-a \mathbf{x}_{0}\right\|^{2}}{2 \sigma^{2}}}
\end{aligned}
$$

where $(a)$ and $(c)$ rely on (B.2), (b) follows from Lemma 2.1 in the proof for symmetric memoryless channels, and $(d)$ relies on the definition of $\theta$ in (B.3). Substituting (B.5) and (B.6) in (A.10) we get

$$
\begin{aligned}
\mu_{0}^{\prime}(s) & =\mathbb{E}_{Q_{0, s}}\left(D_{0, s}\right) \\
& =\frac{1}{\theta(s)} \iint_{\mathbb{R}^{2}} \int_{0}^{\infty} \frac{p_{A}(a)}{2 \pi \sigma^{2}} e^{-\frac{\left\|\mathbf{y}-a \mathbf{x}_{0}\right\|^{2}}{2 \sigma^{2}}}\left(\kappa_{s}(\mathbf{y}, a)\right)^{s} \ln \left(\kappa_{s}(\mathbf{y}, a)\right) \mathrm{d} a \mathrm{~d} \mathbf{y}-\ln (\theta(s)) \\
\mu_{0}^{\prime \prime}(s) & =\mathbb{E}_{Q_{0, s}}\left(D_{0, s}^{2}(\mathbf{y})\right)-\mu_{0}^{\prime}(s)^{2} \\
& =\frac{1}{\theta(s)} \iint_{\mathbb{R}^{2}} \int_{0}^{\infty} \frac{p_{A}(a)}{2 \pi \sigma^{2}} e^{-\frac{\left\|\mathbf{y}-a \mathbf{x}_{0}\right\|^{2}}{2 \sigma^{2}}}\left(\kappa_{s}(\mathbf{y}, a)\right)^{s}\left(\ln \left(\kappa_{s}(\mathbf{y}, a)\right)-\ln (\theta(s))\right)^{2} \mathrm{~d} a \mathrm{~d} \mathbf{y}-\mu_{0}^{\prime}(s)^{2} .
\end{aligned}
$$

In this paper, we consider the particular case where the fading coefficients have a Rayleigh distribution. In this case, the distribution of the fading samples is given by $p_{A}(a)=2 a e^{-a^{2}}$ for $a \geq 0$, so that $\mathbb{E}\left(A^{2}\right)=1$. 


\section{B. M-ary PSK Modulated Signals over the AWGN Channel}

A widely studied special case of fully interleaved fading channels is the AWGN channel where the fading coefficients are set to 1 . Substituting $P_{A}(a)=\delta(a-1)$, where $\delta$ is the Dirac delta function at zero, we get that $\theta$ in (B.3) is particularized to

$$
\theta(s) \triangleq \iint_{\mathbb{R}^{2}} \frac{e^{-\frac{\left\|\mathbf{y}-\mathbf{x}_{0}\right\|^{2}}{2 \sigma^{2}}}}{2 \pi \sigma^{2}} \kappa_{s}(\mathbf{y}, a) \mathrm{d} \mathbf{y}
$$

and the first and second derivatives of $\mu_{0}$ with respect to $s$ (while holding $f_{s}$ fixed) are given by

$$
\begin{aligned}
& \mu_{0}^{\prime}(s)=\frac{1}{\theta(s)} \iint_{\mathbb{R}^{2}} \frac{e^{-\frac{\left\|\mathbf{y}-\mathbf{x}_{0}\right\|^{2}}{2 \sigma^{2}}}}{2 \pi \sigma^{2}}\left(\kappa_{s}(\mathbf{y}, a)\right)^{s} \ln \left(\kappa_{s}(\mathbf{y}, a)\right) \mathrm{d} \mathbf{y}-\ln (\theta(s)), \\
& \mu_{0}^{\prime \prime}(s)=\frac{1}{\theta(s)} \iint_{\mathbb{R}^{2}} \frac{1}{2 \pi \sigma^{2}} e^{-\frac{\left\|\mathbf{y}-\mathbf{x}_{0}\right\|^{2}}{2 \sigma^{2}}}\left(\kappa_{s}(\mathbf{y}, a)\right)^{s} \ln ^{2}\left(\frac{\kappa_{s}(\mathbf{y}, a)}{\theta(s)}\right) \mathrm{d} \mathbf{y}-\mu_{0}^{\prime}(s)^{2} .
\end{aligned}
$$

\section{The Binary Erasure Channel}

Let us denote the output of the channel when an erasure has occurred by $\mathcal{E}$, and let $p$ designate the erasure probability of the channel. Since the BEC is symmetric, the input distribution $\mathbf{q}_{s}$ which satisfies (67) is uniform (see Lemma A.1), and we get from (A.8)

$$
\mu_{0}\left(s, f_{s}\right)=(1-s) \ln \left(2(1-p)+2^{\frac{1}{1-s}} p\right)-\ln 2 .
$$

We now turn to calculate $f_{s}$ for the BEC; substituting the transition probabilities into (69) yields

$$
\begin{aligned}
& f_{s}(0)=f_{s}(1)=\frac{1-p}{2(1-p)+2^{\frac{1}{1-s}} p}, \\
& f_{s}(\mathcal{E})=\frac{2^{\frac{1}{1-s}} p}{2(1-p)+2^{\frac{1}{1-s}} p} .
\end{aligned}
$$

Substituting (B.13) and (B.14) into the definition of the distribution $Q_{0, s}$ in (A.12) gives

$$
\begin{aligned}
Q_{0, s}(0) & =\frac{P(0 \mid 0)^{1-s} f_{s}(0)^{s}}{\sum_{j \in\{0,1, \mathcal{E}\}} P(j \mid 0)^{1-s} f_{s}(j)^{s}}=\frac{1-p}{1-p+2^{\frac{s}{1-s}} p}, \\
Q_{0, s}(1) & =\frac{P(1 \mid 0)^{1-s} f_{s}(0)^{s}}{\sum_{j \in\{0,1, \mathcal{E}\}} P(j \mid 0)^{1-s} f_{s}(j)^{s}}=0 \\
Q_{0, s}(\mathcal{E}) & =\frac{P(\mathcal{E} \mid 0)^{1-s} f_{s}(\mathcal{E})^{s}}{\sum_{j \in\{0,1, \mathcal{E}\}} P(j \mid 0)^{1-s} f_{s}(j)^{s}}=\frac{2^{\frac{s}{1-s}} p}{1-p+2^{\frac{s}{1-s}} p}
\end{aligned}
$$


and the LLR in (A.11) is given by

$$
\begin{aligned}
& D_{0, s}(0)=\ln \left(\frac{1}{2(1-p)+2^{\frac{1}{1-s}} p}\right), \\
& D_{0, s}(\mathcal{E})=\ln \left(\frac{2^{\frac{1}{1-s}}}{2(1-p)+2^{\frac{1}{1-s}} p}\right) .
\end{aligned}
$$

Assembling (A.12), (B.15) and (B.16) yields

$$
\begin{aligned}
\mu_{0}^{\prime}\left(s, f_{s}\right) & =\mathbb{E}_{Q_{0, s}}\left(D_{0, s}\right) \\
& =\ln \left(\frac{1}{1-p+2^{\frac{s}{1-s}} p}\right)+\frac{2^{\frac{s}{1-s}} p}{1-p+2^{\frac{s}{1-s}} p} \frac{\ln 2}{1-s}, \\
\mu_{0}^{\prime \prime}\left(s, f_{s}\right) & =\mathbb{E}_{Q_{0, s}}\left(D_{0, s}^{2}\right)-\mu_{0}^{\prime}\left(s, f_{s}\right)^{2} \\
& =\frac{2^{\frac{s}{1-s}} p(1-p)}{\left(1-p+2^{\frac{s}{1-s}} p\right)^{2}}\left(\frac{\ln 2}{1-s}\right)^{2} .
\end{aligned}
$$

\section{APPENDIX C: PROOF OF PROPOSITION 4.2}

From the definition of $f_{N}$ in (95), it follows that

$$
\begin{aligned}
f_{N}(x) & =\frac{1}{2^{\frac{N-1}{2}} \Gamma\left(\frac{N+1}{2}\right)} \int_{0}^{\infty} z^{N-1} \exp \left(-\frac{z^{2}}{2}+z x\right) \mathrm{d} z \\
& =\frac{\exp \left(\frac{x^{2}}{2}\right)}{2^{\frac{N-1}{2}} \Gamma\left(\frac{N+1}{2}\right)} \int_{0}^{\infty} z^{N-1} \exp \left(-\frac{(z-x)^{2}}{2}\right) \mathrm{d} z \\
& =\frac{\exp \left(\frac{x^{2}}{2}\right)}{2^{\frac{N-1}{2}} \Gamma\left(\frac{N+1}{2}\right)} \int_{-x}^{\infty}(u+x)^{N-1} \exp \left(-\frac{u^{2}}{2}\right) \mathrm{d} u .
\end{aligned}
$$

From the binomial formula, we get

$$
f_{N}(x)=\frac{\exp \left(\frac{x^{2}}{2}\right)}{2^{\frac{N-1}{2}} \Gamma\left(\frac{N+1}{2}\right)} \sum_{j=0}^{N-1}\left[\left(\begin{array}{c}
N-1 \\
j
\end{array}\right) x^{N-1-j} \int_{-x}^{\infty} u^{j} \exp \left(-\frac{u^{2}}{2}\right) \mathrm{d} u\right] .
$$

We now examine the integrals in the right side of (C.1). For odd values of $j$, we get

$$
\begin{aligned}
\int_{-x}^{\infty} u^{j} \exp \left(-\frac{u^{2}}{2}\right) \mathrm{d} u & =\int_{-x}^{x} u^{j} \exp \left(-\frac{u^{2}}{2}\right) \mathrm{d} u+\int_{x}^{\infty} u^{j} \exp \left(-\frac{u^{2}}{2}\right) \mathrm{d} u \\
& =\int_{x}^{\infty} u^{j} \exp \left(-\frac{u^{2}}{2}\right) \mathrm{d} u \\
& =\int_{0}^{\infty} u^{j} \exp \left(-\frac{u^{2}}{2}\right) \mathrm{d} u-\int_{0}^{x} u^{j} \exp \left(-\frac{u^{2}}{2}\right) \mathrm{d} u
\end{aligned}
$$

where the second equality follows since the integrand is an odd function for odd values of $j$, and the interval of first integral is symmetric around zero (so this integral vanishes). For even 
values of $j$, we get

$$
\begin{aligned}
\int_{-x}^{\infty} u^{j} \exp \left(-\frac{u^{2}}{2}\right) \mathrm{d} u & =\int_{0}^{\infty} u^{j} \exp \left(-\frac{u^{2}}{2}\right) \mathrm{d} u+\int_{-x}^{0} u^{j} \exp \left(-\frac{u^{2}}{2}\right) \mathrm{d} u \\
& =\int_{0}^{\infty} u^{j} \exp \left(-\frac{u^{2}}{2}\right) \mathrm{d} u+\int_{0}^{x} u^{j} \exp \left(-\frac{u^{2}}{2}\right) \mathrm{d} u
\end{aligned}
$$

where the second equality holds since the integrand is an even function for even values of $j$. Combining (C.2) and (C.3) gives that for $j \in\{0,1, \ldots, N-1\}$

$$
\begin{aligned}
\int_{-x}^{\infty} u^{j} \exp \left(-\frac{u^{2}}{2}\right) \mathrm{d} u & =\int_{0}^{\infty} u^{j} \exp \left(-\frac{u^{2}}{2}\right) \mathrm{d} u+(-1)^{j} \int_{0}^{x} u^{j} \exp \left(-\frac{u^{2}}{2}\right) \mathrm{d} u \\
& \stackrel{(a)}{=} \int_{0}^{\infty}(2 t)^{\frac{j-1}{2}} \exp (-t) \mathrm{d} t+(-1)^{j} \int_{0}^{\frac{x^{2}}{2}}(2 t)^{\frac{j-1}{2}} \exp (-t) \mathrm{d} t \\
& =2^{\frac{j-1}{2}} \int_{0}^{\infty} t^{\frac{j-1}{2}} \exp (-t) \mathrm{d} t\left[1+(-1)^{j} \frac{\int_{0}^{\frac{x^{2}}{2}} t^{\frac{j-1}{2}} \exp (-t) \mathrm{d} t}{\int_{0}^{\infty} t^{\frac{j-1}{2}} \exp (-t) \mathrm{d} t}\right] \\
& =2^{\frac{j-1}{2}} \Gamma\left(\frac{j+1}{2}\right)\left[1+(-1)^{j} \tilde{\gamma}\left(\frac{x^{2}}{2}, \frac{j+1}{2}\right)\right]
\end{aligned}
$$

where $(a)$ follows by substituting $t \triangleq \frac{u^{2}}{2}$ and the functions $\Gamma$ and $\tilde{\gamma}$ are introduced in (112) and (113), respectively. Substituting the last equality in (C.1) and also noting that

$$
\left(\begin{array}{c}
N-1 \\
j
\end{array}\right)=\frac{\Gamma(N)}{\Gamma(N-j) \Gamma(j+1)}, \quad N \in \mathbb{N}, j \in\{0,1, \ldots, N-1\}
$$

we get

$$
\begin{aligned}
f_{N}(x)= & \frac{\exp \left(\frac{x^{2}}{2}\right)}{2^{\frac{N-1}{2}} \Gamma\left(\frac{N+1}{2}\right)} \sum_{j=0}^{N-1}\left\{\frac{\Gamma(N)}{\Gamma(N-j) \Gamma(j+1)} x^{N-1-j} 2^{\frac{j-1}{2}}\right. \\
& \left.\cdot \Gamma\left(\frac{j+1}{2}\right)\left[1+(-1)^{j} \tilde{\gamma}\left(\frac{x^{2}}{2}, \frac{j+1}{2}\right)\right]\right\} \\
= & \sum_{j=0}^{N-1}\left\{\frac{\exp \left(\frac{x^{2}}{2}\right)}{\Gamma(N-j)} \frac{\Gamma(N)}{\Gamma\left(\frac{N+1}{2}\right)} \frac{\Gamma\left(\frac{j+1}{2}\right)}{\Gamma(j+1)} \frac{x^{N-1-j}}{2^{\frac{N-j}{2}}}\left[1+(-1)^{j} \tilde{\gamma}\left(\frac{x^{2}}{2}, \frac{j+1}{2}\right)\right]\right\} \\
& \stackrel{(a)}{=} \sum_{j=0}^{N-1}\left\{\frac{\exp \left(\frac{x^{2}}{2}\right)}{\Gamma(N-j)} \frac{2^{N-1} \Gamma\left(\frac{N}{2}\right)}{\sqrt{\pi}} \frac{2^{-j} \sqrt{\pi}}{\Gamma\left(\frac{j}{2}+1\right)} \frac{x^{N-1-j}}{2^{\frac{N-j}{2}}}\left[1+(-1)^{j} \tilde{\gamma}\left(\frac{x^{2}}{2}, \frac{j+1}{2}\right)\right]\right\} \\
& \stackrel{(b)}{=} \sum_{j=0}^{N-1} \exp (d(N, j, x))
\end{aligned}
$$

where $(a)$ follows from the equality

$$
\Gamma(2 u)=\frac{2^{2 u-1}}{\sqrt{\pi}} \Gamma(u) \Gamma\left(u+\frac{1}{2}\right), \quad u \neq 0,-\frac{1}{2},-1,-\frac{3}{2}, \ldots
$$

and $(b)$ follows from the definition of the function $d$ in (111). 


\section{REFERENCES}

[1] B. Ammar, Y. Kou, J. Xu and S. Lin, "Construction of low-density parity-check codes based on balanced incomplete block designs," IEEE Trans. on Information Theory, vol. 50, no. 6, pp. 1257-1268, June 2004.

[2] E. R. Berlekamp, "The performance of block codes," Notices of the AMS, pp. 17-22, January 2002. [Online]. Available: http://www.ams.org/notices/200201/fea-berlekamp.pdf.

[3] D. J. Costello and G. D. Forney, "Channel coding: The road to channel capacity," Proceedings of the IEEE, vol. 95, no. 6, pp. 1150-1177, June 2007.

[4] J. Cuevas, P. Adde and S. Kerouedan, "Turbo decoding of product codes for Gigabit per second applications and beyond," European Transactions on Telecommunications, vol. 17, no. 1, pp. 45-55, Jan.-Feb. 2006.

[5] C. Di, D. Proietti, I. E. Telatar and R. Urbanke, "Finite-length analysis of low-density parity-check codes," IEEE Trans. on Information Theory, vol. 48, no. 6, pp. 1570-1579, June 2002.

[6] D. Divsalar and S. Dolinar, "Concatenation of Hamming codes and accumulator codes with high-order modulations for high-speed decoding," Jet Propulsion Laboratory, IPN Progress Report 42-156, February 15, 2004.

[7] D. Divsalar and C. Jones, "Protograph LDPC codes with node degrees at least 3," Proceedings of the 2006 IEEE Global Communications Conference, San Francisco, CA, USA, 27 November-1 December 2006.

[8] D. Divsalar, C. Jones, S. Dolinar and J. Thorpe, "Protograph based LDPC codes with minimum distance linearly growing with block size," Proceedings of the 2005 IEEE Global Communications Conference, vol. 3 , pp. 1152-1156, St. Louis, MO, USA, 28 November-2 December 2005.

[9] S. Dolinar, D. Divsalar and F. Pollara, “Code performance as a function of block size," Jet Propulsion Laboratory (JPL), TMO Progress Report 42-133, May 15, 1998.

[10] P. Elias, "List decoding for noisy channels," MIT Res. Lab. Electron., Cambridge, MA, USA, September 1957.

[11] R. G. Gallager, "A simple derivation of the coding theorem and some applications," IEEE Trans. on Information Theory, vol. 11, pp. 3-18, January 1965.

[12] R. G. Gallager, Information Theory and Reliable Communications, John Wiley, 1968.

[13] V. Guruswami, "Algorithmic Results in List Decoding," Foundations and Trends in Theoretical Computer Science, Now Publishers, Delft, the Netherlands, vol. 2, no. 2, pp. 107-195, 2006.

[14] H. Herzberg and G. Poltyrev, "The error probability of M-ary PSK block coded modulation schemes," IEEE Trans. on Communications, vol. 44, no. 4, pp. 427-433, April 1996.

[15] D. E. Lazic, Th. Beth and M. Calic, "How close are turbo codes to optimal codes ?," Proceedings of the International Symposium on Turbo Codes and Related Topics, pp. 192-195, Brest, France, 3-5 September 1997.

[16] D. E. Lazic, Th. Beth and S. Egner, "Constrained capacity of the AWGN channel," IEEE 1998 International Symposium on Information Theory (ISIT 1998), p. 237, Cambridge, MA, USA, 16-21 August, 1998.

[17] M. G. Luby, M. Mitzenmacher, M. A. Shokrollahi and D. A. Spielman, "Efficient erasure correcting codes," IEEE Trans. on Information Theory, vol. 47, no. 2, pp. 569-584, February 2001.

[18] S. J. Macmullan and O. M. Collins, "A comparison of known codes, random codes and the best codes," IEEE Trans. on Information Theory, vol. 44, no. 7, pp. 3009-3022, November 1998.

[19] H. Pfister and I. Sason, "Accumulate-repeat-accumulate codes: Capacity-achieving ensembles of systematic codes for the erasure channel with bounded complexity," IEEE Trans. on Information Theory, vol. 53, no. 6, pp. 2088-2115, June 2007.

[20] G. Poltyrev, "Bounds on the decoding error probability of binary linear codes via their spectra," IEEE Trans. on Information Theory, vol. 40, no. 4, pp. 1284-1292, July 1994.

[21] T. Richardson and R. Urbanke, Modern Coding Theory, Cambridge University Press, 2008.

[22] R. M. Roth, Introduction to Coding Theory, Cambridge University Press, 2006.

[23] I. Sason and S. Shamai, "On improved bounds on the decoding error probability of block codes over interleaved fading channels, with applications to turbo-like codes," IEEE Trans. on Information Theory, vol. 47, no. 6, pp. 2275-2299, September 2001.

[24] I. Sason and S. Shamai, "Performance Analysis of Linear Codes under Maximum-Likelihood Decoding: A Tutorial," Foundations and Trends in Communications and Information Theory, vol. 3, no. 1-2, pp. 1-222, Now Publishers, Delft, the Netherlands, July 2006. [Online]. Available: http://webee.technion.ac.il/people/sason/ monograph_postprint.pdf.

[25] C. E. Shannon, "Probability of error for optimal codes in a Gaussian channel," Bell System Technical Journal, vol. 38, pp. 611-656, May 1959. 
[26] C. Shannon, R. Gallager and E. Berlekamp, "Lower bounds to error probability for decoding on discrete memoryless channels," Information and Control, vol. 10, Part 1: pp. 65-103, and Part 2: pp. 522-552, February/May 1967.

[27] Claude Elwood Shannon - Collected Papers, edited by N. J. A. Sloane and A. D. Wyner, IEEE Press, 1993.

[28] A. Shokrollahi, "New sequences of time erasure codes approaching channel capacity," in Proceedings of the 13th International Symposium on Applied Algebra, Algebraic Algorithms and Error-Correcting Codes, Lectures Notes in Computer Science 1719, Springer Verlag, pp. 65-76, 1999.

[29] Y. Tai, L. Lan, L. Zeng, S. Lin and K. Abdel-Ghaffar, "Algebraic construction of quasi-cyclic LDPC codes for the AWGN and erasure channels," IEEE Trans. on Communications, vol. 54, no. 10, pp. 1756-1765, October 2006.

[30] O. Y. Takeshita, O. M. Collins, P. C. Massey and D. J. Costello, "On the frame-error rate of concatenated turbo codes," IEEE Trans. on Communications, vol. 49, no. 4, pp. 602-608, April 2001.

[31] H. Tang, J. Xu, S. Lin and K. Abdel-Ghaffar, "Codes on finite geometries," IEEE Trans. on Information Theory, vol. 51, no. 2, pp. 572-596, February 2005.

[32] M. Twitto and I. Sason, "On the error exponents of some improved tangential-sphere bounds," IEEE Trans. on Information Theory, vol. 53, no. 3, pp. 1196-1210, March 2007.

[33] M. Twitto, I. Sason and S. Shamai, "Tightened upper bounds on the ML decoding error probability of binary linear block codes," IEEE Trans. on Information Theory, vol. 53, no. 4, pp. 1495-1510, April 2007.

[34] R. Urbanke, Error floor calculator for the binary erasure channel. [Online]. Available: http://lthcwww.epfl.ch/ research/efc/.

[35] A. Valembois and M. Fossorier, "Box and match techniques applied to soft-decision decoding," IEEE Trans. on Information Theory, vol. 50, no. 5, pp. 796-810, May 2004.

[36] A. Valembois and M. Fossorier, "Sphere-packing bounds revisited for moderate block length," IEEE Trans. on Information Theory, vol. 50, no. 12, pp. 2998-3014, December 2004.

[37] C. C. Wang, S. R. Kulkarni and H. V. Poor, "Finite-dimensional bounds on $\mathbb{Z}_{m}$ and binary LDPC codes with belief-propagation decoders," IEEE Trans. on Information Theory, vol. 53, no. 1, pp. 56-81, January 2007.

[38] L. Wei, "Near-optimum serial concatenation of single-parity codes with convolutional codes," IEE Proceedings on Communications, vol. 152, no. 4, pp. 397-403, August 2005.

[39] J. M. Wozencraft, "List decoding," Quarterly Progress Report, MIT Research Laboratory of Electronics, vol. 48, pp. 90-95, January 1958. 